\title{
Reinvestigation of a Catalytic, Enantioselective Alkene Dibromination and Chlorohydroxylation
}

Scott E. Denmark* and Nessa Carson

Roger Adams Laboratory, University of Illinois, 600 South Mathews Avenue, Urbana, Illinois 61801

\section{SUPPORTING INFORMATION}

TABLE OF CONTENTS

Henry's Dibromination Results

General Experimental

Literature Preparations

Experimental Procedures

Preparation and Isolation of Palladium Catalyst 3a

Henry's Preparation of $\mu$-(R)-BINAP $\mu$-1-Phenylhexane-1,3,5-triketone

Bis[(acetonitrile)palladium(II)] Tetrafluoroborate 4a

New Preparation of Dinuclear Palladium Catalysts 4

Preparation of $\mu$ - $(R)$-BINAP $\mu$-1-Phenylhexane-1,3,5-trione

Bis[(acetonitrile)palladium(II)] Tetrafluoroborate 4a

Preparation of $\mu$ - $(R)$-Tol-BINAP $\mu$-1-Phenylhexane-1,3,5-trione

Bis[(acetonitrile)palladium(II)] Tetrafluoroborate $\mathbf{4 b}$

Preparation of Racemic Dibromide Standards

Preparation of Racemic Standards 6a, $\mathbf{6 b}$, and $\mathbf{6 d}$

Preparation of Racemic Standard 6c

Conditions and Spectra for Dibromination Reactions

Conditions and Spectra for Chlorohydroxylation Reactions

Chiral Shift Reagent NMR Experiment

PAGE

S3

S4

S5

S7

S8

S11

S11

S15

S21

S36

S45

$S 45$

References

NMR Spectra

Allyl 4-Methoxyphenyl Ether 5a

546

Allyl 4-Cyanophenyl Ether 5b

548

Allyl Phenyl Ether 5c

S50

Allyl 2,6-Diisopropylphenyl Ether 5d

S52

Methyl Vinyl Ketone (commercial, purified)

S54

Allyl 1-Naphthyl Ether 1c

S56

Racemic 1-(2,3-Dibromopropoxy)-4-methoxybenzene 6a 
Racemic 1-(2,3-Dibromopropoxy)-4-cyanobenzene 6b $\quad S 60$

Racemic (2,3-Dibromopropoxy)benzene 6c S62

Racemic 1-(2,3)-Dibromopropoxy)-2,6-diisopropylbenzene 6d $\quad S 64$

Racemic (3-Chloro-2-hydroxypropoxy)benzene 2a $\quad S 66$

Racemic 1-(3-Chloro-2-hydroxypropoxy)naphthalene 2b $\quad S 68$

Racemic 4-Chloro-3-hydroxybutan-2-one 2c $\quad S 70$

(R)-BINAP bis(acetonitrile)palladium(II) tetrafluoroborate 3a $\quad S 72$

1-Phenylhexane-1,3,5-trione $\quad S 74$

$\mu$-(R)-BINAP $\mu$-1-phenylhexane-1,3,5-trione bis[(acetonitrile)palladium(II)] $\quad S 76$

tetrafluoroborate 4a $\quad S 76$

$\mu$ - $(R)$-Tol-BINAP $\mu$-1-phenylhexane-1,3,5-trione bis[(acetonitrile)palladium(II)]
tetrafluoroborate $\mathbf{4 b}$

Chiral Shift Reagent NMR Experiments

Crude Reaction Mixtures

Run 1 Crude 5a Dibromination Mixture

Run 2 Crude 5a Dibromination Mixture

Run 3 Crude 5a Dibromination Mixture

Run 4 Crude 5a Dibromination Mixture

Run 5 Crude 5b Dibromination Mixture

Run 6 Crude 5c Dibromination Mixture

Run 7 Crude 5d Dibromination Mixture

Run 8 Crude 2a Chlorohydroxylation Mixture

Run 9 Crude 2a Chlorohydroxylation Mixture

Run 10 Crude 2b Chlorohydroxylation Mixture

Run 11 Crude 2c Chlorohydroxylation Mixture 


\section{Henry's Dibromination Results ${ }^{1}$}

Table S1. Henry's Reported Asymmetric Dibromination Results

\begin{tabular}{|c|c|c|c|c|c|c|}
\hline Entry & Product $^{\mathrm{a}}$ & $\begin{array}{c}\text { Nuclearity of } \\
\text { catalyst }\end{array}$ & Ligand & $\begin{array}{c}\text { Recovered } \\
\text { starting } \\
\text { material }\end{array}$ & $\begin{array}{l}\text { Dibromide } \\
\text { yield, \% }{ }^{\mathrm{b}}\end{array}$ & $\mathrm{er}^{\mathrm{c}}$ \\
\hline 1 & & $\begin{array}{c}\text { Ambiguous in } \\
\text { original text }\end{array}$ & $(S)$-BINAP & NR & 95 & $98: 2$ \\
\hline 2 & & 2 & $(S)$-Tol-BINAP & NR & 95 & $98.5: 1.5$ \\
\hline 3 & & 2 & $(S)$-BINAP & NR & 95 & $97.5: 2.5$ \\
\hline 4 & & 1 & $(S)$-BINAP & NR & 95 & $97: 3$ \\
\hline 5 & & 2 & (S)-METBOX & $63 \%$ & 31 & $97: 3$ \\
\hline 6 & & 1 & $(S)-\mathrm{BZOX}$ & $30 \%$ & $80^{\mathrm{d}}$ & $92: 8$ \\
\hline 7 & & 2 & $(S)$-METBOX & $21 \%$ & 70 & $91: 9$ \\
\hline 8 & $\mathrm{Br}$ & 2 & $(R)$-BINAM & $24 \%$ & 77 & $90: 10$ \\
\hline 9 & & 2 & $(R)$-BINAM & $12 \%$ & 83 & $57: 43$ \\
\hline 10 & $B_{r}$ & 2 & $(S)$-Tol-BINAP & NR & $85^{\mathrm{e}}$ & - \\
\hline
\end{tabular}

$\mathrm{NR}=$ not reported. ${ }^{\mathrm{a}}$ Absolute configuration not determined. ${ }^{\mathrm{b}}$ Isolated yield. Yields differ from the values in the original paper since they are calculated from the amount of olefin substrate used rather than the amount that was converted under the reaction conditions, where this information is available. ${ }^{\mathrm{c}} \mathrm{er}$ of dibromide products determined by ${ }^{1} \mathrm{H}$ chiral shift reagent NMR. ${ }^{\mathrm{d}}$ Yield calculated from oxygen uptake. ${ }^{\mathrm{e}}$ An unnamed byproduct was formed in approximately $14 \%$ yield by GC analysis. 


\section{General Experimental}

Reaction Setup: Reactions were performed in oven-dried glassware. Room temperature (rt) was approximately $23{ }^{\circ} \mathrm{C}$. Solvent evaporation was performed on a rotary evaporator at $30{ }^{\circ} \mathrm{C}$ unless otherwise specified.

NMR Spectroscopy: Spectra were recorded on Varian $500 \mathrm{MHz}$ spectrometers. ${ }^{1} \mathrm{H}$ and ${ }^{13} \mathrm{C}$ spectra were referenced to residual non-deuterated chloroform $\left(7.26 \mathrm{ppm},{ }^{1} \mathrm{H} ; 77.02 \mathrm{ppm},{ }^{13} \mathrm{C}\right)$ or $d_{6^{-}}$ DMSO

(2.49 ppm, $\left.{ }^{1} \mathrm{H} ; 39.52 \mathrm{ppm},{ }^{13} \mathrm{C}\right) .{ }^{31} \mathrm{P}$ spectra were referenced to an external standard of $85 \% \mathrm{H}_{3} \mathrm{PO}_{4}$ in $\mathrm{H}_{2} \mathrm{O}$ $(0 \mathrm{ppm}) .{ }^{13} \mathrm{C}$ spectra were recorded with broadband decoupling of the ${ }^{1} \mathrm{H}$ nucleus, and ${ }^{31} \mathrm{P}$ spectra with broadband decoupling of the ${ }^{13} \mathrm{C}$ nucleus. Chemical shifts are reported in parts per million (ppm), multiplicities indicated by s (singlet), d (doublet), t (triplet), sext (sextet), sept (septet), br (broad), and $\mathrm{m}$ (multiplet). Coupling constants, $J$, are reported in $\mathrm{Hz}$ with integration provided and assignments indicated.

Infrared Spectroscopy: Infrared spectra (IR) were recorded using a Perkin-Elmer FTIR instrument. Peaks are reported in $\mathrm{cm}^{-1}$ with indicated relative absorption intensities: s (strong, 67-100\%); m (medium, 34-66\%); w (weak, 0-33\%) and sh (sharp) and br (broad) assignments.

Mass Spectrometry: Matrix-assisted laser desorption/ionization (MALDI) spectrometry was performed on an AB Voyager instrument. Electron Impact (EI) spectrometry was performed at $70 \mathrm{eV}$ using methane as the carrier gas, with a time-of-flight (TOF) mass analyzer. Data are reported in the form of $\mathrm{m} / \mathrm{z}$ (intensity relative to the base peak $=100$ ).

Distillation: Bulb-to-bulb distillation was performed using a Kugelrohr, with boiling points (bp) corresponding to uncorrected air bath temperatures (ABT) under vacuum.

Gas Chromatography: Analytical gas chromatography (GC) was performed using a flame ionization detector and an Agilent CycloSil-B column. Injector temperature was $250{ }^{\circ} \mathrm{C}$, and detector temperature was $300^{\circ} \mathrm{C}$, with a $\mathrm{H}_{2}$ carrier gas flow of $4.7 \mathrm{~mL} \mathrm{~min}^{-1}$.

Liquid Chromatography: Analytical thin-layer chromatography (TLC) was performed on silica gel $60 \mathrm{~F}_{254}$ plates. Retention factor $\left(\mathrm{R}_{\mathrm{f}}\right)$ values reported were measured using $10 \times 2 \mathrm{~cm}$ silica TLC plates in a developing chamber containing the solvent system described. Visualization was accomplished with UV light and cerium(IV) ammonium molybdate solution (CAM), acidic 2,4-dinitrophenylhydrazine solution (DNP), or a solution of silver nitrate and 2-phenoxyethanol with one drop of 30\% aqueous hydrogen peroxide and UV irradiation for several minutes $\left(\mathrm{AgNO}_{3}-\mathrm{H}_{2} \mathrm{O}_{2}\right)$. Flash column chromatography 
was performed using 40-63 $\mu \mathrm{m}$ particle size (230-400 mesh, $60 \AA$ pore size) $\mathrm{SiO}_{2}$. Analytical highperformance liquid chromatography (HPLC) was performed on an Agilent 1100 system using a UV detector $(254 \mathrm{~nm})$ and a Chiralpak IB-3 or OJ-H column. Analytical supercritical fluid chromatography (SFC) was performed on an Agilent 1100 HPLC equipped with an Aurora Systems A-5 supercritical $\mathrm{CO}_{2}$ adapter for SFC, using a UV detector $(220 \mathrm{~nm})$ and a Daicel Chiralcel OD column.

Solvents: Anhydrous toluene (ACS grade), anhydrous benzene, anhydrous diethyl ether (ACS grade, BHT-stabilized), anhydrous 1,2-dimethoxyethane (DME), and anhydrous tetrahydrofuran (THF, HPLC grade) were dried by percolation through two columns packed with neutral alumina, under a positive pressure of argon. Anhydrous acetonitrile was freshly distilled from sodium. Anhydrous acetone was prepared by stirring acetone (Optima grade) with $10 \mathrm{~g} \mathrm{~L}^{-1}$ anhydrous calcium sulfate for $4 \mathrm{~h}$ under Ar, followed by distillation from fresh anhydrous calcium sulfate. ${ }^{2}$ Other solvents for reaction, filtration, transfers and chromatography were hexane (Optima grade), toluene (ACS grade), dichloromethane (ACS grade), chloroform (ACS grade), ethyl acetate (ACS grade), acetone (Optima grade), 95\% or 100\% ethanol (USP grade), and methanol (Optima grade). Degassing of solvents was achieved by sparging with Ar.

Chemicals: Copper(II) bromide, lithium bromide, sodium hydride and tetrakis(acetonitrile)palladium(II) tetrafluoroborate were stored in a glove box under $\operatorname{Ar}$. $(R)$-BINAP and $(R)$-Tol-BINAP were purified by basic aqueous extraction from toluene, trituration with degassed $\mathrm{MeOH}$ and subsequent recrystallization from 1:1 toluene:EtOH. ${ }^{2}$ Anhydrous triethylamine was freshly distilled from calcium hydride. All other reagents were used as received.

\section{Literature Preparations}

Allyl aryl ethers $\mathbf{1 c}$ and $\mathbf{5 a - 5 d}$ were prepared according to a literature procedure, ${ }^{3,4}$ were purified via Kugelrohr distillation or sublimation, and had spectroscopic data consistent with literature.

(R)-BINAP bis(acetonitrile)palladium(II) tetrafluoroborate $3 \mathbf{b}^{5}$ (highly air-sensitive) and 1phenylhexane-1,3,5-trione ${ }^{6}$ were also prepared according to literature procedures, and had spectroscopic data consistent with literature. 


\section{Experimental Procedures}

\section{Preparation and Isolation of Palladium Catalyst 3aa}
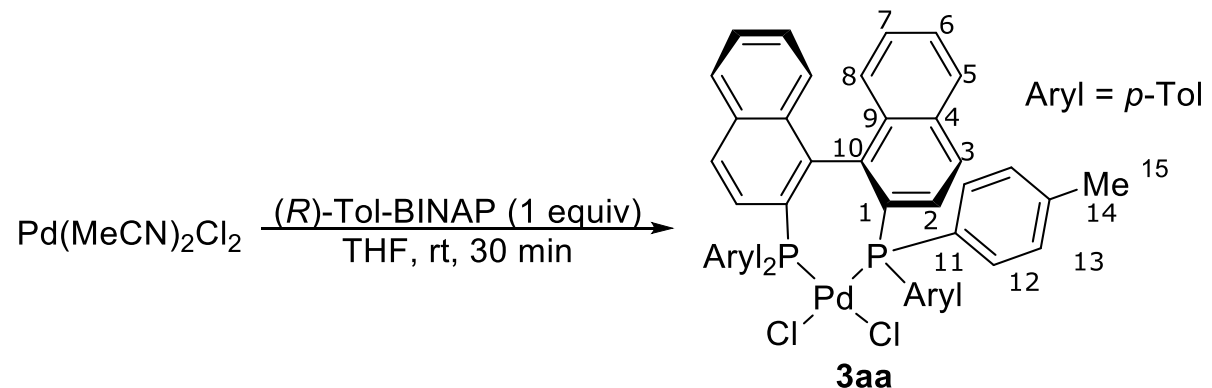

Complex 3aa was generated in situ for Table 1, Entries 8 and 10, and a sample was isolated for characterization purposes.

Bis(acetonitrile)dichloropalladium(II) $(20 \mathrm{mg}, 77 \mu \mathrm{mol})$ was charged to a $10-\mathrm{mL}$, two-necked, round-bottomed flask, in a glove box. Anhydrous, degassed THF $(0.5 \mathrm{~mL})$ was added, followed by $(R)-$ Tol-BINAP (51 mg, $77 \mu \mathrm{mol}, 1.0$ equiv), washing with further anhydrous, degassed THF $(0.5 \mathrm{~mL})$. The clear, orange-yellow solution was stirred at $\mathrm{rt}$ for $30 \mathrm{~min}$. Anhydrous, degassed $\mathrm{Et}_{2} \mathrm{O}(3 \mathrm{~mL})$ was added, and a yellow precipitate was immediately produced. The solution was filtered, and the filtrand dried in vacuo to $62 \mathrm{mg}$ ( $77 \mu \mathrm{mol}, \mathbf{9 7 \%}$ yield) of a bright yellow powder. Further drying under high vacuum (50 $\mathrm{Pa}$ ) for over $24 \mathrm{~h}$ did not remove residual $\mathrm{Et}_{2} \mathrm{O}$.

\section{Data for 3b:}

${ }^{1} \mathrm{H}$ NMR: $\quad\left(500 \mathrm{MHz}, d_{6}\right.$-DMSO)

$\delta 7.73(\mathrm{~d}, J=8.9 \mathrm{~Hz}, 2 \mathrm{H}), 7.70(\mathrm{~d}, J=8.2 \mathrm{~Hz}, 2 \mathrm{H}), 7.61(\mathrm{dd}, J=11.7,8.2 \mathrm{~Hz}, 4 \mathrm{H}), 7.50(\mathrm{t}, J$

$=7.5 \mathrm{~Hz}, 2 \mathrm{H}), 7.39$ (br s, $4 \mathrm{H}), 7.33(\mathrm{~d}, J=7.4 \mathrm{~Hz}, 4 \mathrm{H}), 7.14-7.24(\mathrm{~m}, 4 \mathrm{H}), 6.63$ (d, $J=8.7$

$\mathrm{Hz}, 2 \mathrm{H}$ ), 6.53 (br s, $4 \mathrm{H}$ ), 2.38 (s, $\left.6 \mathrm{H}, \mathrm{H}_{3, \mathrm{~A}} \mathrm{C}(15)\right), 1.98$ (s, $6 \mathrm{H}, \mathrm{H}_{3, \mathrm{~B}} \mathrm{C}(15)$ )

${ }^{31}$ P NMR: $\quad\left(202 \mathrm{MHz}, d_{6}\right.$-DMSO)

28.62

IR: $(\mathrm{KBr} ;$ pressed under $\mathrm{Ar})$

3416 (s, v br), 3055 (s), 2976 (s), 2920 (s), 2866 (s), 1597 (s), 1557 (m), 1498 (s), 1444 (m), 1397 (m), 1308 (m), 1224 (w), 1192 (m), 1159 (w), 1101 (s), 1020 (w), 870 (w), 846 (w), 803 (s), $746(\mathrm{~s}), 707(\mathrm{~m}), 697(\mathrm{~m}), 670(\mathrm{~m}), 652(\mathrm{~m}), 636(\mathrm{~m}), 621(\mathrm{~m}), 614(\mathrm{~m}), 602(\mathrm{~m}), 530(\mathrm{~m})$, $523(\mathrm{~m}), 508(\mathrm{~s}), 475(\mathrm{~m}), 454(\mathrm{~m})$

MS: (MALDI; THF)

$819.96\left([\mathrm{M}-\mathrm{Cl}]^{+}\right)$ 


\section{Henry's Preparation of $\mu$-(R)-BINAP $\mu$-1-Phenylhexane-1,3,5-triketone Bis[(acetonitrile)palladium(II)] Tetrafluoroborate 4a}

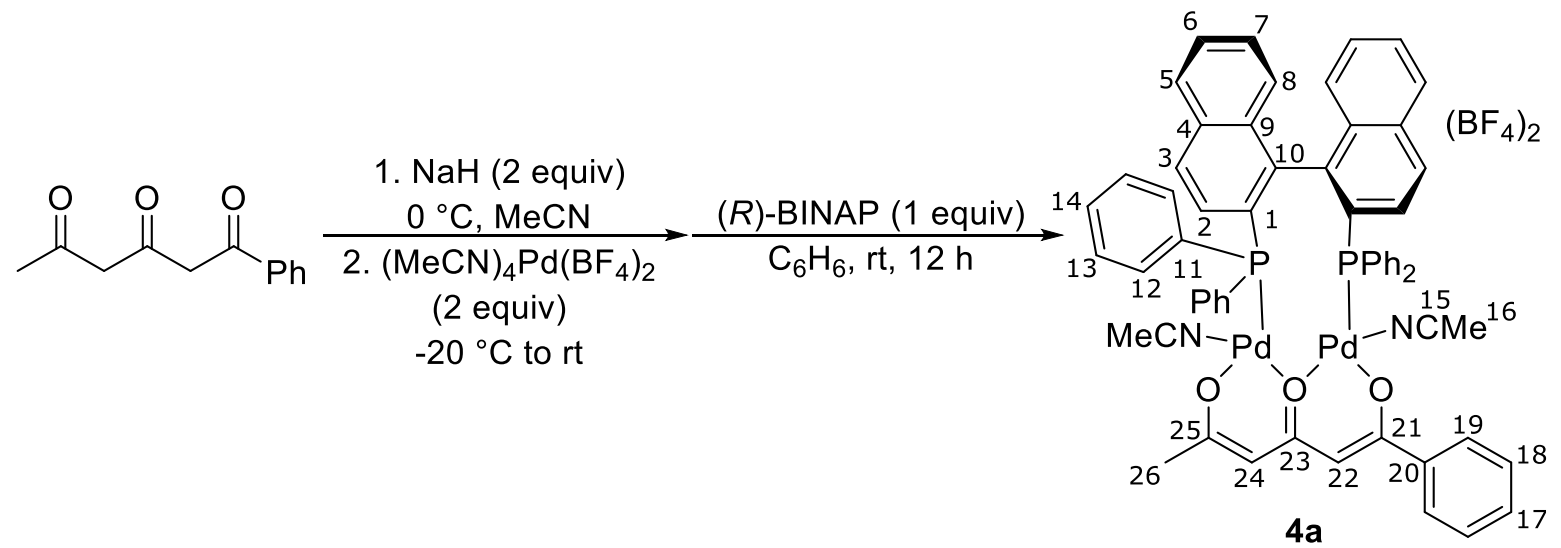

The procedure was followed as closely as possible to that in Henry's report. ${ }^{1}$

To a stirred so lution of tetrakis(acetonitrile)palladium(II) tetrafluoroborate (100 mg, $226 \mu$ mol, 2 equiv) in anhydrous acetonitrile $(4.3 \mathrm{~mL})$ under $\mathrm{Ar}$ in a $25-\mathrm{mL}$, round-bottomed flask was added, dropwise, a solution of 1-phenylhexane-1,3,5-trione $(23 \mathrm{mg}, 113 \mu \mathrm{mol})$ in anhydrous acetonitrile $(2.0 \mathrm{~mL})$. Anhydrous triethylamine ( $210 \mu \mathrm{L}, 152 \mathrm{mg}, 13.4$ equiv) was then added dropwise, causing a color change from yellow to bold orange. The solution was stirred at rt for $30 \mathrm{~min}$. A solution of (R)-BINAP (70 mg, $113 \mu \mathrm{mol}, 1$ equiv) in anhydrous benzene $(1.3 \mathrm{~mL})$ was then added dropwise to the reaction mixture, which was next stirred at rt for $12 \mathrm{~h}$. The mixture was concentrated under high vacuum (50 Pa) to a pasty, brick red residue. Anhydrous $\mathrm{Et}_{2} \mathrm{O}(10 \mathrm{~mL})$ was added, and the suspension stirred at high frequency for $15 \mathrm{~min}$. Solvent was removed under high vacuum to yield a brick red powder. This was washed in a glove box with anhydrous $\mathrm{Et}_{2} \mathrm{O}(5 \times 30 \mathrm{~mL})$ to give a yellow-brown powder, which was dried briefly in vacuo to $131 \mathrm{mg}$ yellow-brown powder $\left(100 \mu \mathrm{mol}, \mathbf{9 0 \%}\right.$ yield. Residual $\mathrm{Et}_{2} \mathrm{O}$ was not removed by further drying a sample in vacuo for over $24 \mathrm{~h}$, and the ${ }^{31} \mathrm{P}$ NMR spectrum did not change after this process.

\section{${ }^{1} \mathrm{H}$ NMR: $\quad\left(500 \mathrm{MHz}, d_{6}\right.$-DMSO)}

$\delta$ 6.95-8.19 (m, $12.09 \mathrm{H}), 6.83(\mathrm{~m}, 0.41 \mathrm{H}), 6.60(\mathrm{~m}, 0.01 \mathrm{H}), 6.40(\mathrm{~m}, 0.12 \mathrm{H}), 6.20(\mathrm{~m}, 0.01$

H), 5.87 (br s, $0.13 \mathrm{H}$ ), 5.43 (br s, $0.04 \mathrm{H}$ ), 3.33 (br m, $1.00 \mathrm{H}$ ), 3.07 (br q, $J=6.9 \mathrm{~Hz}, 11.86$

H), 2.92 (br s, $0.14 \mathrm{H}), 2.49$ (s, $2.99 \mathrm{H}), 2.23$ (br m, $0.12 \mathrm{H}), 2.06$ (s, $0.47 \mathrm{H}), 1.74(\mathrm{~s}, 0.22 \mathrm{H})$,

$1.67(\mathrm{~s}, 0.03 \mathrm{H}), 1.16(\mathrm{t}, J=6.9 \mathrm{~Hz}, 20.70 \mathrm{H})$

${ }^{31}$ P NMR: $\quad\left(202 \mathrm{MHz}, d_{6}\right.$-DMSO)

$33.28,33.09,32.72,32.51,32.30$ (major peak), 31.76, 31.54, 31.26, 31.06, 28.29, 25.36, $25.05,22.32$ 
IR: $(\mathrm{KBr} ;$ pressed under Ar)

3169 (m), 3055 (m), 2986 (m), 2943 (m), 2680 (m), 2492 (m), 1673 (m), 1586 (m), 1531 (s),

1507 (s), 1478 (s), 1437 (s), 1399 (m), 1384 (m), 1311 (m), 1056 (s, v br), 872 (w), 847 (w),

$815(\mathrm{~m}), 746(\mathrm{~m}), 697$ (s), $668(\mathrm{~m}), 619(\mathrm{w}), 609(\mathrm{w}), 582(\mathrm{w}), 552(\mathrm{w}), 523(\mathrm{~m}), 501(\mathrm{~m}), 475$

(w)

\section{New Preparation of Dinuclear Palladium Catalysts 4}

To a two-necked, 15-mL, pear-shaped flask charged with a suspension of sodium hydride (5.4 $\mathrm{mg}, 226 \mu \mathrm{mol}, 2$ equiv) in anhydrous acetonitrile $(1.0 \mathrm{~mL})$ at $0{ }^{\circ} \mathrm{C}$ was added 1 -phenylhexane-1,3,5trione $(23 \mathrm{mg}, 113 \mu \mathrm{mol})$ portion wise under Ar, and the mixture stirred at $\mathrm{rt}$ for $15 \mathrm{~min}$. The solution turned clear, bright yellow, and $\mathrm{H}_{2}$ evolution was observed. Tetrakis(acetonitrile)palladium(II) tetrafluoroborate (100 mg, $226 \mu \mathrm{mol}, 2$ equiv) was dissolved in anhydrous acetonitrile $(1.5 \mathrm{~mL})$ under Ar in a separate $10-\mathrm{mL}$ Schlenk flask, and cooled to $-20{ }^{\circ} \mathrm{C}$. The solution of deprotonated triketone was added slowly to the palladium-containing solution via cannula at $-20{ }^{\circ} \mathrm{C}$, washing with anhydrous acetonitrile $(0.5 \mathrm{~mL})$. The mixture was brought to $\mathrm{rt}$, and stirred for $30 \mathrm{~min}$. This was accompanied by a color change to orange. The mixture was cooled to $-20{ }^{\circ} \mathrm{C}$, then a solution of bisphosphine ligand (113 umol, 1 equiv) in anhydrous benzene $(0.9 \mathrm{~mL}, 0.2 \mathrm{M}$ in ligand) was added dropwise over $3 \mathrm{~min}$. The reaction mixture was stirred at $\mathrm{rt}$ for $12 \mathrm{~h}$. It was then concentrated under high vacuum $(120 \mathrm{~Pa})$ to a brown powder. This was washed in a glove box with anhydrous $\mathrm{Et}_{2} \mathrm{O}(10 \mathrm{~mL})$, then anhydrous toluene $(3$ $\times 10 \mathrm{~mL}$ ), removing a yellow filtrate. The solids were dissolved in anhydrous acetone to yield a yellowbrown solution, which was quickly filtered to remove a white solid. The filtrate was concentrated under high vacuum $(120 \mathrm{~Pa})$, and washed with anhydrous $\mathrm{Et}_{2} \mathrm{O}(4 \times 10 \mathrm{~mL})$, then anhydrous toluene $(3 \times 10$ $\mathrm{mL}$ ). The solid was collected. The dinuclear palladium catalysts decompose quickly, and decomposition appears to be accelerated under vacuum. The complexes must be kept under inert atmosphere, and should be used and characterized without delay. 


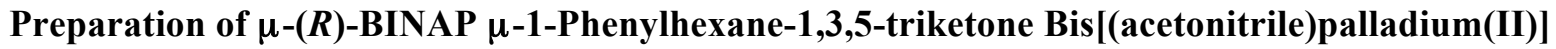

\section{Tetrafluoroborate $4 a$}

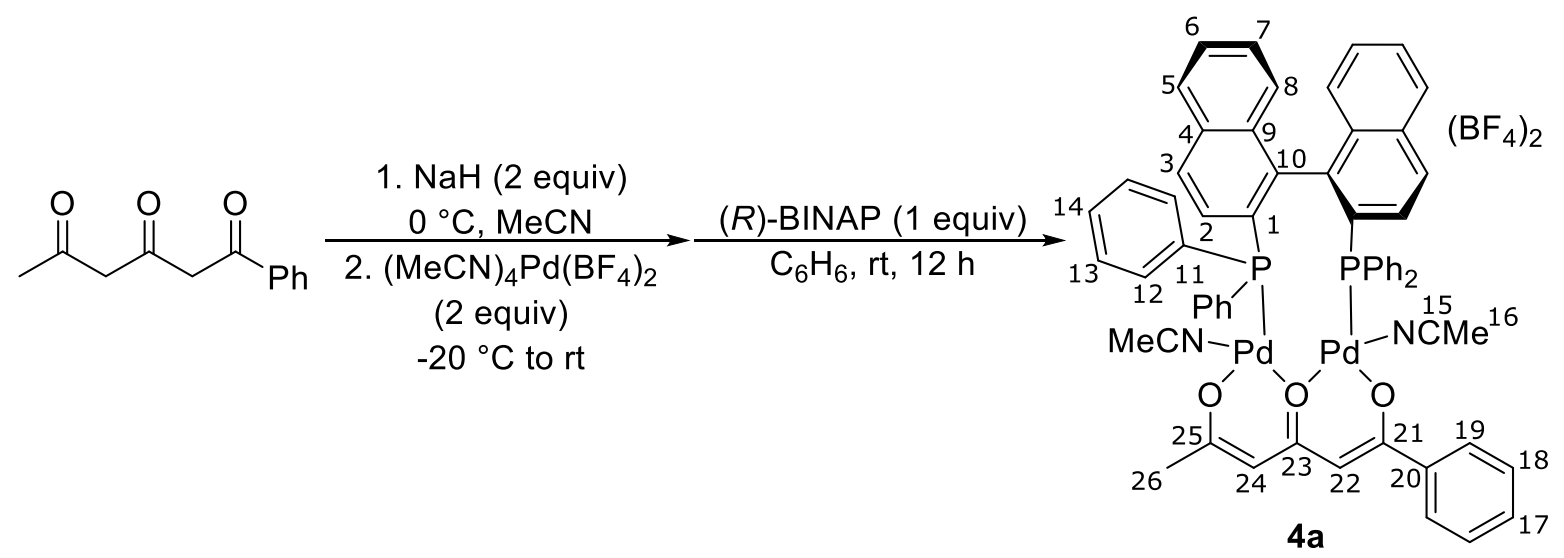

Dinuclear complex 4a was synthesized from tetrakis(acetonitrile)palladium(II) tetrafluoroborate (226 umol), producing $131 \mathrm{mg}(101 \mu \mathrm{mol}, \mathbf{9 0 \%}$ yield) of a yellow-brown powder.

Data for 4a:

${ }^{1} \mathrm{H}$ NMR: $\quad\left(500 \mathrm{MHz}, d_{6}\right.$-DMSO)

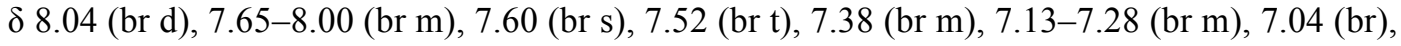

6.72-6.95 (br m), 6.54 (br d, $J=6.2 \mathrm{~Hz}$ ), 6.35 (br), 2.28 (s), 2.06 (s), 1.82 (s), 1.74 (s), 1.67

(s), $1.07\left(\mathrm{t}, \mathrm{H}_{3} \mathrm{C}(16)\right)$

${ }^{31}$ P NMR: $\quad\left(202 \mathrm{MHz}, d_{6}\right.$-DMSO)

33.10

IR: (powder, under air)

3694 (w), 3434 (br w), 2924.16 (w), 1439 (w), 1376 (w), 1084 (w), 1023 (w), 1003 (w), $833(w), 697(w), 558(w), 539(w), 498(w), 470(w)$ 
Preparation of $\mu$-(R)-Tol-BINAP $\mu$-1-Phenylhexane-1,3,5-triketone Bis[(acetonitrile)palladium(II)] Tetrafluoroborate $4 \mathrm{~b}$

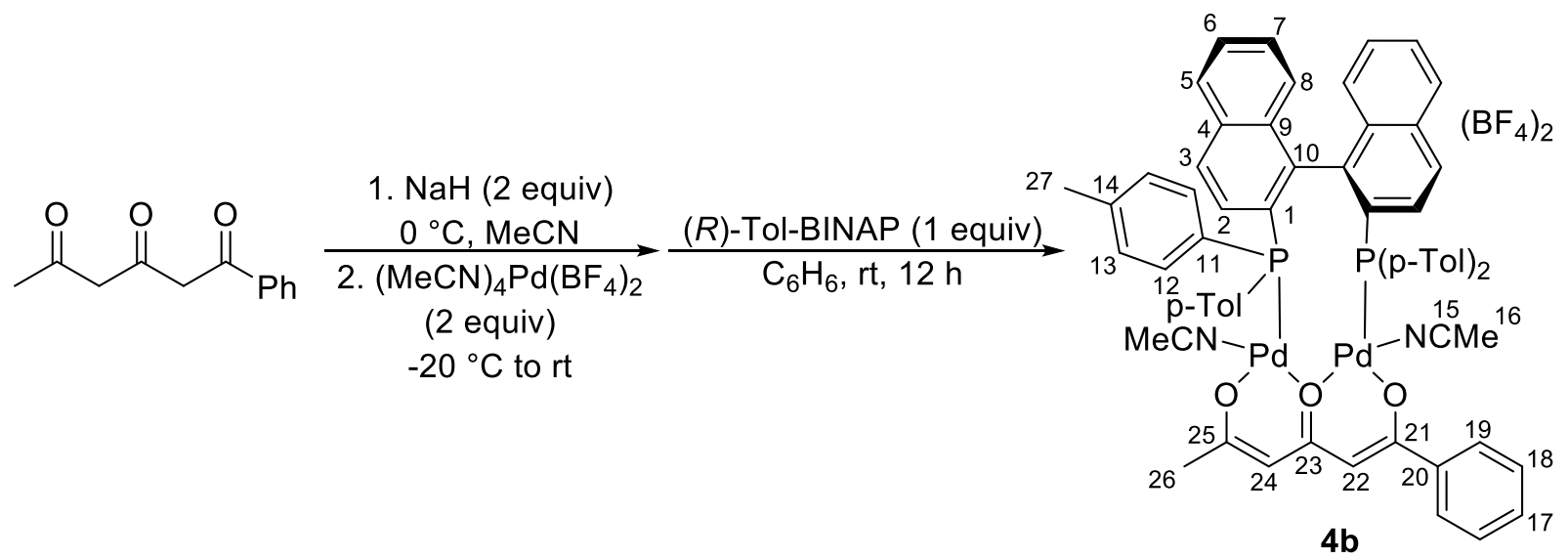

Dinuclear complex $\mathbf{4 b}$ was synthesized from tetrakis(acetonitrile)palladium(II) tetrafluoroborate (108 $\mu \mathrm{mol})$ to yield $64 \mathrm{mg}(47 \mu \mathrm{mol}, \mathbf{8 8 \%}$ yield $)$ of a light brown powder.

Data for $\mathbf{4 b}$ :

${ }^{1} \mathrm{H}$ NMR: $\quad\left(500 \mathrm{MHz}, d_{6}\right.$-DMSO)

$\delta 8.04(\mathrm{~d}, J=7.5 \mathrm{~Hz}), 7.89(\mathrm{~d}, J=8.6 \mathrm{~Hz}), 7.73-7.87(\mathrm{~m}), 7.35-7.70(\mathrm{~m}), 7.12-7.32(\mathrm{~m})$, 7.09 (t, $J=7.9 \mathrm{~Hz}), 6.79$ (d, $J=8.5 \mathrm{~Hz}), 6.49-6.75$ (m), 6.40 (s), 6.31 (s), 5.73 (s), 2.07 (s), $2.06(\mathrm{~s}), 1.74\left(\mathrm{~s}, \mathrm{H}_{3, \mathrm{~A}} \mathrm{C}(27)\right), 1.64\left(\mathrm{~s}, \mathrm{H}_{3, \mathrm{~B}} \mathrm{C}(27)\right)$

${ }^{31}$ P NMR: $\quad\left(202 \mathrm{MHz}, d_{6}\right.$-DMSO) 32.39

IR: (powder, under air) 3427 (br w), 3058 (w), 1590 (w), 1557 (w), 1437 (w), 1313 (w), $1238(w), 1055(w), 1022(w)$, $997(w), 745(w), 696(w), 523(w), 498(w)$ 


\section{Preparation of Racemic Dibromide Standards}

\section{Preparation of $r a c-6 a, r a c-6 b$ and $r a c-6 d$}

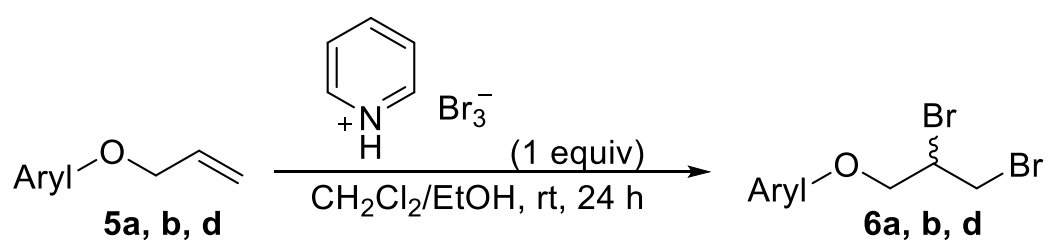

A $20-\mathrm{mL}$ scintillation vial was charged with allylic ether $5(0.50 \mathrm{mmol})$ and $\mathrm{CH}_{2} \mathrm{Cl}_{2}(1.5 \mathrm{~mL}$, $0.33 \mathrm{M}$ in 5). Pyridinium tribromide ( $88 \%$ active, $182 \mathrm{mg}, 0.50 \mathrm{mmol}, 1.0$ equiv) was then added in one portion, followed by $95 \%$ ethanol $(1.5 \mathrm{~mL}, 0.33 \mathrm{M}$ in $\mathbf{5})$. The vial was capped with a plastic-lined cap, and the orange-yellow solution stirred for $24 \mathrm{~h}$, during which time the color is lost. Reaction progress was monitored by TLC. The reaction was quenched by the addition of saturated aqueous $\mathrm{Na}_{2} \mathrm{~S}_{2} \mathrm{O}_{3}$ solution $(1.0 \mathrm{~mL})$, and the mixture diluted with $\mathrm{CH}_{2} \mathrm{Cl}_{2}(10 \mathrm{~mL})$ and $\mathrm{H}_{2} \mathrm{O}(10 \mathrm{~mL})$. The layers were separated, and the organic phase was extracted with $\mathrm{CH}_{2} \mathrm{Cl}_{2}(3 \times 10 \mathrm{~mL})$. The organic extracts were dried over $\mathrm{MgSO}_{4}$, filtered, and concentrated in vacuo to give racemic dibromides 6.

\section{Preparation of rac-1-(2,3-Dibromopropoxy)-4-Methoxybenzene (6a)}

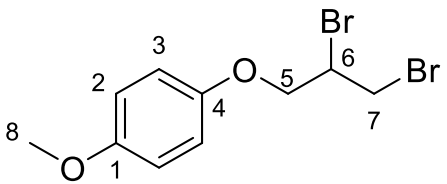

Dibromide 6a was synthesized from $\mathbf{5 a}(0.3 \mathrm{mmol})$ to yield $94 \mathrm{mg}(290 \mu \mathrm{mol}, \mathbf{9 7 \%}$ yield $)$ of a white solid. Spectroscopic data were consistent with those of Henry. ${ }^{1}$

Data for 6a:

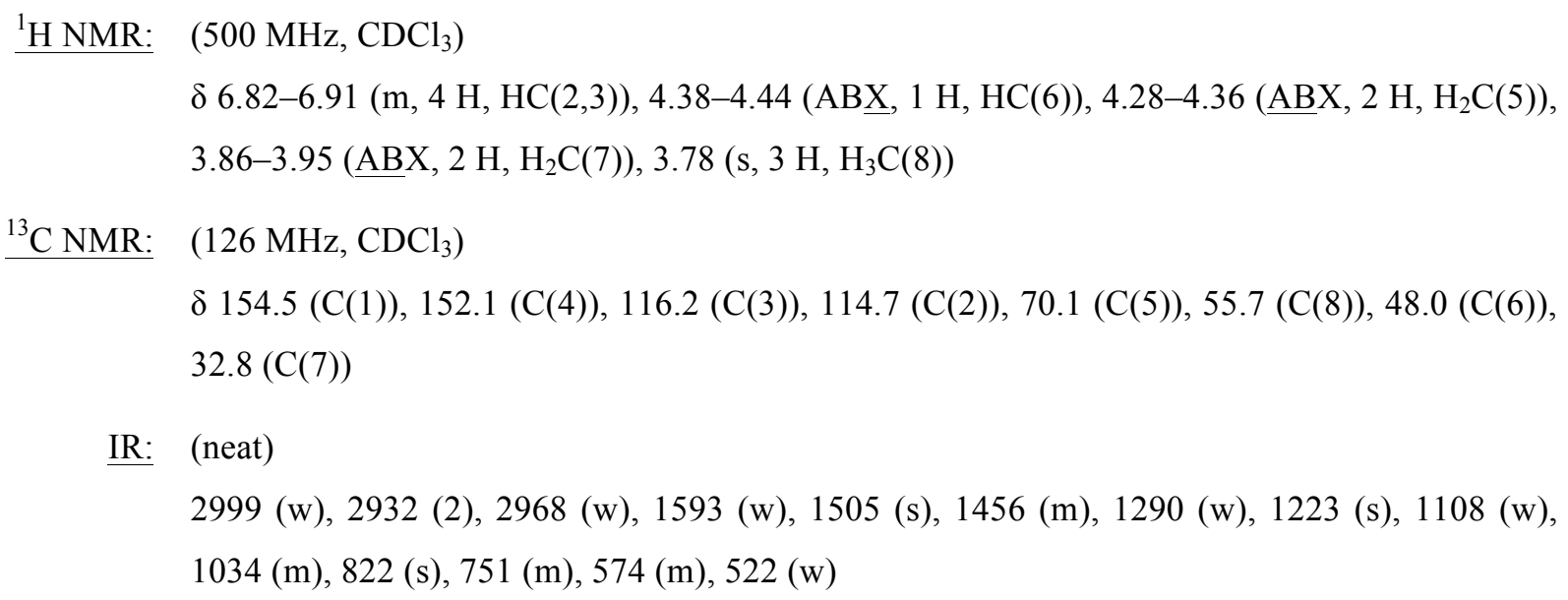


MS: (EI)

$325.9\left(11, \mathrm{M}^{+}\right), 323.9\left(20, \mathrm{M}^{+}\right), 321.9\left(11, \mathrm{M}^{+}\right), 164.1\left(9,\left[\mathrm{M}-\mathrm{Br}_{2}\right]^{+}\right), 124.1$ (90), 123.1 (100, $\left.\left[\mathrm{M}-\mathrm{C}_{3} \mathrm{H}_{5} \mathrm{Br}_{2}\right]^{+}\right), 109.0(15), 95.0(19), 62.0(11)$

TLC: $\quad R_{f} 0.36(90: 10$ hexane/EtOAc) $[\mathrm{UV} / \mathrm{CAM}]$

HPLC: $\quad t_{\mathrm{R}} 19.6 \min (49.8 \%) ; t_{\mathrm{R}} 21.2 \min (50.2 \%)\left(\mathrm{OJ}-\mathrm{H}\right.$, hexane $/ i-\mathrm{PrOH}=95.5: 4.5,1.0 \mathrm{~mL} \min ^{-1}, 220$ $\left.\mathrm{nm}, 20^{\circ} \mathrm{C}\right)$

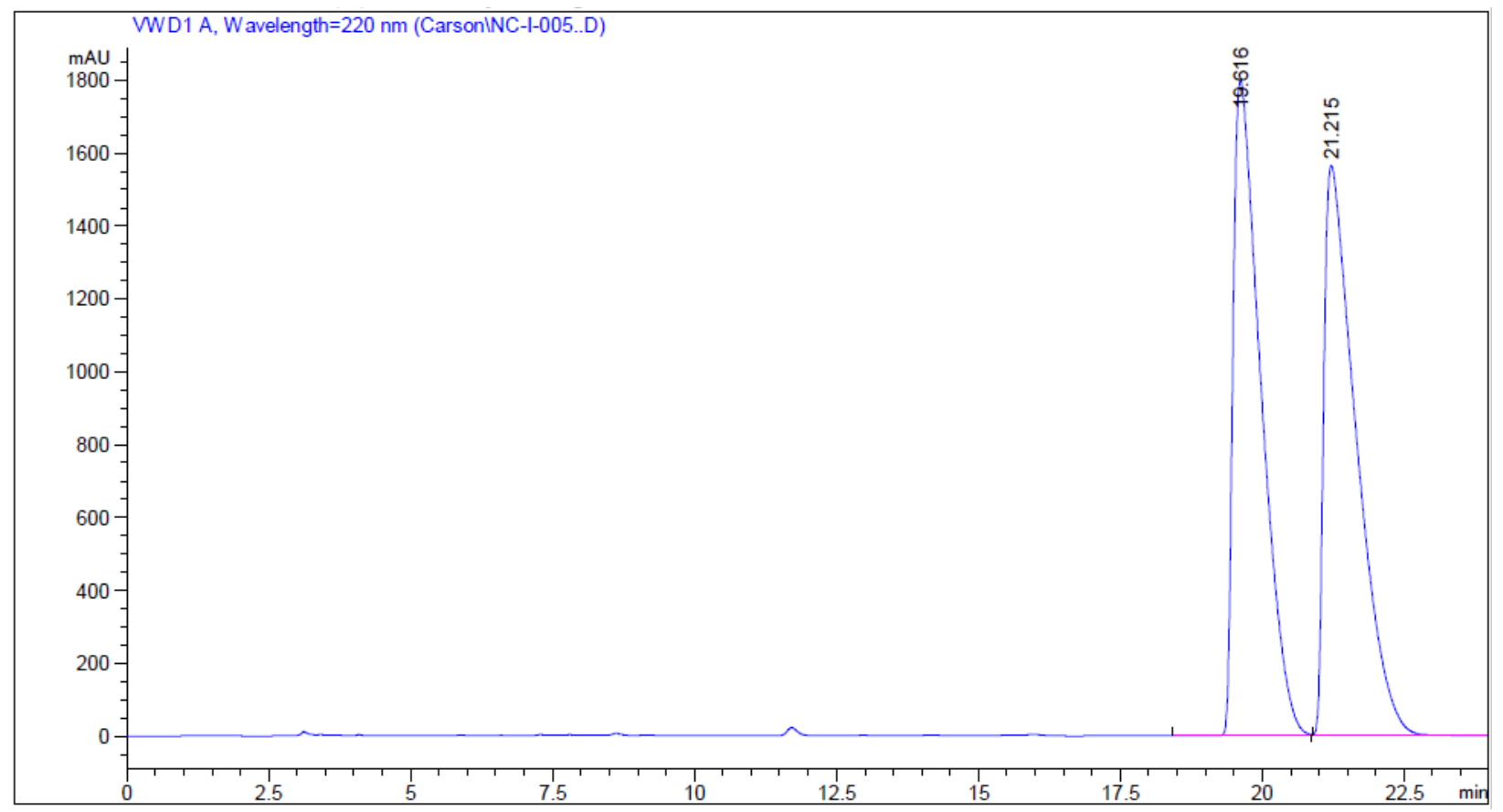

Preparation of 1-(2,6-Dibromopropoxy)-4-Cyanobenzene (6b)<smiles>N#Cc1ccc(OCC(Br)CBr)cc1</smiles>

Dibromide $\mathbf{6 b}$ was synthesized from $\mathbf{5 b}(0.3 \mathrm{mmol})$ to yield $92 \mathrm{mg}(288 \mu \mathrm{mol}, \mathbf{9 6 \%}$ yield $)$ of a white solid. Spectroscopic data were consistent with those of Henry. ${ }^{1}$

Data for $6 \mathbf{b}$ :

${ }^{1} \mathrm{H}$ NMR: $\quad\left(500 \mathrm{MHz}, \mathrm{CDCl}_{3}\right)$

$\delta 7.61(\mathrm{~d}, J=9.0 \mathrm{~Hz}, 2 \mathrm{H}, \mathrm{HC}(2)), 7.00(\mathrm{~d}, J=9.0 \mathrm{~Hz}, 2 \mathrm{H}, \mathrm{HC}(3)), 4.39-4.48(\mathrm{~m}, 3 \mathrm{H}$, $\left.\mathrm{H}_{2} \mathrm{C}(5), \mathrm{HC}(6)\right), 3.87-3.93$ (m, 2 H, $\mathrm{H}_{2} \mathrm{C}(7)$ ) 
${ }^{13} \mathrm{C}$ NMR: $\quad\left(126 \mathrm{MHz}, \mathrm{CDCl}_{3}\right)$

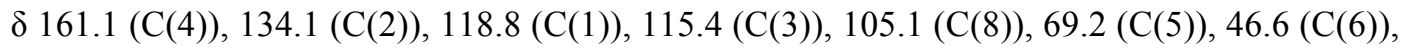
$32.1(\mathrm{C}(7))$

IR: (powder)

2924 (w), 2227 (m), 1904 (w), 1651 (w), 1605 (m), 1509 (m), 1453 (w), 1302 (w), 1270 (m), 1253 (m), 1173 (m), 1119 (w), 1043 (w), 990 (w), 834 (m), 575 (m), 546 (m)

MS: (EI)

$320.9\left(11, \mathrm{M}^{+}\right), 318.9\left(22, \mathrm{M}^{+}\right), 316.9\left(11, \mathrm{M}^{+}\right), 240.0\left(2,[\mathrm{M}-\mathrm{HBr}]^{+}\right), 238.0\left(2,[\mathrm{M}-\mathrm{HBr}]^{+}\right)$, 202.9 (39, [M-OAryl ${ }^{+}$], 200.9 (82, [M-OAryl $\left.{ }^{+}\right], 198.9$ (42, [M-OAryl ${ }^{+}$], 120.9 (36, $\left.[\mathrm{M}-\mathrm{BrOAryl}]^{+}\right), 119.0\left(100,[\mathrm{M}-\mathrm{BrOAryl}]^{+}\right), 102.0\left(14,\left[\mathrm{C}_{6} \mathrm{H}_{4} \mathrm{CN}\right]^{+}\right), 90.0(8), 62.1$ (45)

TLC: $\quad R_{f} 0.15\left(90: 10\right.$ hexane/EtOAc) $\left[\mathrm{AgNO}_{3}-\mathrm{H}_{2} \mathrm{O}_{2}\right]$

HPLC: $t_{\mathrm{R}} 30.5 \min (50.0 \%) ; t_{\mathrm{R}} 32.8 \mathrm{~min}(50.0 \%)\left(\mathrm{IB}-3\right.$, hexane $/ i-\mathrm{PrOH}=92: 8,1.0 \mathrm{~mL} \mathrm{~min}{ }^{-1}$, $\left.220 \mathrm{~nm}, 20^{\circ} \mathrm{C}\right)$

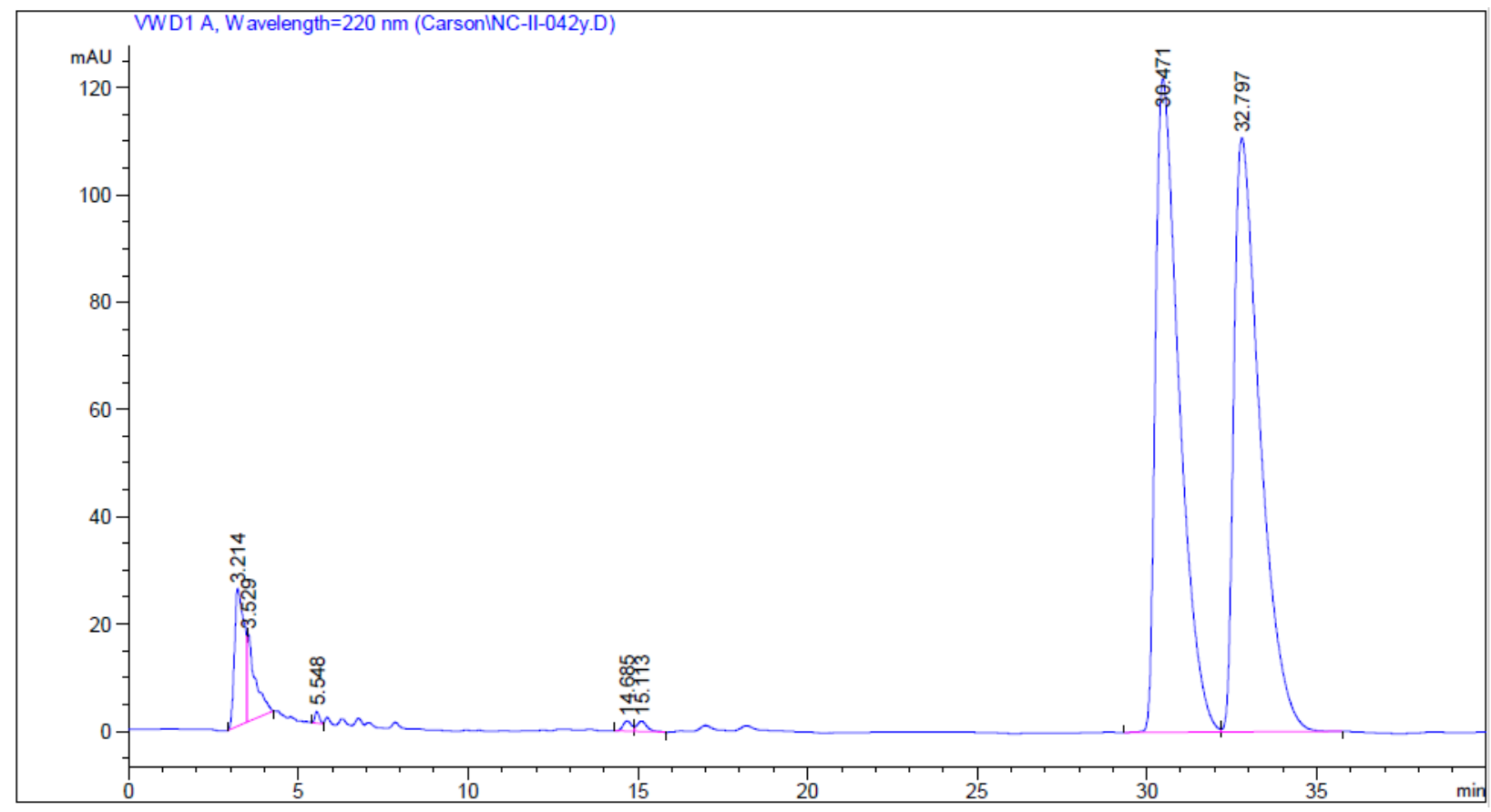




\section{Preparation of rac-1-(2,3-Dibromopropoxy)-2,6-Diisopropylbenzene (6d)}

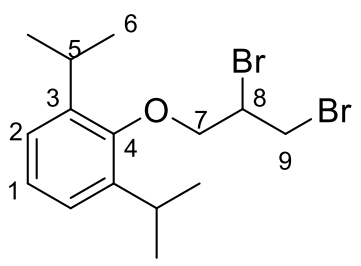

Dibromide 6d was synthesized from $\mathbf{5 d}(0.2 \mathrm{mmol})$ to yield $73 \mathrm{mg}(193 \mu \mathrm{mol}, \mathbf{9 7 \%}$ yield $)$ of colorless needles. Spectroscopic data were consistent with those of Henry. ${ }^{1}$

Data for 6d:

1 H NMR: $\quad\left(500 \mathrm{MHz}, \mathrm{CDCl}_{3}\right)$

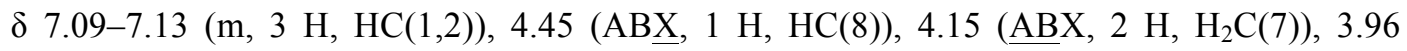
( $\left.\underline{\mathrm{ABX}}, 2 \mathrm{H}, \mathrm{H}_{2} \mathrm{C}(9)\right), 3.42$ (sept, $\left.J=6.8 \mathrm{~Hz}, 2 \mathrm{H}, \mathrm{HC}(5)\right), 1.24$ (d, $\left.J=6.8 \mathrm{~Hz}, 12 \mathrm{H}, \mathrm{H}_{3} \mathrm{C}(6)\right)$

${ }^{13} \mathrm{C}$ NMR: $\quad\left(126 \mathrm{MHz}, \mathrm{CDCl}_{3}\right)$

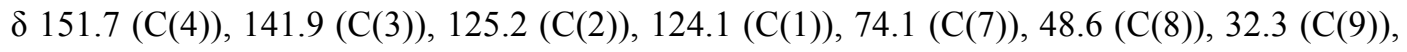
$26.3(\mathrm{C}(5)), 24.1(\mathrm{C}(6))$

IR: (neat) $2962(\mathrm{w}), 2927(\mathrm{w}), 2868(\mathrm{w}), 1589(\mathrm{w}), 1457$ (w), $1442(\mathrm{w}), 1328(\mathrm{w}), 1256(\mathrm{w}), 1180(\mathrm{~m})$, 1035 (w), $981(\mathrm{w}), 914(\mathrm{w}), 799(\mathrm{w}), 759(\mathrm{~m}), 577$ (m)

MS: (EI)

$380.0\left(11, \mathrm{M}^{+}\right), 378.0\left(22, \mathrm{M}^{+}\right), 376.0\left(11, \mathrm{M}^{+}\right), 218.2(5), 202.9\left(6,[\mathrm{M}-\text { Aryl }]^{+}\right), 200.9$ (12, [M-Aryl $\left.]^{+}\right), 198.9\left(6,[\mathrm{M}-\text { Aryl }]^{+}\right), 178.1\left(100,\left[\mathrm{M}-\mathrm{C}_{3} \mathrm{H}_{4} \mathrm{Br}_{2}\right]^{+}\right), 163.1$ (92), 147.1 (10), 135.1 (47), 121.0 (12), 107.1 (13), 91.1 (17), 77.1 (7), 62.1 (6)

TLC: $\quad R_{f} 0.60(90: 10$ hexane/EtOAc) [UV/CAM]

HPLC: $\quad t_{\mathrm{R}} 12.5 \min (49.7 \%) ; t_{\mathrm{R}} 13.9 \min (50.3 \%)\left(\mathrm{IB}-3\right.$, hexane $/ i-\mathrm{PrOH}=99.9: 0.1,1.0 \mathrm{~mL} \mathrm{~min}^{-1}, 220$ $\left.\mathrm{nm}, 20^{\circ} \mathrm{C}\right)$ 


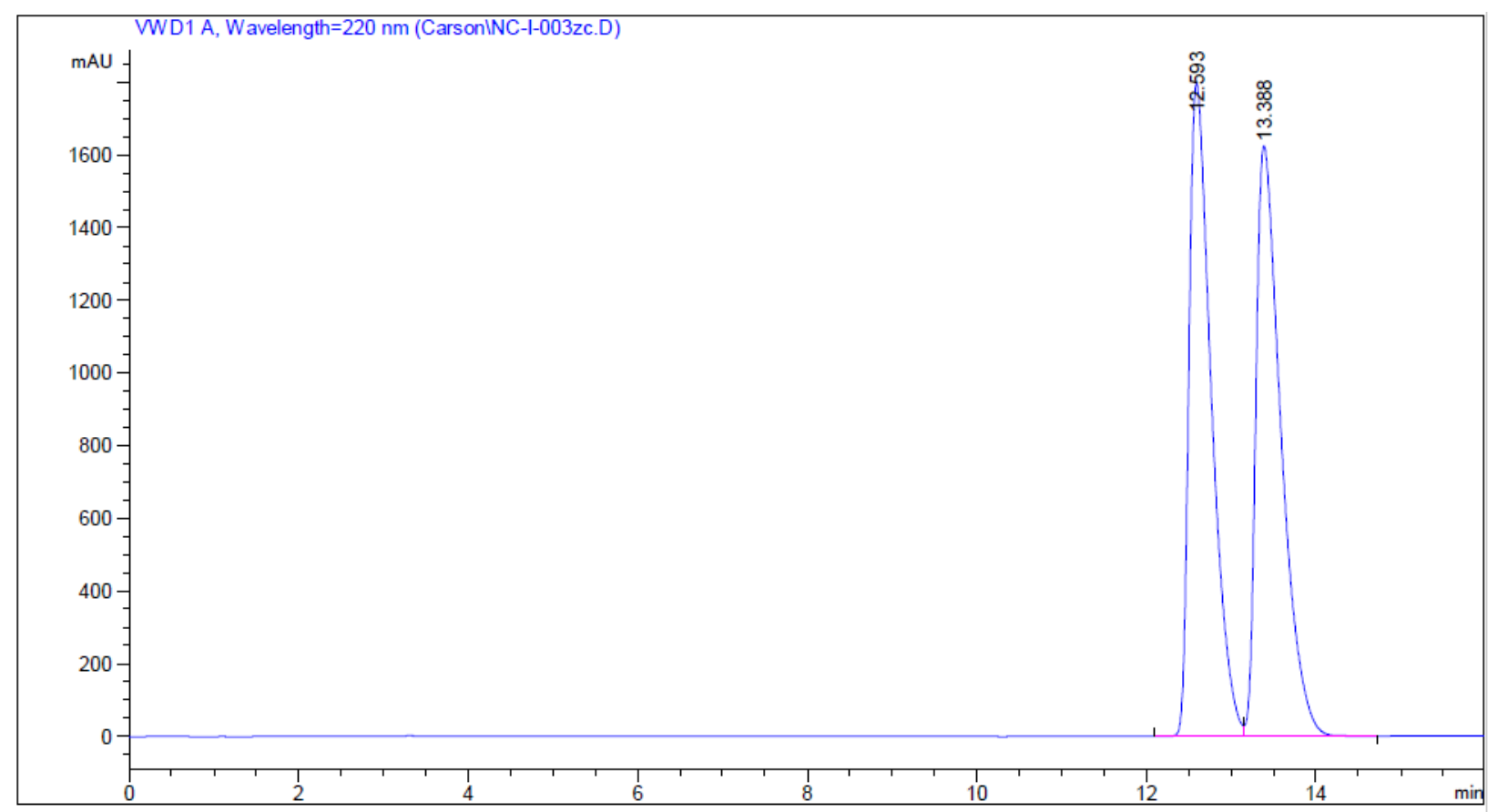

Preparation of rac-(2,3-Dibromopropoxy)benzene (6c)

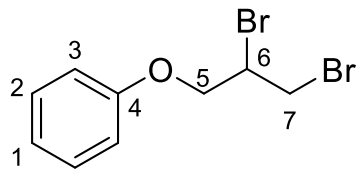

Dibromide 6c was prepared according to a literature procedure, ${ }^{7}$ and had spectroscopic data consistent with the literature.

Data for $\mathbf{6 c}$ :

${ }^{1} \mathrm{H}$ NMR: $\quad\left(500 \mathrm{MHz}, \mathrm{CDCl}_{3}\right)$

$\delta 7.31(\mathrm{dd}, J=8.5,7.5 \mathrm{~Hz}, 2 \mathrm{H}, \mathrm{HC}(2)), 7.00$ (t, $J=7.4 \mathrm{~Hz}, 1 \mathrm{H}, \mathrm{HC}(1)), 6.94$ (d, $J=7.9 \mathrm{~Hz}$,

$2 \mathrm{H}, \mathrm{HC}(3)), 4.35-4.47$ (m, $\left.3 \mathrm{H}, \mathrm{H}_{2} \mathrm{C}(6), \mathrm{HC}(7)\right), 3.88-3.96\left(\mathrm{~m}, 2 \mathrm{H}, \mathrm{H}_{2} \mathrm{C}(8)\right)$

${ }^{13} \mathrm{C}$ NMR: $\quad\left(126 \mathrm{MHz}, \mathrm{CDCl}_{3}\right)$

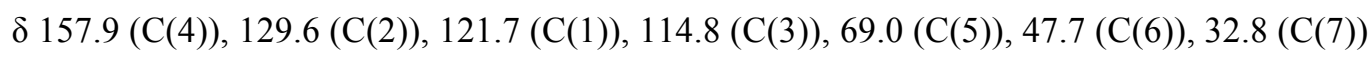

IR: (neat)

2927 (w), 2869 (w), 1598 (m), 1495 (m), 1240 (m), 1047 (w), 908 (w), 884 (w), 814 (w), $752(\mathrm{~s}), 690(\mathrm{~m}), 509(\mathrm{w})$ 
MS: (EI)

$295.9\left(10, \mathrm{M}^{+}\right), 293.9\left(21, \mathrm{M}^{+}\right), 291.9\left(11, \mathrm{M}^{+}\right), 202.9\left(6,[\mathrm{M}-\mathrm{OPh}]^{+}\right), 200.9\left(13,[\mathrm{M}-\mathrm{OPh}]^{+}\right)$, $198.9\left(7,[\mathrm{M}-\mathrm{OPh}]^{+}\right), 121.0\left(14,[\mathrm{M}-\mathrm{BrOPh}]^{+}\right), 119.0\left(14,[\mathrm{M}-\mathrm{BrOPh}]^{+}\right), 95.0(11), 94.0$ (100), $77.1\left(16.9, \mathrm{Ph}^{+}\right), 65.1(13)$

TLC: $\quad R_{f} 0.78\left(90: 10\right.$ hexane/Et $\left.{ }_{2} \mathrm{O}\right)[\mathrm{UV} / \mathrm{CAM}]$

SFC: $\quad t_{\mathrm{R}} 6.3 \min (49.8 \%) ; t_{\mathrm{R}} 6.6 \min (50.2 \%)\left(\mathrm{OD}, \mathrm{MeOH}: \mathrm{CO}_{2}=3: 97,2.5 \mathrm{~mL} \mathrm{~min}{ }^{-1}, 220 \mathrm{~nm}, 40\right.$ $\left.{ }^{\circ} \mathrm{C}\right)$

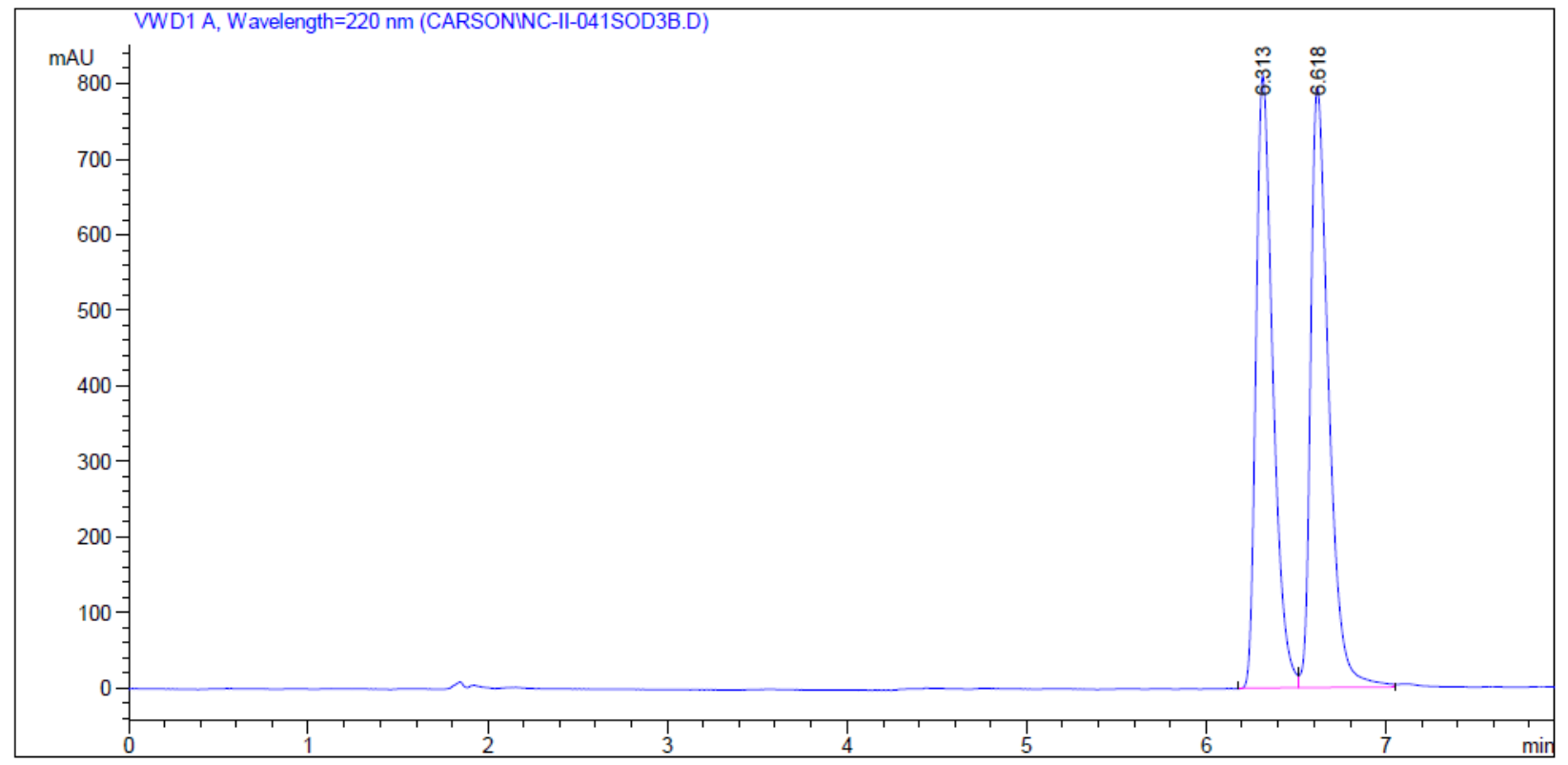

\section{Preparation of Racemic Chlorohydrin Standards}

\section{Preparation of $r a c-2 a$ and $r a c-2 c$}

Racemic standards of chlorohydrins $\mathbf{2 a}$ and $\mathbf{2 c}$ were prepared according to a literature procedure ${ }^{8}$ and had spectroscopic data consistent with literature. 


\section{Preparation of rac-(2-Chloro-3-hydroxypropoxy)benzene (2a)}<smiles>OC(CCl)COc1ccccc1</smiles>

Data for 2a:

${ }^{1} \mathrm{H}$ NMR: $\quad\left(500 \mathrm{MHz}, \mathrm{CDCl}_{3}\right)$

$\delta 7.31(\mathrm{~m}, 2 \mathrm{H}, \mathrm{HC}(2)), 7.00$ (tt, $J=7.4,1.0 \mathrm{~Hz}, 1 \mathrm{H}, \mathrm{HC}(1)), 6.93$ (m, $2 \mathrm{H}, \mathrm{HC}(3)), 4.23$ (sext, $J=5.5 \mathrm{~Hz}, 1 \mathrm{H}, \mathrm{HC}(6), 4.10\left(\mathrm{~m}, 2 \mathrm{H}, \mathrm{H}_{2} \mathrm{C}(5)\right), 3.77$ (m, $\left.2 \mathrm{H}, \mathrm{H}_{2} \mathrm{C}(7)\right), 2.59$ (d, $J=5.8 \mathrm{~Hz}, 1 \mathrm{H}$, $\mathrm{OH})$

${ }^{13} \mathrm{C} \mathrm{NMR:} \quad\left(126 \mathrm{MHz}, \mathrm{CDCl}_{3}\right)$

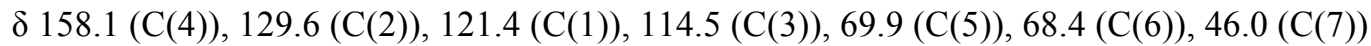

IR: (neat)

3407 (br, w), 2928 (w), 1739 (w), 1599 (m), 1588 (w), 1495 (m), 1457 (w), 1240 (m), 1173 (w), $1078(\mathrm{~m}), 1042$ (m), 751 (s), 690 (m), 509 (m)

MS: (EI)

$188.0\left(22, \mathrm{M}^{+}\right), 186.0\left(59, \mathrm{M}^{+}\right), 144.0(9), 142.0(30), 137.0$ (18), 119.0 (18), $108.0(11), 107.0$

(63), 95.0 (61), 94 (100), 79.1 (20), 78.1 (13), $77.0\left(69, \mathrm{Ph}^{+}\right), 66.1$ (14), 65.1 (18), 51.0 (23)

TLC: $\quad R_{f} 0.78\left(90: 10\right.$ hexane/Et $\left.{ }_{2} \mathrm{O}\right)[\mathrm{UV} / \mathrm{CAM}]$

SFC: $\quad t_{\mathrm{R}} 9.5 \mathrm{~min}(49.8 \%) ; t_{\mathrm{R}} 11.5 \mathrm{~min}(50.2 \%)\left(\mathrm{OD}, \mathrm{MeOH}: \mathrm{CO}_{2}=1: 99\right.$ for $10 \min$ then 10:90 for 6

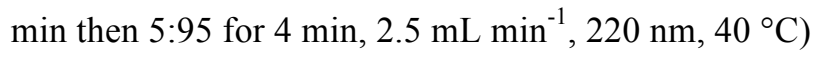

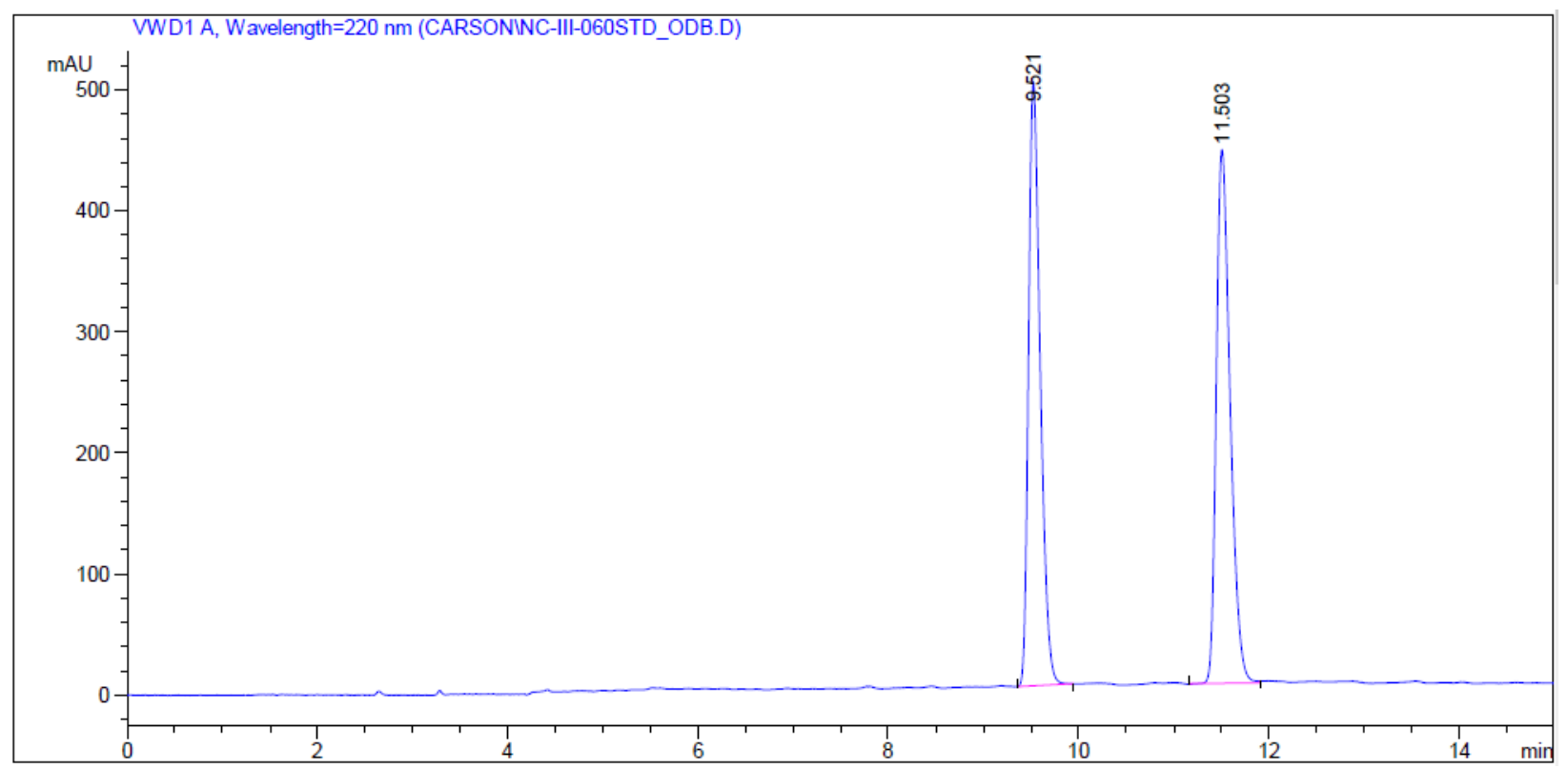




\section{Preparation of rac-1-(2-Chloro-3-hydroxypropoxy)naphthalene (2c)}

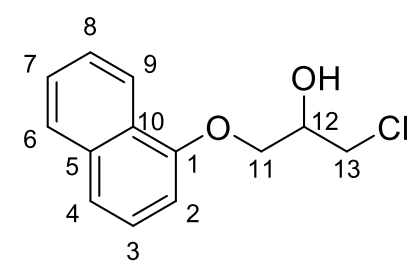

Data for $2 \mathrm{c}$ :

${ }^{1} \mathrm{H}$ NMR: $\quad\left(500 \mathrm{MHz}, \mathrm{CDCl}_{3}\right)$

$\delta 8.21(\mathrm{~d}, J=7.1 \mathrm{~Hz}, 1 \mathrm{H}, \mathrm{HC}(9)), 7.82(\mathrm{~d}, J=6.9 \mathrm{~Hz}, 1 \mathrm{H}, \mathrm{HC}(6)), 7.45-7.54(\mathrm{~m}, 3 \mathrm{H})$, $\mathrm{HC}(4), \mathrm{HC}(7), \mathrm{HC}(8)), 7.39$ (t, $J=7.9 \mathrm{~Hz}, 1 \mathrm{H}, \mathrm{HC}(3)), 6.85$ (d, $J=7.5 \mathrm{~Hz}, 1 \mathrm{H}, \mathrm{HC}(2)), 4.39$ (sext, $J=5.5 \mathrm{~Hz}, 1 \mathrm{H}, \mathrm{HC}(12)), 4.28$ (m, $2 \mathrm{H}, \mathrm{H}_{2} \mathrm{C}(11)$ ), 3.89 (m, $\left.2 \mathrm{H}, \mathrm{H}_{2} \mathrm{C}(13)\right), 2.59$ (d, $J=$ $6.1 \mathrm{~Hz}, \mathrm{OH})$

${ }^{13} \mathrm{C}$ NMR: $\quad\left(126 \mathrm{MHz}, \mathrm{CDCl}_{3}\right)$

ठ $153.7(\mathrm{C}(10)), 134.5(\mathrm{C}(5)), 127.6(\mathrm{C}(6)), 126.5(\mathrm{C}(7)), 125.7(\mathrm{C}(3)), 125.4(\mathrm{C}(8)), 125.3$ $(\mathrm{C}(1)), 121.5(\mathrm{C}(9)), 121.0(\mathrm{C}(4)), 105.0(\mathrm{C}(2)), 69.9(\mathrm{C}(11)), 68.6(\mathrm{C}(12)), 46.3(\mathrm{C}(13))$

IR: (powder) 3312 (br, w), 3056 (w), 2926 (w), 2853 (w), 1738 (w), 1627 (w), 1577 (m), 1509 (w), 1456 (w), 1393 (m), 1322 (w), 1270 (m), 1208 (m), 1100 (m), 1021 (m), 904 (m), 793 (m), 770 (s), $707(\mathrm{~m}), 574(\mathrm{~m}), 514(\mathrm{w})$

MS: (EI)

$238.0\left(8, \mathrm{M}^{+}\right), 236.0\left(25, \mathrm{M}^{+}\right), 145.0(13), 144.0(100), 127.0\left(10, \mathrm{Naph}^{+}\right), 116(15), 115$ (25), $94.0(10)$

TLC: $\quad R_{f} 0.78\left(90: 10\right.$ hexane/ $\left./ \mathrm{Et}_{2} \mathrm{O}\right)[\mathrm{UV} / \mathrm{CAM}]$

SFC: $t_{\mathrm{R}} 24.2 \min (50.2 \%) ; t_{\mathrm{R}} 32.1 \mathrm{~min}(49.8 \%)\left(\mathrm{OD}, \mathrm{MeOH}: \mathrm{CO}_{2}=10: 90,2.5 \mathrm{~mL} \mathrm{~min}^{-1}, 220 \mathrm{~nm}, 40\right.$ $\left.{ }^{\circ} \mathrm{C}\right)$ 


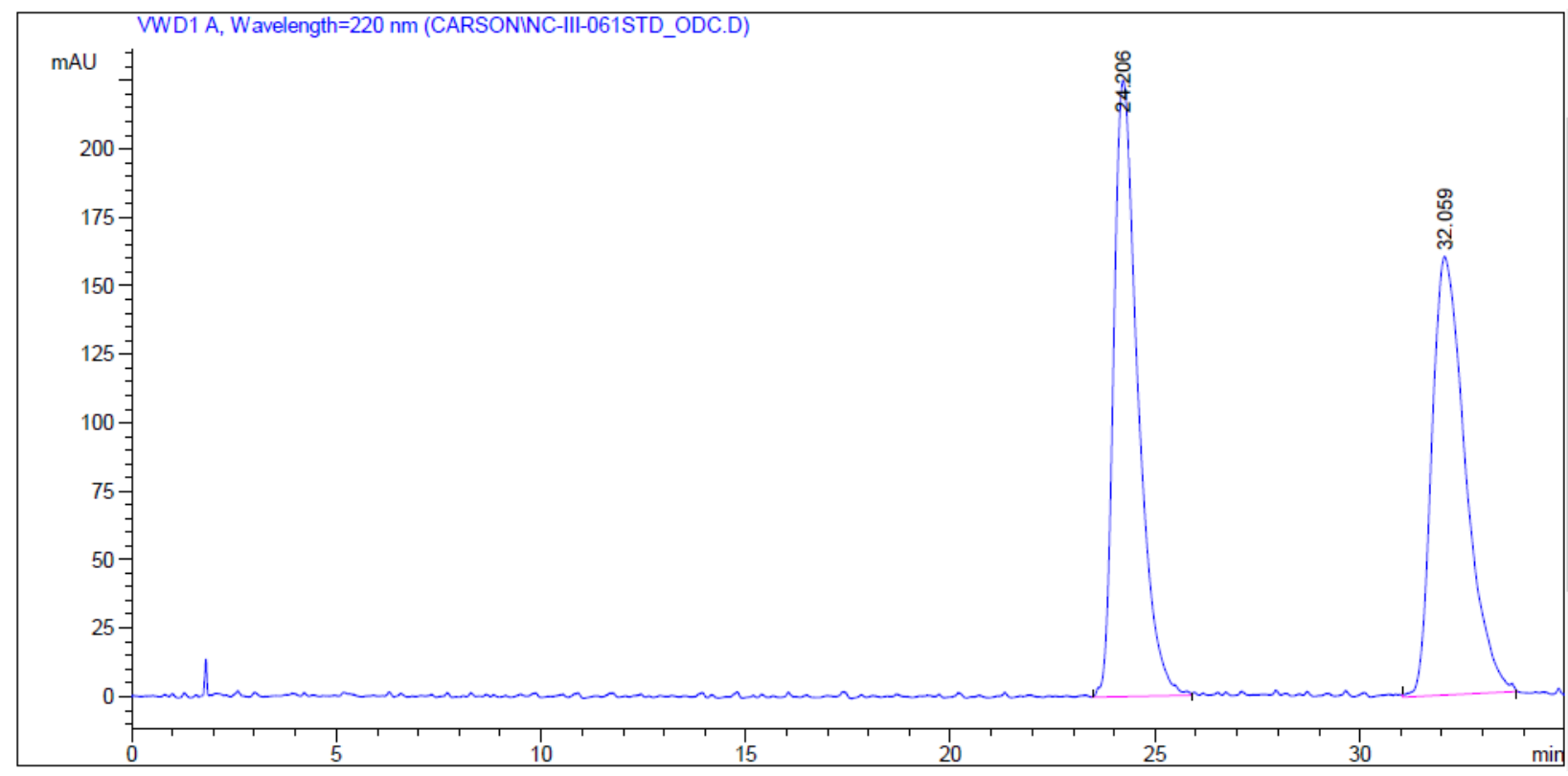

Preparation of rac-4-Chloro-3-hydroxybutan-2-one (2b)

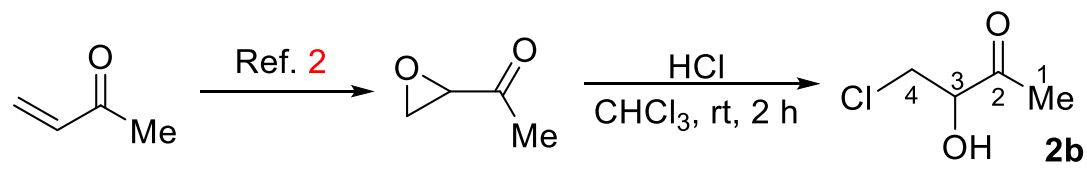

1-(Oxiran-2-yl)ethanone was prepared on a $2 \mathrm{mmol}$ scale, according to a literature procedure. ${ }^{9}$ The crude, colorless oil produced in this reaction was then cooled to $0{ }^{\circ} \mathrm{C}$ in a $7-\mathrm{mL}$ vial, and $\mathrm{CHCl}_{3}(0.5$ $\mathrm{mL})$ and concentrated $\mathrm{HCl}(0.25 \mathrm{~mL})$ were added to form a colorless/yellow biphase. The mixture was stirred at $\mathrm{rt}$ for $2 \mathrm{~h} . \mathrm{H}_{2} \mathrm{O}(3 \mathrm{~mL})$ was added, and the organic components were extracted with $\mathrm{CHCl}_{3}(3 \times 5$ $\mathrm{mL}$ ). The organic extracts were dried over $\mathrm{CaCl}_{2}$, filtered, and concentrated in vacuo to a crude, dark, brown oil. This complex mixture was separated via column chromatography using an eluent gradient from 95:5 to 80:20 hexane/ethyl acetate, to yield $71 \mathrm{mg}(0.58 \mathrm{mmol}, \mathbf{2 9} \%$ yield over two steps $)$ of a volatile, colorless oil $\mathbf{2 b}$.

Data for $\mathbf{2 b}$ :

${ }^{1} \mathrm{H}$ NMR: $\quad\left(500 \mathrm{MHz}, \mathrm{CDCl}_{3}\right)$

$\delta 4.29(\mathrm{t}, J=5.6 \mathrm{~Hz}, 1 \mathrm{H}, \mathrm{HC}(3)), 3.92\left(\mathrm{~m}, 2 \mathrm{H}, \mathrm{H}_{2} \mathrm{C}(4)\right), 3.02($ br s, $1 \mathrm{H}, \mathrm{OH}), 2.33$ (s, 3

$\left.\mathrm{H}, \mathrm{H}_{3} \mathrm{C}(1)\right)$ 


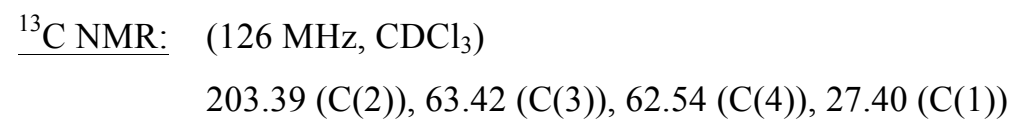

IR: (neat)

3419 (br, w), 2941 (w), 1715 (s), 1420 (m), 1358 (m), 1224 (m), 1162 (w), 1075 (m), 1041

(m), $929(\mathrm{w}), 796(\mathrm{w}), 733(\mathrm{~m}), 680(\mathrm{~m}), 629(\mathrm{~m}), 593(\mathrm{~m}), 559(\mathrm{~m}), 474(\mathrm{~s})$

MS: (EI)

128.0 (10), 126.0 (16), 109.1 (7.4, [M-Me] $\left.]^{+}\right), 107.0$ (23.0, [M-Me] $\left.{ }^{+}\right), 94.0$ (12), 92.0

(37.6), 91.1 (11.9), $87.1\left(20.2,[\mathrm{M}-\mathrm{Cl}]^{+}\right), 86.1$ (13.5), 81.1 (16.3, [M-Ac] $\left.]^{+}\right), 79.0$ (53.8, [M-

$\mathrm{Ac}^{+}$), 77.0 (17.2), 76.0 (11.2), 71.1 (21.2), 69.1 (15.2), 64.1 (47.7), 62.1 (100), 57.2 (15.9),

$55.1(14.2)$

TLC: $\quad R_{f} 0.37(70: 30$ hexane/EtOAc) [DNP]

GC: $\quad t_{\mathrm{R}} 16.6 \min (50.3 \%) ; t_{\mathrm{R}} 17.4 \min (49.7 \%)\left(\right.$ CycloSil-B, $\left.70^{\circ} \mathrm{C}\right)$

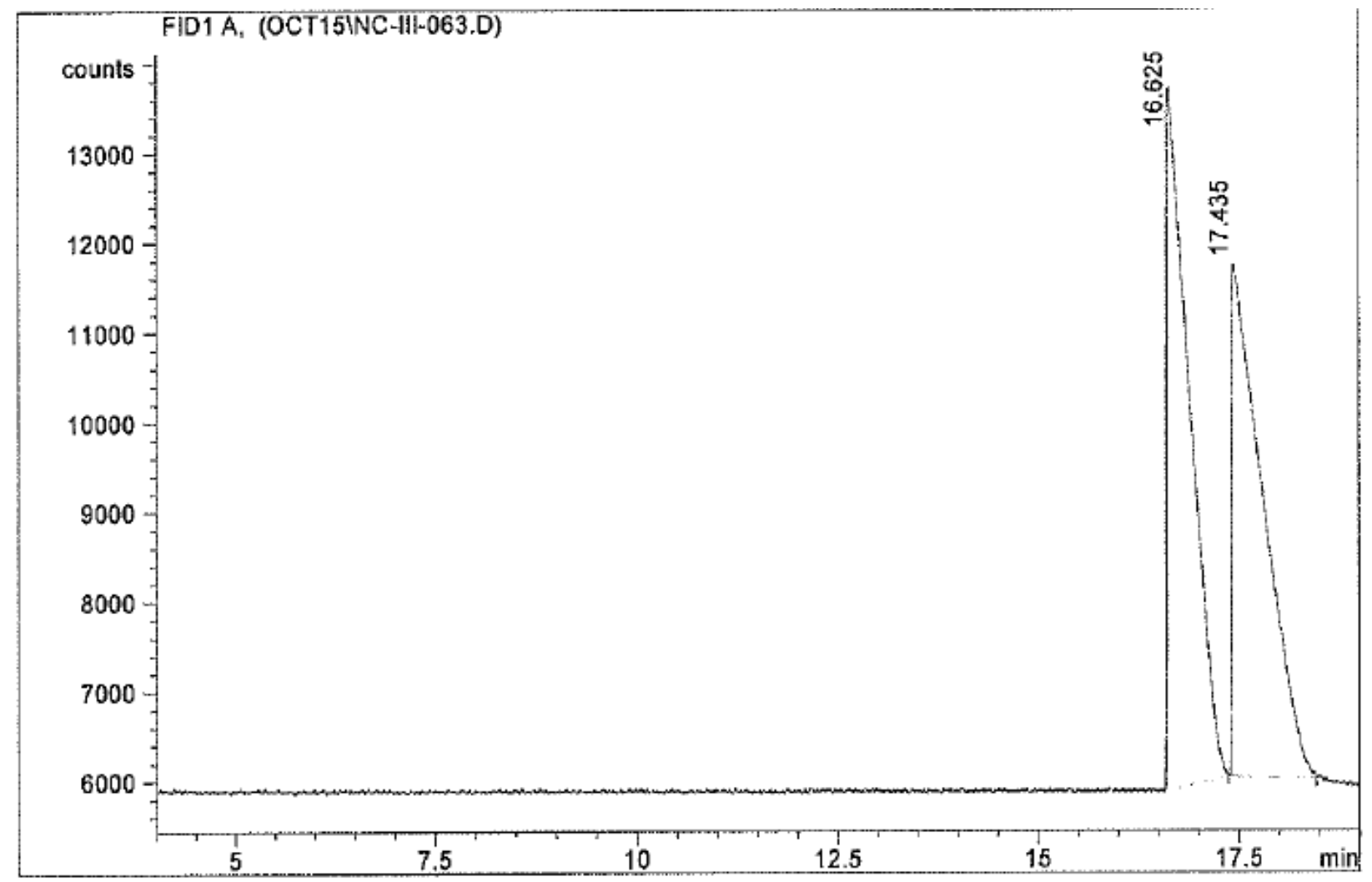

\section{Conditions and Spectra for Dibromination Reactions:}

Reactions were run according to Henry's procedures. ${ }^{1}$ The procedures were all reproduced as rigorously as the described procedures allow, other than changing the scale and reaction time - with the 
exception of Run 3. Reaction progress was monitored by TLC. Concentrations are calculated with respect to total solvent volume.

Table S2. Conditions for Dibromination Reactions

\begin{tabular}{|c|c|c|c|c|c|c|c|c|c|}
\hline Run & Substrate & $\begin{array}{c}\text { Amount of } \\
\text { substrate, } \\
\text { mmol }\end{array}$ & $\begin{array}{c}\mathbf{P d} \\
\mathbf{c o m p l e x} \\
\mathbf{m o l} \mathbf{\%}\end{array}$ & $\begin{array}{c}\mathbf{C u B r}_{\mathbf{2}} \\
\mathbf{e q u i v}\end{array}$ & $\begin{array}{c}\mathbf{C u B r}_{2} \\
\mathbf{m o l a r i t y}, \\
\mathbf{M}\end{array}$ & $\begin{array}{c}\mathbf{L i B r} \\
\mathbf{e q u i v}\end{array}$ & $\begin{array}{c}\mathbf{L i B r} \\
\mathbf{m o l a r i t y}, \\
\mathbf{M}\end{array}$ & $\begin{array}{c}\mathrm{THF} / \mathrm{H}_{2} \mathrm{O} \\
\mathbf{T}\end{array}$ & $\begin{array}{c}\text { Time, } \\
\mathbf{h}\end{array}$ \\
\hline $\mathbf{1}$ & $\mathbf{5 a}^{\dagger}$ & 0.25 & 2.3 & 13.74 & $2.0 \mathrm{M}$ & 1.75 & $0.26 \mathrm{M}$ & $17: 3$ & 28 \\
\hline $\mathbf{2}$ & $\mathbf{5 a}^{\dagger}$ & 0.25 & 2.3 & 13.74 & $2.0 \mathrm{M}$ & 1.75 & $0.26 \mathrm{M}$ & $17: 3$ & 28 \\
\hline $\mathbf{3}$ & $\mathbf{5 a}^{\dagger}$ & 3.0 & 2.3 & 13.73 & $2.1 \mathrm{M}$ & 1.73 & $0.26 \mathrm{M}$ & $17: 3$ & 144 \\
\hline $\mathbf{4}$ & $\mathbf{5 a}^{\dagger}$ & 0.25 & 2.3 & 13.75 & $2.1 \mathrm{M}$ & 1.75 & $0.27 \mathrm{M}$ & $17: 3$ & 6 \\
\hline $\mathbf{5}$ & $\mathbf{5 b}$ & 0.25 & 2.4 & 15.83 & $2.0 \mathrm{M}$ & 1.01 & $0.13 \mathrm{M}$ & $1: 9$ & 24 \\
\hline $\mathbf{6}$ & $\mathbf{5 c}$ & 0.25 & 1.4 & 10.65 & $2.0 \mathrm{M}$ & 1.11 & $0.20 \mathrm{M}$ & $17: 3$ & 24 \\
\hline $\mathbf{7}$ & $\mathbf{5 d}$ & 0.25 & 2.5 & 15.83 & $2.2 \mathrm{M}$ & 1.24 & $0.17 \mathrm{M}$ & $87: 13$ & 24 \\
\hline
\end{tabular}

${ }^{\dagger}$ Nuclearity of palladium(II) catalyst is ambiguous in the original text for dibromination of $\mathbf{5 a}$. 
Run 1: Dibromination of 5a, 3.00 mmol, 6 days $\mathrm{s}^{\dagger}$

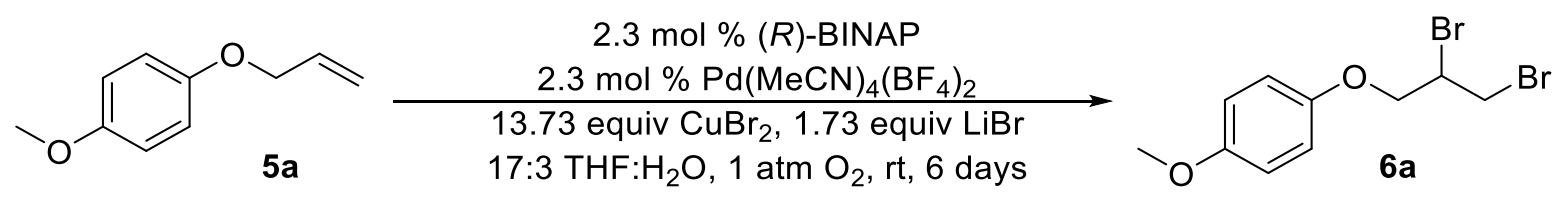

An oven-dried, 50-mL, round-bottomed flask containing a Teflon-coated, magnetic stir bar was charged with tetrakis(acetonitrile)palladium(II) tetrafluoroborate (31 mg, $69 \mu \mathrm{mol}, 2.3 \mathrm{~mol} \%$ ) and (R)2,2'-bis(diphenylphosphino)-1,1'-binaphthyl ((R)-BINAP) (43 mg, $69 \mu \mathrm{mol}, 2.3 \mathrm{~mol} \%$ ) in a glove box. The system was sealed under Ar, and removed from the glove box, then an Ar line inserted, and 17:3 $\mathrm{THF} / \mathrm{H}_{2} \mathrm{O}(15 \mathrm{~mL})$ injected. The solution was stirred at $\mathrm{rt}$ for $10 \mathrm{~min}$, and turned from light to dark orange color. Then, copper(II) bromide (9.20 g, $41.2 \mathrm{mmol}, 13.73$ equiv, $2.1 \mathrm{M})$ was added in one portion to form a deep green solution, followed by lithium bromide ( $450 \mathrm{mg}, 5.2 \mathrm{mmol}, 1.73$ equiv, $0.26 \mathrm{M}$ ) in one portion, which gave slight yellowing of the solution. The atmosphere was replaced with $1 \mathrm{~atm} \mathrm{O}_{2}$ by sparging, and the reaction run under 1 atm $\mathrm{O}_{2}$ from an oxygen manifold. 5a (493 $\mathrm{mg}, 3.00 \mathrm{mmol}$ ) was then added to the mixture, washing with 17:3 THF/ $\mathrm{H}_{2} \mathrm{O}(5 \mathrm{~mL})$. The mixture was left to stir at rt. After 6 days, the mixture was extracted with $\mathrm{CH}_{2} \mathrm{Cl}_{2}(3 \times 50 \mathrm{~mL})$, then dried over $\mathrm{MgSO}_{4}$. Hexane $(15 \mathrm{~mL})$ was added, and the precipitated orange Pd complex removed by filtration, washing with hexane $(3 \times 10 \mathrm{~mL})$. Solvent was removed in vacuo, then the mixture purified via column chromatography using 95:5 hexane/ethyl acetate as eluent, to yield dibromide 6a, $754 \mathrm{mg}$ (2.33 mmol, 78\% yield) of a white solid. This was found to be racemic by CSP-HPLC. ${ }^{1} \mathrm{H}$ NMR spectroscopic data were identical to those of the racemic standard.

Data for 6a:

HPLC: $\quad t_{\mathrm{R}} 18.7 \min (49.9 \%) ; t_{\mathrm{R}} 20.1 \min (50.1 \%)\left(\mathrm{OJ}-\mathrm{H}\right.$, hexane $/ i-\mathrm{PrOH}=95.5: 4.5,1.0 \mathrm{~mL} \min ^{-1}, 220$ $\left.\mathrm{nm}, 20^{\circ} \mathrm{C}\right)$ 

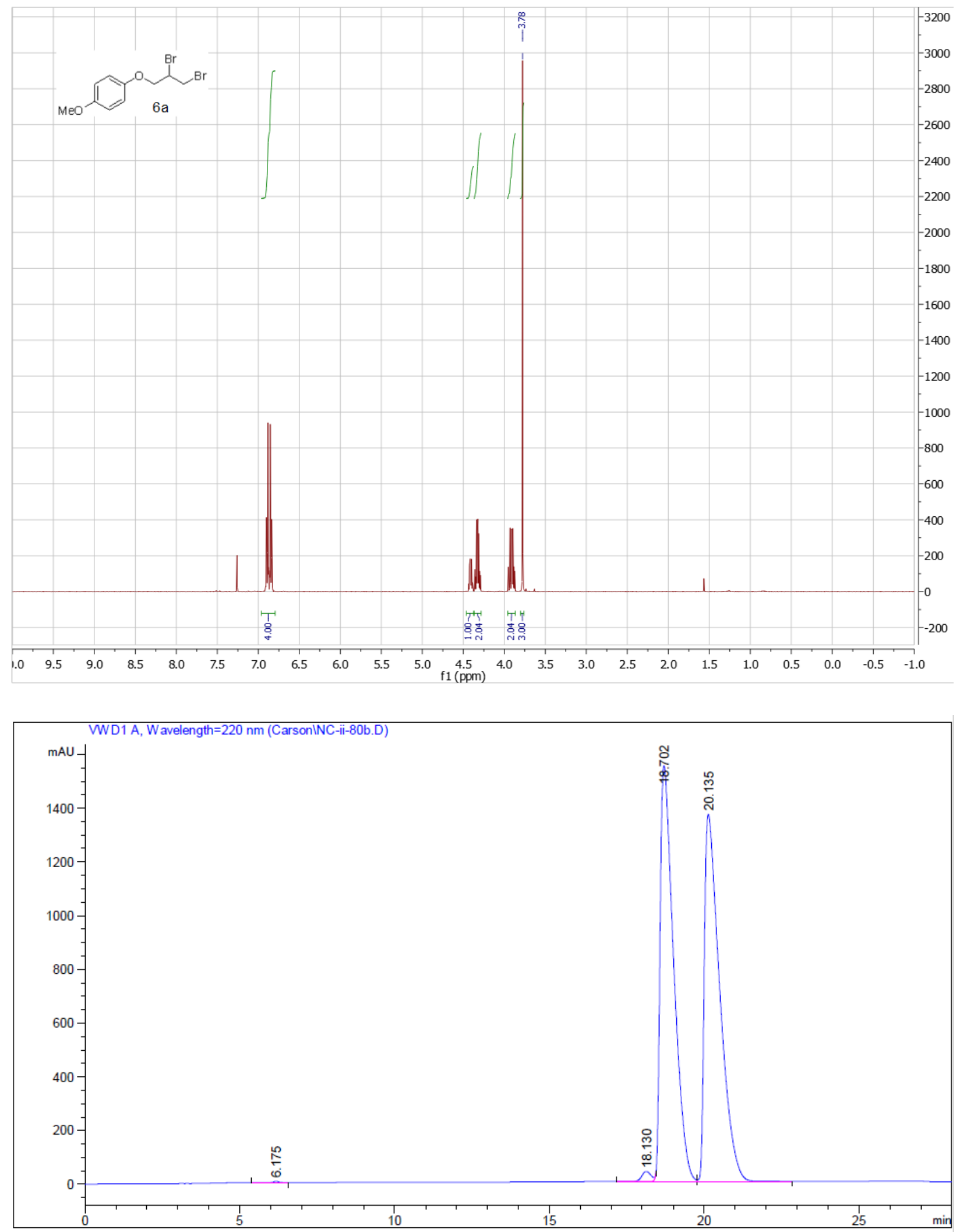
Run 2: Dibromination of 5a, $0.25 \mathrm{mmol}, 6 \mathrm{~h}^{\dagger}$

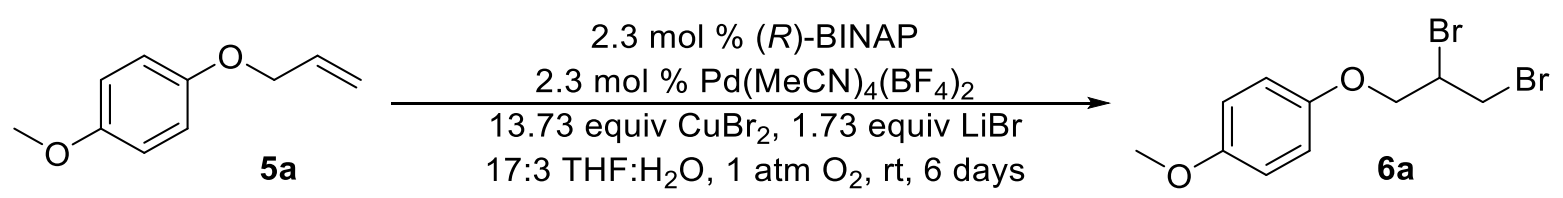

An oven-dried, 15-mL, round-bottomed flask containing a Teflon-coated, magnetic stir bar was charged with tetrakis(acetonitrile)palladium(II) tetrafluoroborate $(2.6 \mathrm{mg}, 5.7 \mu \mathrm{mol}, 2.3 \mathrm{~mol} \%)$ and $(R)$ 2,2'-bis(diphenylphosphino)-1,1'-binaphthyl ((R)-BINAP) (3.6 mg, $5.7 \mu \mathrm{mol}, 2.3 \mathrm{~mol} \%)$ in a glove box. The system was sealed under Ar, and removed from the glove box, then an Ar line inserted, and 17:3 $\mathrm{THF} / \mathrm{H}_{2} \mathrm{O}(1.0 \mathrm{~mL})$ injected. The solution was stirred at $\mathrm{rt}$ for $10 \mathrm{~min}$, and turned from light to dark orange color. Then, copper(II) bromide (758 mg, $3.43 \mathrm{mmol}, 13.75$ equiv, $2.1 \mathrm{M}$ ) was added in one portion to form a deep green solution, followed by lithium bromide ( $38 \mathrm{mg}, 0.43 \mathrm{mmol}, 1.75$ equiv, 0.27 M) in one portion, which gave slight yellowing of the solution. The atmosphere was replaced with 1 atm $\mathrm{O}_{2}$ by sparging, and the reaction run under $1 \mathrm{~atm} \mathrm{O}_{2}$ from an oxygen manifold. 5a (41 $\mathrm{mg}, 0.25 \mathrm{mmol}$ ) was then added to the mixture, washing with $17: 3 \mathrm{THF} / \mathrm{H}_{2} \mathrm{O}(0.6 \mathrm{~mL})$. The mixture was left to stir at $\mathrm{rt}$, and stopped after $6 \mathrm{~h}$. The mixture was extracted with $\mathrm{CH}_{2} \mathrm{Cl}_{2}(3 \times 15 \mathrm{~mL})$, then dried over $\mathrm{MgSO}_{4}$. Hexane $(5 \mathrm{~mL})$ was added, and the precipitated orange Pd complex removed by filtration, washing with hexane $(3 \times 5 \mathrm{~mL})$. Solvent was removed in vacuo, then the mixture purified via column chromatography using 93:7 hexane/ethyl acetate as eluent, to yield dibromide $\mathbf{6 a}, 54 \mathrm{mg}(0.17 \mathrm{mmol}, \mathbf{6 7 \%}$ yield $)$ as a white solid. This was found to be racemic by CSP-HPLC. ${ }^{1} \mathrm{H}$ NMR spectroscopic data were identical to those of the racemic standard.

Data for 6a:

HPLC: $\quad t_{\mathrm{R}} 19.6 \min (50.3 \%) ; t_{\mathrm{R}} 21.1 \min (49.7 \%)\left(\mathrm{OJ}-\mathrm{H}\right.$, hexane $/ i-\mathrm{PrOH}=95.5: 4.5,1.0 \mathrm{~mL} \min ^{-1}, 220$ $\left.\mathrm{nm}, 20^{\circ} \mathrm{C}\right)$ 

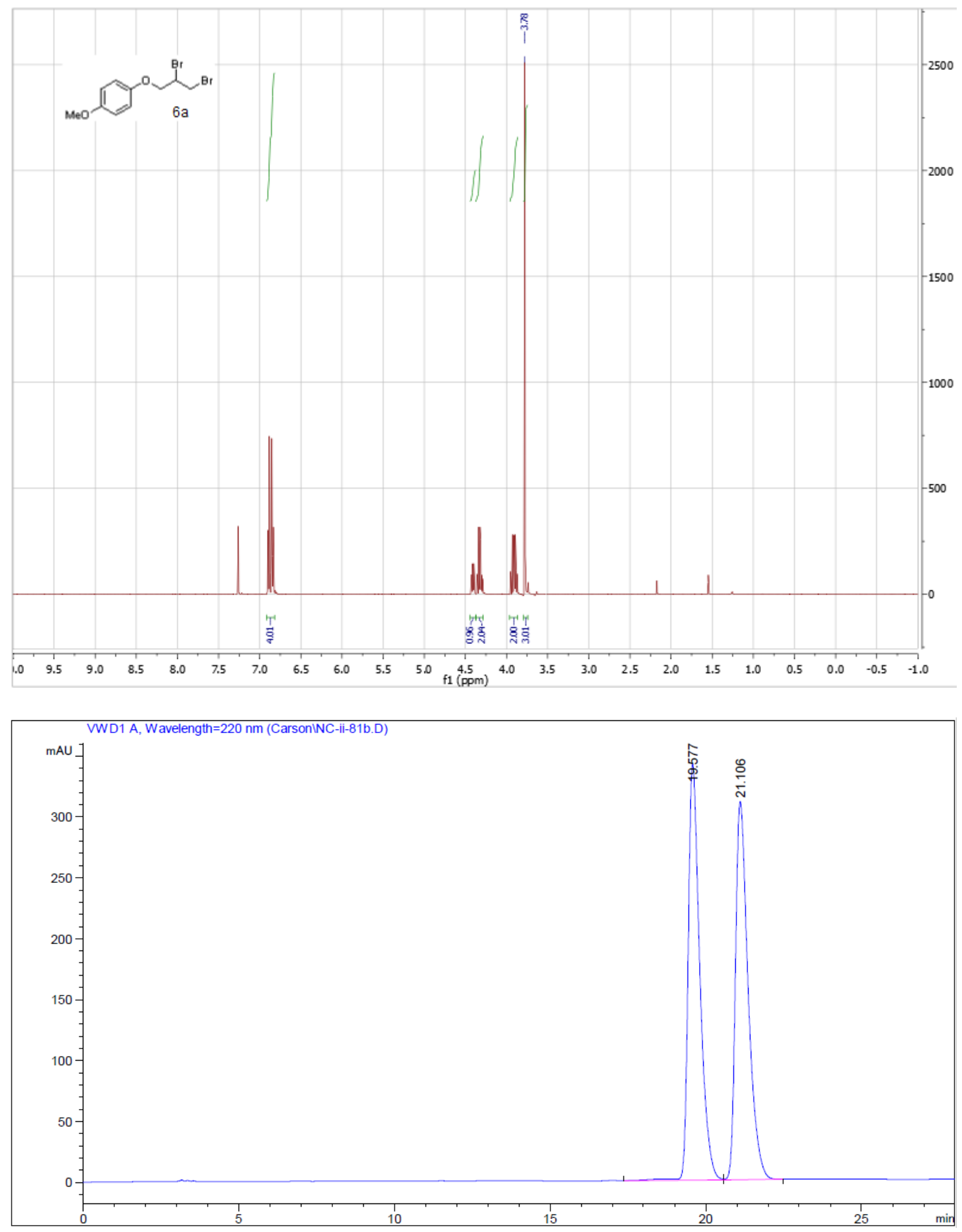
Run 3: Dibromination of 5a with Henry's catalyst preparation, $0.25 \mathrm{mmol}, 28 \mathrm{~h}^{\dagger}$

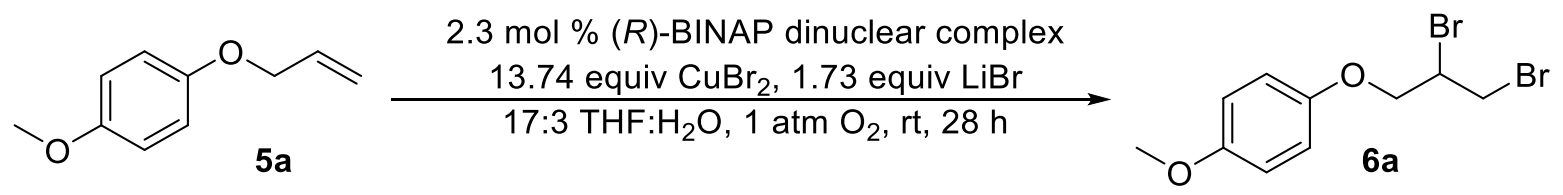

A 25-mL, round-bottomed flask containing a Teflon-coated, magnetic stir bar was charged with 4a ${ }^{\dagger}$ freshly prepared according to Henry's method (7.5 mg, $5.8 \mu \mathrm{mol}, 2.3 \mathrm{~mol} \%$ ), and weighed out in a glove box. The system was sealed under Ar, and removed from the glove box, then an Ar line inserted, and 17:3 THF/ $\mathrm{H}_{2} \mathrm{O}(1.0 \mathrm{~mL})$ injected to give a yellow solution. Then, copper(II) bromide (767 mg, 3.43 mmol, 13.74 equiv, $2.0 \mathrm{M}$ ) was added in one portion to form a deep green solution, followed by lithium bromide (38 mg, $0.43 \mathrm{mmol}, 1.73$ equiv, $0.26 \mathrm{M}$ ) in one portion, which gave slight yellowing of the solution. The atmosphere was replaced with 1 atm $\mathrm{O}_{2}$ by sparging, and the reaction run under $1 \mathrm{~atm} \mathrm{O}_{2}$ from an oxygen manifold. 5a (41 $\mathrm{mg}, 0.25 \mathrm{mmol})$ was then added to the mixture, washing with 17:3 $\mathrm{THF} / \mathrm{H}_{2} \mathrm{O}(0.7 \mathrm{~mL})$. The mixture was left to stir at $\mathrm{rt}$, monitoring periodically by TLC. Completion was reached at $28 \mathrm{~h}$, and the mixture was extracted with $\mathrm{CH}_{2} \mathrm{Cl}_{2}(3 \times 20 \mathrm{~mL})$, then dried over $\mathrm{MgSO}_{4}$. Hexane $(5 \mathrm{~mL})$ was added, and the precipitated orange Pd complex removed by filtration, washing with hexane ( 3 $\times 5 \mathrm{~mL}$ ). Solvent was removed in vacuo, then the mixture purified via column chromatography using 93:7 hexane/ethyl acetate as eluent, to yield dibromide $\mathbf{6 a}$ as a white solid, $70 \mathrm{mg}(0.22 \mathrm{mmol}, \mathbf{8 6 \%}$ yield). This was found to be racemic by CSP-HPLC. ${ }^{1} \mathrm{H}$ NMR spectroscopic data were identical to those of the racemic standard.

Data for 6a:

HPLC: $\quad t_{\mathrm{R}} 19.5 \min (50.1 \%) ; t_{\mathrm{R}} 21.0 \min (49.9 \%)\left(\mathrm{OJ}-\mathrm{H}\right.$, hexane $/ i-\mathrm{PrOH}=95.5: 4.5,1.0 \mathrm{~mL} \min ^{-1}, 220$ $\left.\mathrm{nm}, 20^{\circ} \mathrm{C}\right)$ 

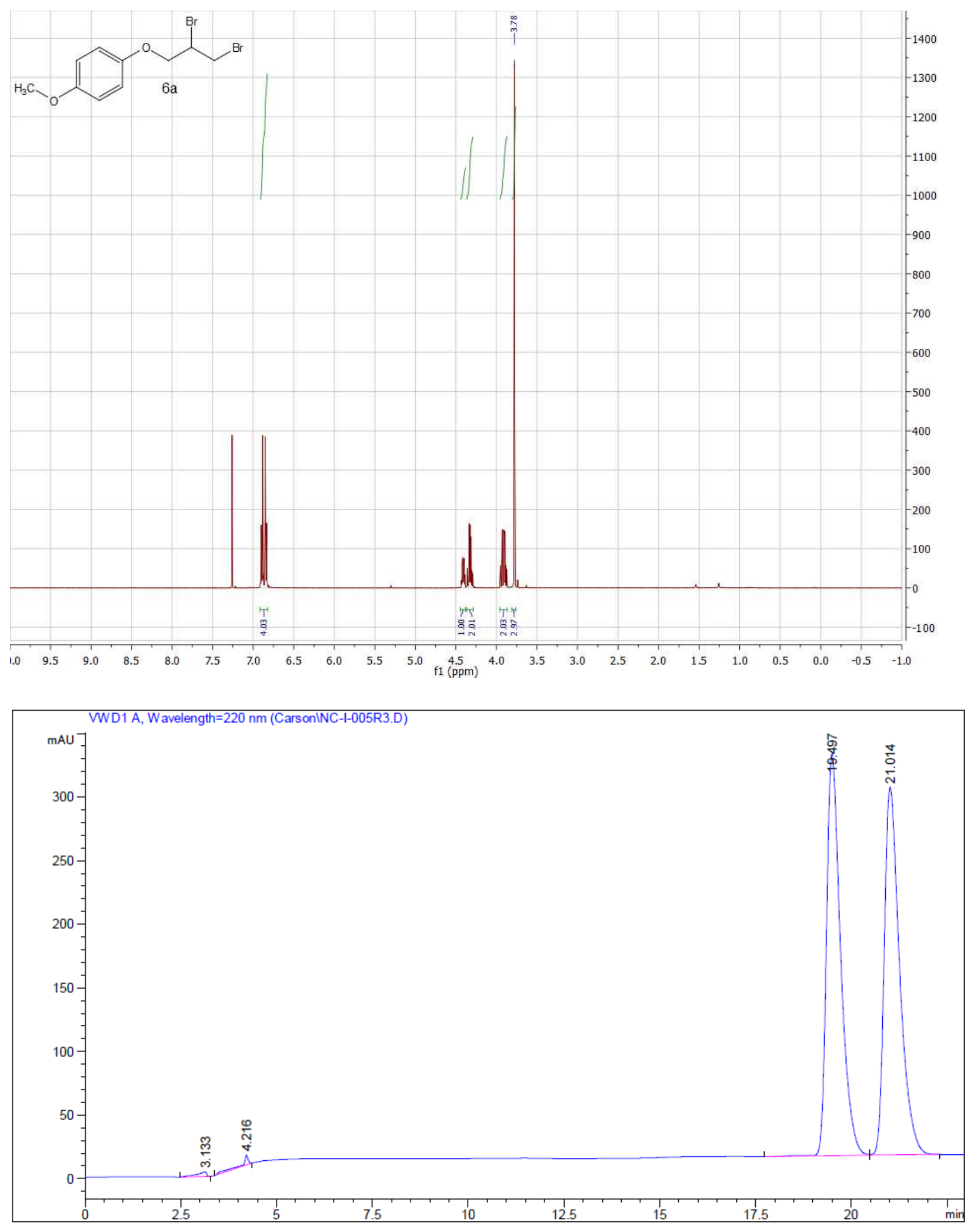
Run 4: Dibromination of 5a with New Catalyst Preparation, $0.25 \mathrm{mmol}, 28 \mathrm{~h}^{\dagger}$

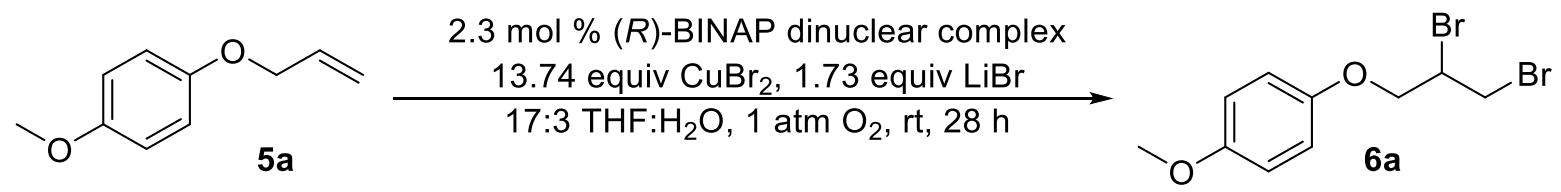

Run 3 was replicated, with the exception of using 4a prepared by the new method described above. Dibromide 6a was produced as a white solid, $69 \mathrm{mg}(0.21 \mathrm{mmol}, \mathbf{8 5 \%}$ yield). This was found to be racemic by CSP-HPLC. ${ }^{1} \mathrm{H}$ NMR spectroscopic data were identical to those of the racemic standard.

Data for 6a:

HPLC: $\quad t_{\mathrm{R}} 19.0 \mathrm{~min}(50.0 \%) ; t_{\mathrm{R}} 21.0 \min (50.0 \%)\left(\mathrm{OJ}-\mathrm{H}\right.$, hexane $/ i-\mathrm{PrOH}=95.5: 4.5,1.0 \mathrm{~mL} \min ^{-1}, 220$ $\left.\mathrm{nm}, 20^{\circ} \mathrm{C}\right)$

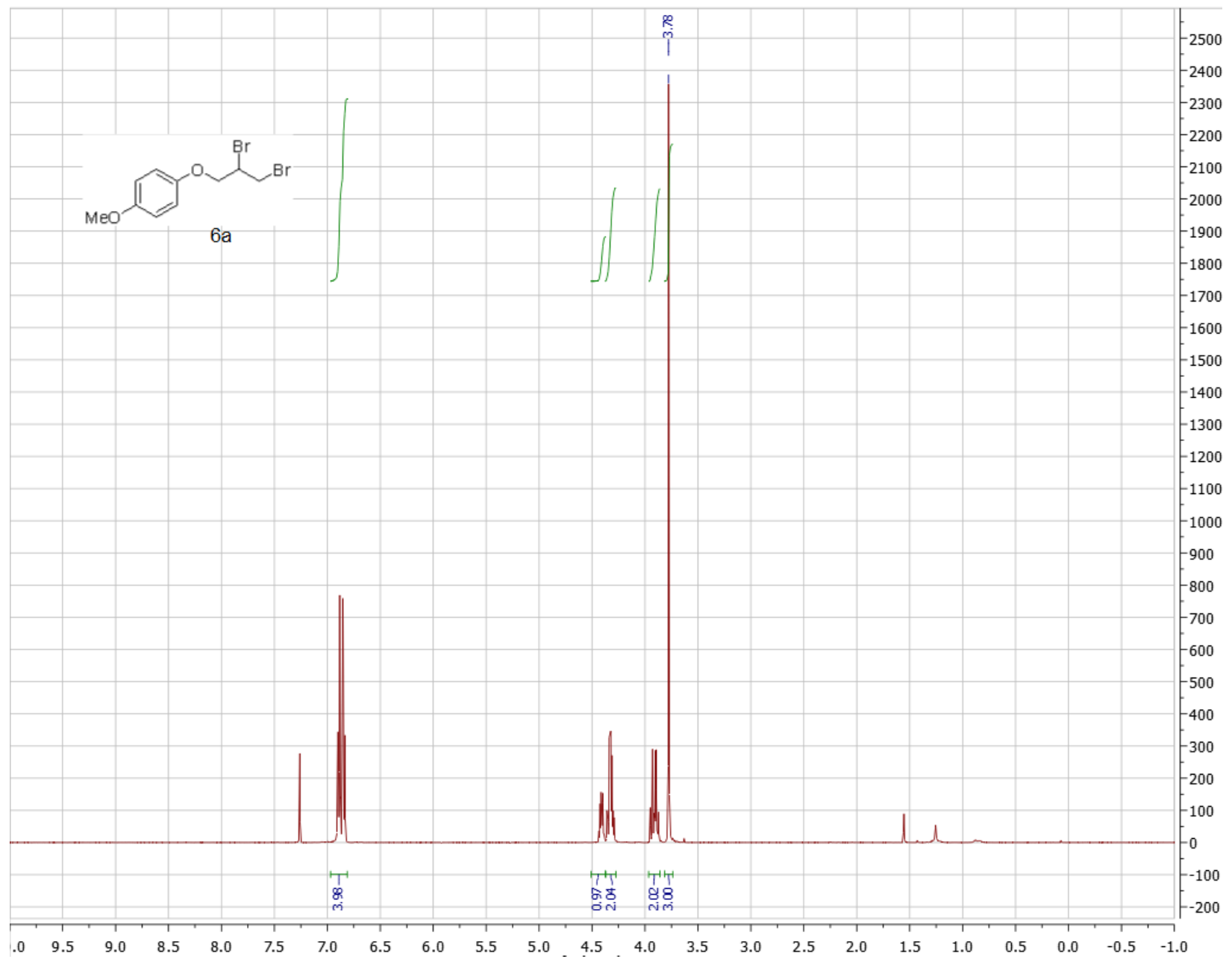




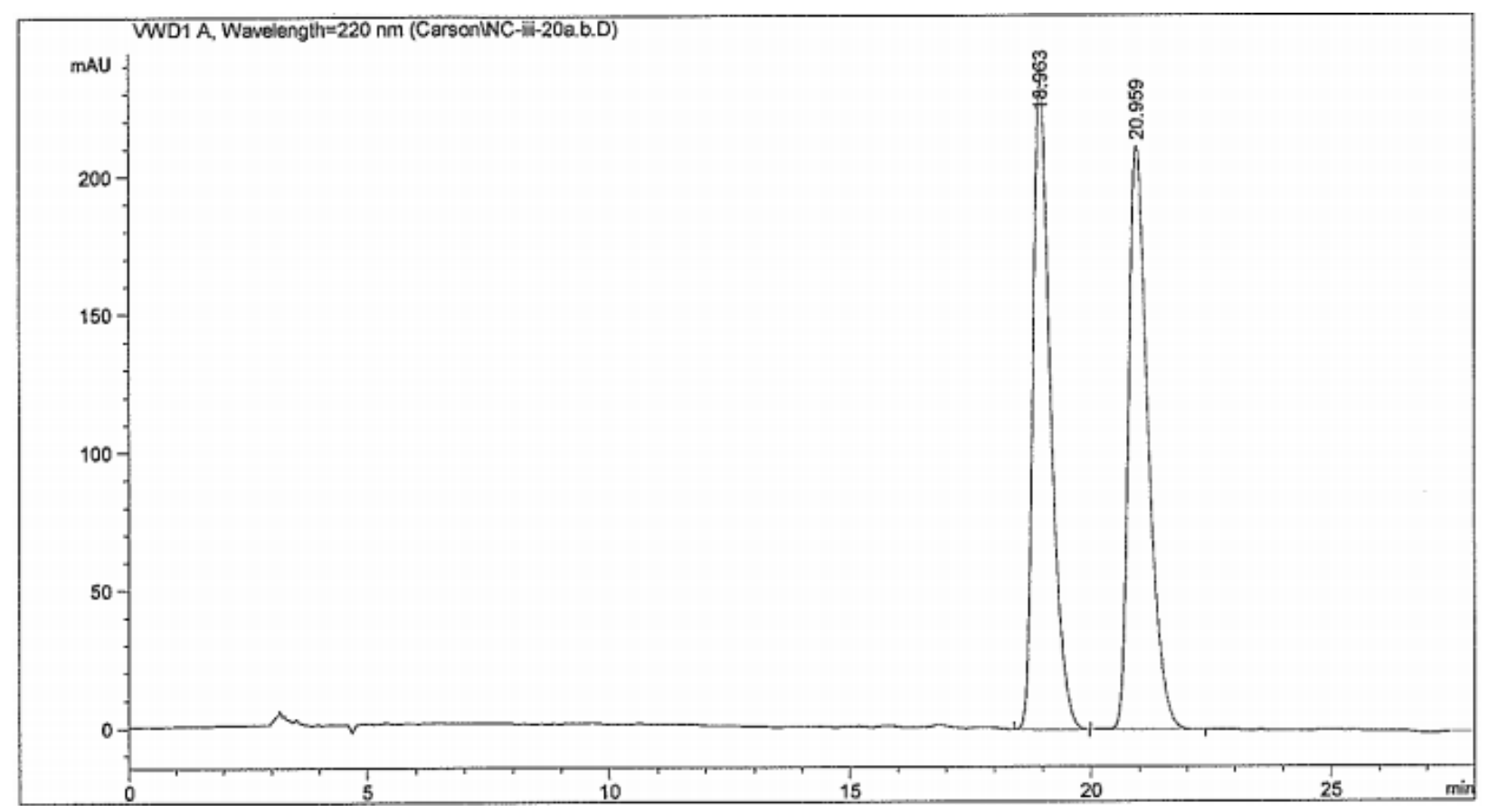




\section{Run 5: Dibromination of 5b, $0.25 \mathrm{mmol}, 24 \mathrm{~h}$}

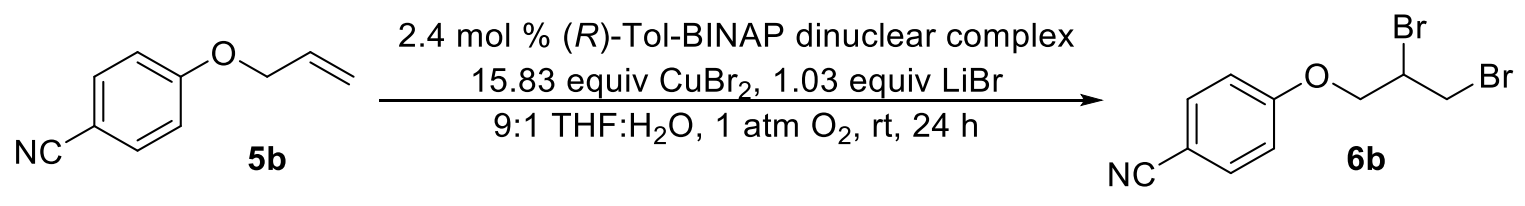

A $25-\mathrm{mL}$, round-bottomed flask containing a Teflon-coated, magnetic stir bar was charged with freshly-prepared $\mathbf{4 b}(8.1 \mathrm{mg}, 6.0 \mu \mathrm{mol}, 2.4 \mathrm{~mol} \%)$, weighed out in a glove box. The system was sealed under $\mathrm{Ar}$, and removed from the glove box, then an Ar line inserted, and 9:1 THF/ $\mathrm{H}_{2} \mathrm{O}(1.0 \mathrm{~mL})$ injected. Then, copper(II) bromide (884 mg, $3.96 \mathrm{mmol}, 15.83$ equiv, $2.0 \mathrm{M}$ ) was added in one portion to form a deep green solution, followed by lithium bromide $(22 \mathrm{mg}, 0.26 \mathrm{mmol}, 1.03$ equiv, $0.13 \mathrm{M})$ in one portion, which gave slight yellowing of the solution. The atmosphere was replaced with $1 \mathrm{~atm} \mathrm{O}_{2}$ by sparging, and the reaction run under $1 \mathrm{~atm} \mathrm{O}_{2}$ from an oxygen manifold. $\mathbf{5 b}(40 \mathrm{mg}, 0.25 \mathrm{mmol})$ was then added to the mixture, washing with 9:1 THF/ $\mathrm{H}_{2} \mathrm{O}(1.0 \mathrm{~mL})$. The mixture was left to stir at rt, monitoring periodically by TLC. Completion was reached at $24 \mathrm{~h}$, and the mixture was extracted with $\mathrm{CH}_{2} \mathrm{Cl}_{2}(3 \times 20 \mathrm{~mL})$, then dried over $\mathrm{MgSO}_{4}$. Hexane $(5 \mathrm{~mL})$ was added, and the precipitated yellow Pd complex removed by filtration, washing with hexane $(3 \times 5 \mathrm{~mL})$. Solvent was removed in vacuo, then the mixture purified via column chromatography using 9:1 hexane/ethyl acetate as eluent, to yield dibromide $\mathbf{6 b}$ as a white solid, $68 \mathrm{mg}(0.21 \mathrm{mmol}, \mathbf{8 5} \%$ yield$)$. This was found to be racemic by CSP-HPLC. ${ }^{1} \mathrm{H}$ NMR spectroscopic data were identical to those of the racemic standard.

\section{Data for $\mathbf{6 b}$ :}

HPLC: $t_{\mathrm{R}} 30.3 \min (49.8 \%) ; t_{\mathrm{R}} 32.6 \min (50.2 \%)\left(\mathrm{IB}-3\right.$, hexane $/ i-\mathrm{PrOH}=92: 8,1.0 \mathrm{~mL} \mathrm{~min}{ }^{-1}$, $\left.220 \mathrm{~nm}, 20^{\circ} \mathrm{C}\right)$ 

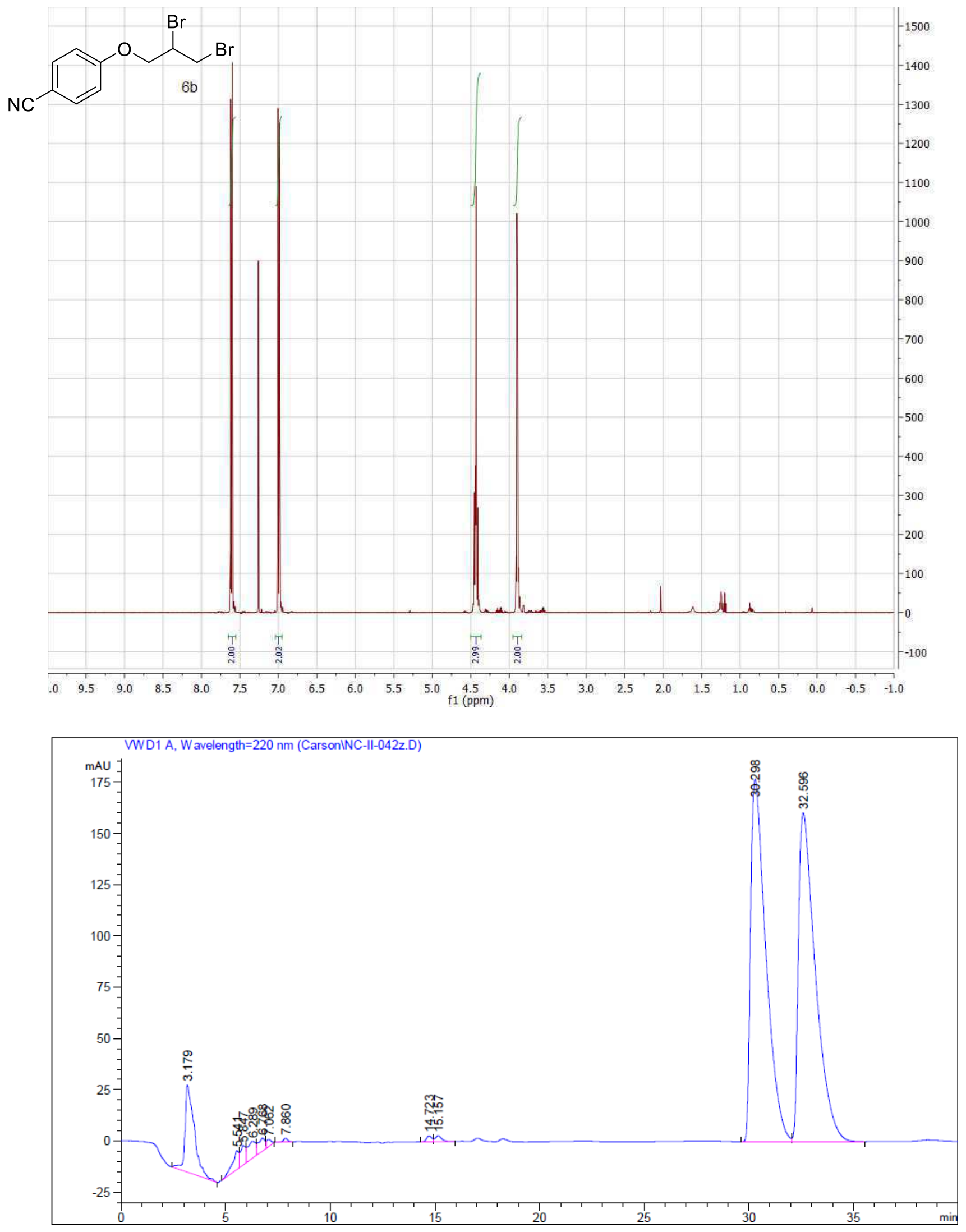


\section{Run 6: Dibromination of $5 \mathrm{c}, 0.25 \mathrm{mmol}, 24 \mathrm{~h}$}

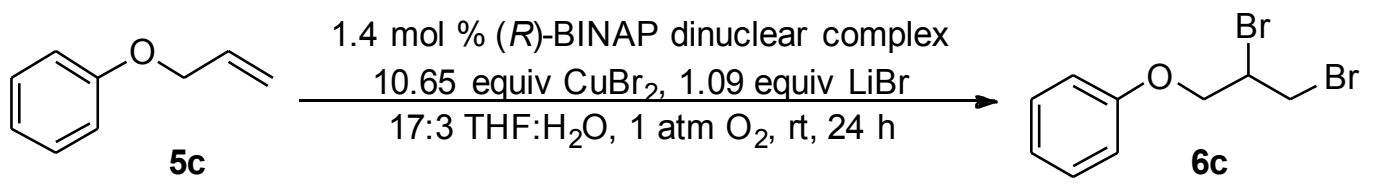

A 25-mL, round-bottomed flask containing a Teflon-coated, magnetic stir bar was charged with 4a, freshly prepared according to the new method described above (4.4 mg, $3.4 \mu \mathrm{mol}, 1.4 \mathrm{~mol} \%$ ), and weighed out in a glove box. The system was sealed under Ar, and removed from the glove box, then an Ar line inserted, and 17:3 THF/ $\mathrm{H}_{2} \mathrm{O}(1.0 \mathrm{~mL})$ injected. Then, copper(II) bromide (595 mg, $2.66 \mathrm{mmol}$, 10.65 equiv, $2.0 \mathrm{M}$ ) was added in one portion to form a deep green solution, followed by lithium bromide (24 mg, $0.27 \mathrm{mmol}, 1.09$ equiv, $0.20 \mathrm{M}$ ) in one portion, which gave slight yellowing of the solution. The atmosphere was replaced with $1 \mathrm{~atm} \mathrm{O}_{2}$ by sparging, and the reaction run under $1 \mathrm{~atm} \mathrm{O}_{2}$ from an oxygen manifold. 5c (34 mg, $0.25 \mathrm{mmol})$ was then added to the mixture, washing with 17:3 THF/ $\mathrm{H}_{2} \mathrm{O}(0.35 \mathrm{~mL})$. The mixture was left to stir at rt, monitoring periodically by TLC. Completion was reached at $24 \mathrm{~h}$, and the mixture was extracted with $\mathrm{CH}_{2} \mathrm{Cl}_{2}(3 \times 20 \mathrm{~mL})$, then dried over $\mathrm{MgSO}_{4}$. Hexane $(5 \mathrm{~mL})$ was added, and the precipitated orange Pd complex removed by filtration, washing with hexane $(3 \times 5 \mathrm{~mL})$. Solvent was removed in vacuo, then the mixture purified via column chromatography using hexane as eluent, to yield dibromide $\mathbf{6 c}$ as a pale yellow oil, $51 \mathrm{mg}(0.17 \mathrm{mmol}, \mathbf{6 9 \%}$ yield $)$. This was found to be racemic by CSP-SFC. ${ }^{1} \mathrm{H}$ NMR spectroscopic data were identical to those of the racemic standard.

Data for 6c:

SFC: $\quad t_{\mathrm{R}} 6.3 \min (49.9 \%) ; t_{\mathrm{R}} 6.6 \min (50.1 \%)\left(\mathrm{OD}, \mathrm{MeOH}: \mathrm{CO}_{2}=3: 97,2.5 \mathrm{~mL} \mathrm{~min}{ }^{-1}, 220 \mathrm{~nm}, 40\right.$ $\left.{ }^{\circ} \mathrm{C}\right)$ 

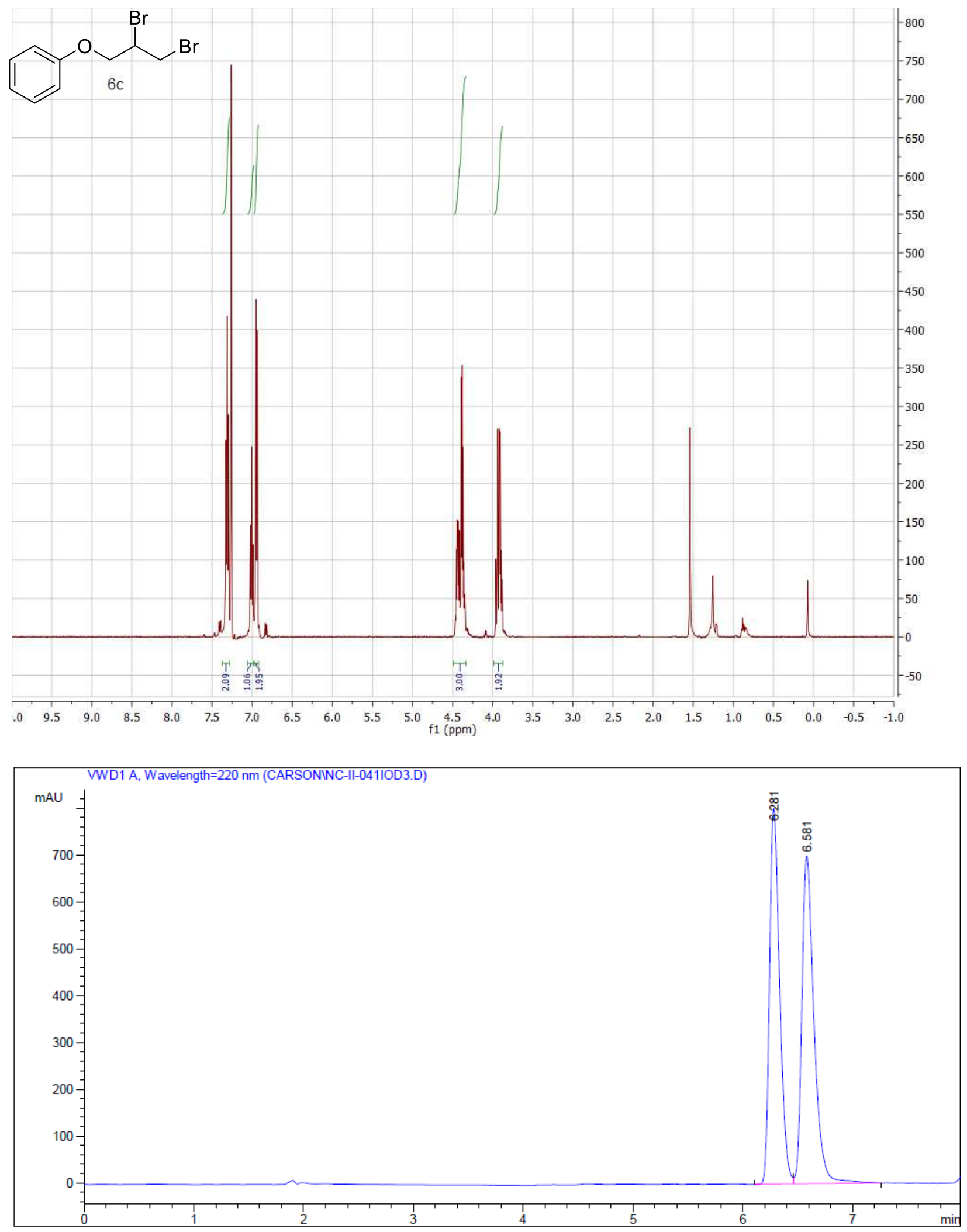


\section{Run 7: Dibromination of 5d, 0.25 mmol, 24 h}

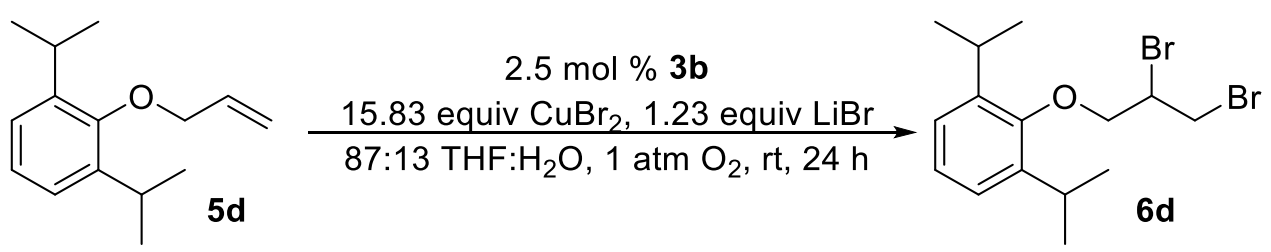

A 25-mL, round-bottomed flask containing a Teflon-coated, magnetic stir bar was charged with freshly-prepared $\mathbf{3 b}(6.2 \mathrm{mg}, 6.3 \mu \mathrm{mol}, 2.5 \mathrm{~mol} \%)$, weighed out in a glove box. The system was sealed under Ar, and removed from the glove box, then an Ar line inserted, and 87:13 THF/ $\mathrm{H}_{2} \mathrm{O}(1.0 \mathrm{~mL})$ injected to give a bright yellow solution. Then, copper(II) bromide (884 mg, $3.96 \mathrm{mmol}, 15.83$ equiv, 2.2 M) was added in one portion to form a deep green solution, followed by lithium bromide $(27 \mathrm{mg}, 0.31$ mmol, 1.23 equiv, $0.17 \mathrm{M}$ ) in one portion, which gave slight yellowing of the solution. The atmosphere was replaced with 1 atm $\mathrm{O}_{2}$ by sparging, and the reaction run under 1 atm $\mathrm{O}_{2}$ from an oxygen manifold. 5d $(55 \mathrm{mg}, 0.25 \mathrm{mmol})$ was then added to the mixture, washing with $87: 13 \mathrm{THF} / \mathrm{H}_{2} \mathrm{O}(0.8 \mathrm{~mL})$. The mixture was left to stir at rt, monitoring periodically by TLC. Completion was reached at $24 \mathrm{~h}$, and the mixture was extracted with $\mathrm{CH}_{2} \mathrm{Cl}_{2}(3 \times 20 \mathrm{~mL})$, then dried over $\mathrm{MgSO}_{4}$. Hexane $(5 \mathrm{~mL})$ was added, and the precipitated yellow Pd complex removed by filtration, washing with hexane $(3 \times 5 \mathrm{~mL})$. Solvent was removed in vacuo, then the mixture purified via column chromatography using 99:1 hexane/ethyl acetate as eluent, to yield dibromide $\mathbf{6 d}$ as colorless needles, $85 \mathrm{mg}(0.22 \mathrm{mmol}, \mathbf{9 0} \%$ yield $)$. This was found to be racemic by CSP-HPLC. ${ }^{1} \mathrm{H}$ NMR spectroscopic data were identical to those of the racemic standard.

\section{Data for 6d:}

HPLC: $\quad t_{\mathrm{R}} 12.8 \min (49.9 \%) ; t_{\mathrm{R}} 13.6 \min (50.1 \%)\left(\mathrm{IB}-3\right.$, hexane $/ i-\mathrm{PrOH}=99.9: 0.1,1.0 \mathrm{~mL} \mathrm{~min}^{-1}, 220$ $\mathrm{nm}, 20^{\circ} \mathrm{C}$ ) 

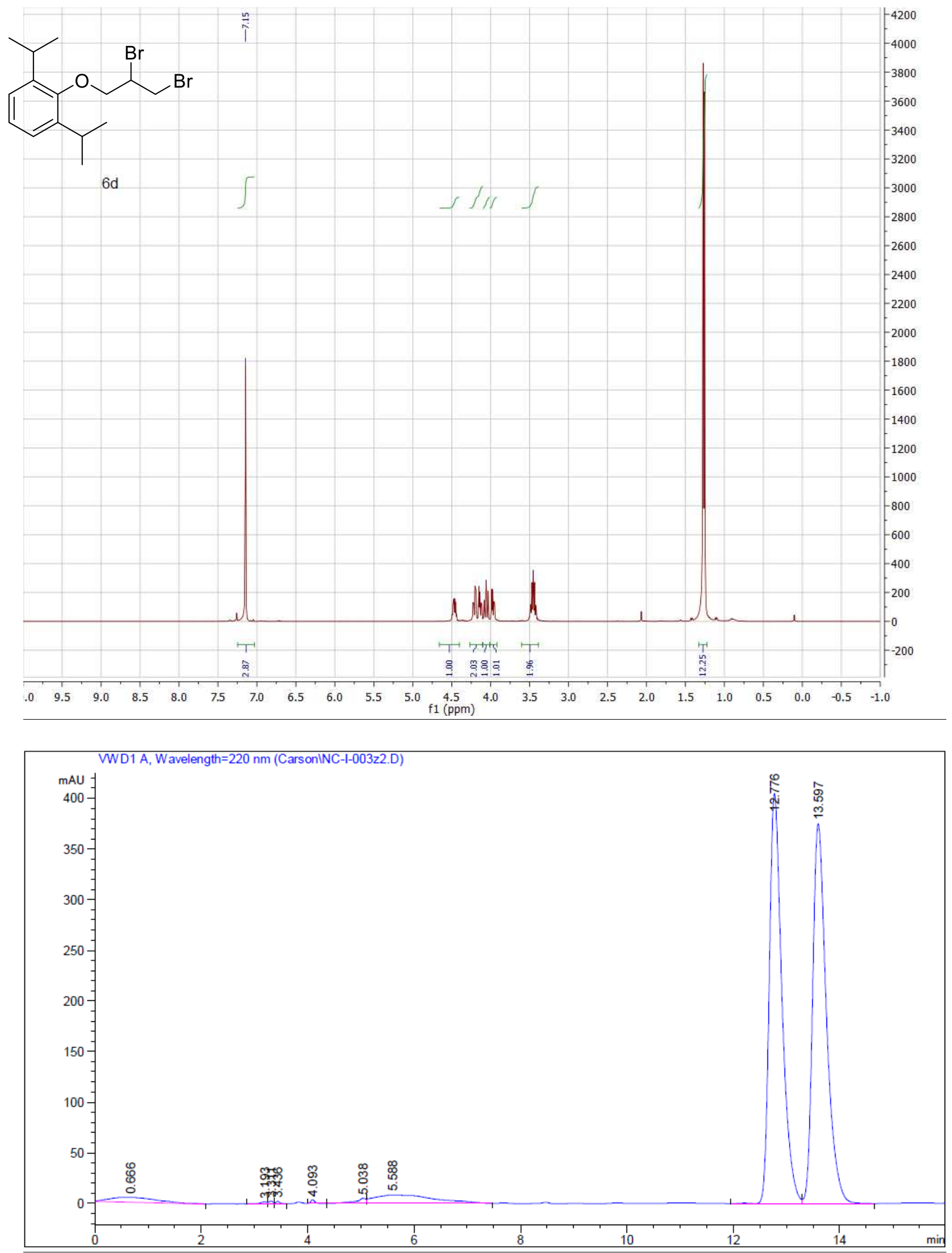


\section{Conditions and Spectra for Chlorohydroxylation Reactions:}

Reactions were run according to Henry's procedures. ${ }^{1}$ The procedures were all reproduced as rigorously as the described procedures allow, other than changing the scale. Reaction progress was monitored by ${ }^{1} \mathrm{H}$ NMR of aliquots. Concentrations are calculated with respect to total solvent volume.

Table S3. Conditions for Chlorohydroxylation Reactions

\begin{tabular}{|c|c|c|c|c|c|c|c|c|c|}
\hline Run & Substrate & $\begin{array}{c}\text { Amount } \\
\text { of } \\
\text { substrate, } \\
\text { mmol }\end{array}$ & $\begin{array}{c}\text { Pd } \\
\text { complex, } \\
\text { mol \%o }\end{array}$ & $\begin{array}{c}\mathbf{C u C l}_{2} \\
\text { equiv }\end{array}$ & $\begin{array}{c}\mathbf{C u C l}_{2} \\
\text { molarity, } \\
\mathbf{M}\end{array}$ & $\begin{array}{c}\text { LiCl } \\
\text { equiv }\end{array}$ & $\begin{array}{c}\text { LiCl } \\
\text { molarity, } \\
\mathbf{M}\end{array}$ & $\begin{array}{c}\mathrm{THF} / \mathrm{H}_{2} \mathrm{O} \\
\text { Time, } \\
\text { days }\end{array}$ \\
\hline $\mathbf{8}$ & $\mathbf{2 a}$ & 0.5 & 2.5 & 20.00 & 4.0 & 1.00 & 0.20 & $2: 1$ & 14 \\
\hline $\mathbf{9}$ & $\mathbf{2 a}$ & 0.5 & 1.2 & 14.59 & 1.8 & 2.43 & 0.30 & $92: 8$ & 5 \\
\hline $\mathbf{1 0}$ & $\mathbf{2 b}$ & 1.0 & 1.0 & 8.00 & 8.0 & 0.40 & 0.40 & $2: 1$ & 10 \\
\hline $\mathbf{1 1}$ & $\mathbf{2 c}$ & 0.5 & 1.5 & 13.38 & 2.9 & 0.92 & 0.20 & $90: 10$ & 10 \\
\hline
\end{tabular}

Run 8: Chlorohydroxylation of $5 \mathrm{c}$ with Catalyst 3a, $0.50 \mathrm{mmol}, 14$ days

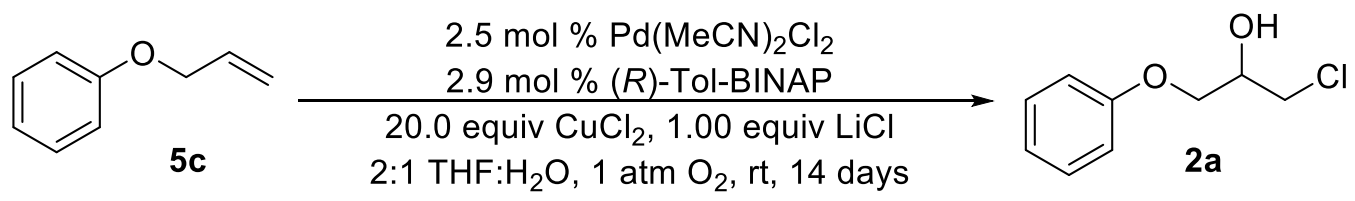

In a glove box, a 10-mL, two-necked flask containing a Teflon-coated, magnetic stir bar was charged with bis(acetonitrile)dichloropalladium(II) $(3.2 \mathrm{mg}, 12 \mu \mathrm{mol}, 2.5 \mathrm{~mol} \%)$. The flask was sealed under Ar and removed from the glove box. An Ar line was inserted, and anhydrous THF (1.0 mL) was injected. $(R)$-Tol-BINAP $(9.5 \mathrm{mg}, 14 \mu \mathrm{mol}, 2.9 \mathrm{~mol} \%$ ) was added to form a yellow solution, which was stirred at $\mathrm{rt}$ for $30 \mathrm{~min}$. Then, a separate $10-\mathrm{mL}$, round-bottomed flask containing a Teflon-coated, magnetic stir bar was charged with anhydrous copper(II) chloride (1.344 g, 10.0 mmol, 20.0 equiv) and lithium chloride (21 mg, $0.5 \mathrm{mmol}, 1.00$ equiv), and 45:55 THF/ $\mathrm{H}_{2} \mathrm{O}(0.5 \mathrm{~mL})$ was added to form a green solution. The catalyst solution was cannulated into the second flask, washing with 45:55 THF/ $\mathrm{H}_{2} \mathrm{O}(0.5$ $\mathrm{mL}$ ). The atmosphere was replaced with 1 atm $\mathrm{O}_{2}$ by sparging, and the reaction run under 1 atm $\mathrm{O}_{2}$ from an oxygen manifold. 5c (67 mg, $0.5 \mathrm{mmol})$ was then added to the mixture, washing with 45:55 THF/ $\mathrm{H}_{2} \mathrm{O}$ $(0.5 \mathrm{~mL})$. The eventual solvent mixture was $2: 1 \mathrm{THF} / \mathrm{H}_{2} \mathrm{O}$. The mixture was left to stir at $\mathrm{rt}$, monitoring periodically by NMR spectroscopy of aliquots. Despite low conversion, the reaction was stopped after 14 days. $\mathrm{H}_{2} \mathrm{O}(10 \mathrm{~mL})$ was added, and the mixture was extracted with $\mathrm{CH}_{2} \mathrm{Cl}_{2}(3 \times 10 \mathrm{~mL})$, then dried over $\mathrm{CaCl}_{2}$. Solvent was removed in vacuo, then the mixture purified via column chromatography using a gradient from 93:7 to 85:15 hexane/ethyl acetate as eluent, to yield chlorohydrin 2a as a pale yellow oil, 
$14 \mathrm{mg}\left(0.075 \mathrm{mmol}, \mathbf{1 5 \%}\right.$ yield). This was found to be racemic by CSP-SFC. ${ }^{1} \mathrm{H}$ NMR spectroscopic data were identical to those of the racemic standard.

\section{Data for 2a:}

SFC: $\quad t_{\mathrm{R}} 9.8 \min (49.5 \%) ; t_{\mathrm{R}} 11.7 \mathrm{~min}(50.5 \%)\left(\mathrm{OD}, \mathrm{MeOH}: \mathrm{CO}_{2}=1: 99\right.$ for $10 \min$ then $10: 90$ for 6

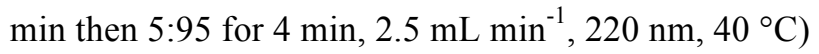




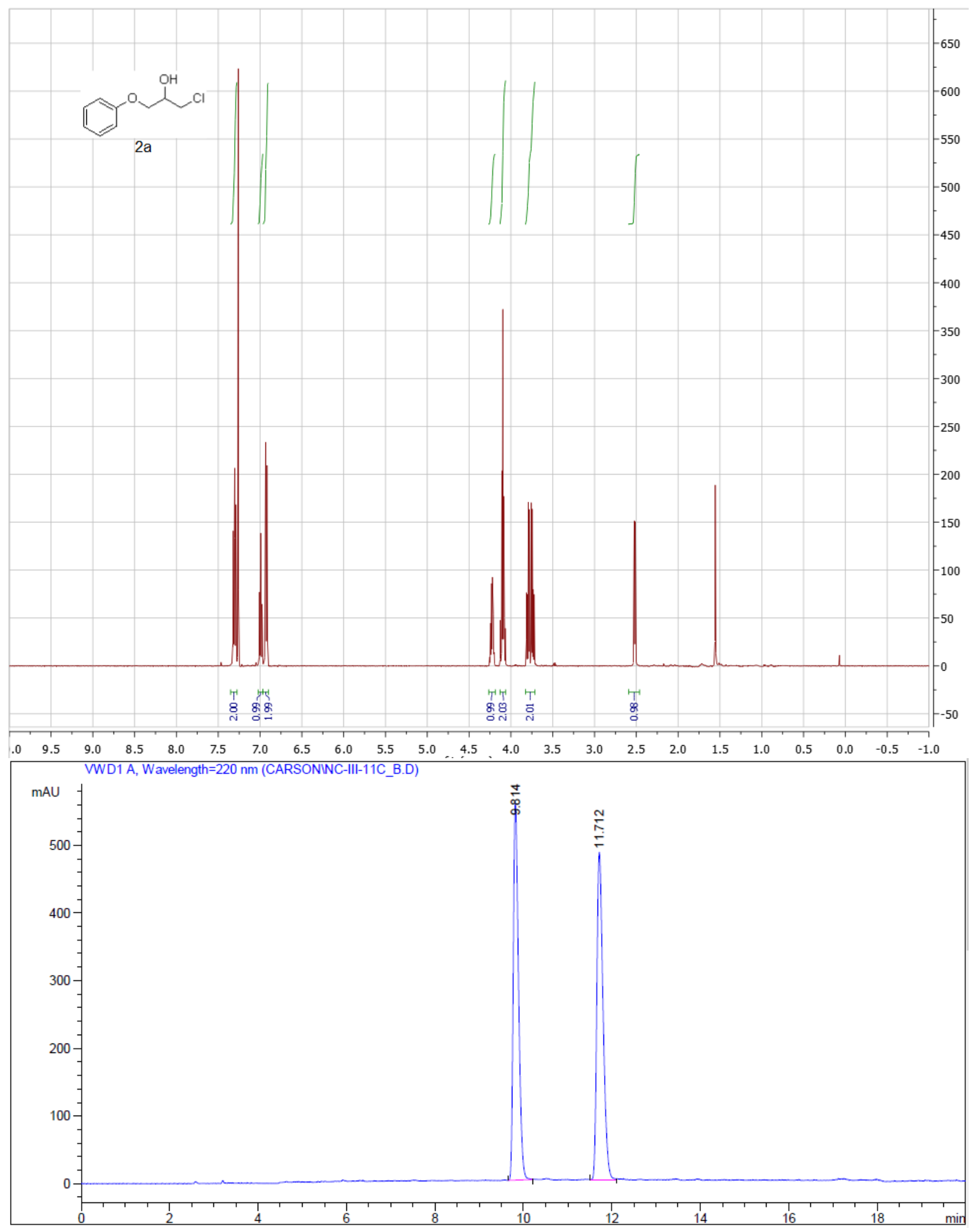




\section{Run 9: Chlorohydroxylation of $5 \mathrm{c}$ with Catalyst 4a, $0.50 \mathrm{mmol}, 5$ days}

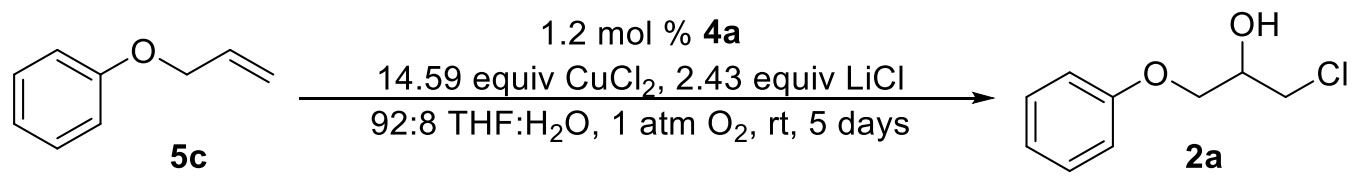

In a glove box, a 10-mL, two-necked flask containing a Teflon-coated, magnetic stir bar was charged with 4a, freshly prepared according to Henry's method (7.9 mg, $6.1 \mu \mathrm{mol}, 1.2 \mathrm{~mol} \%)$. The flask was sealed under Ar and removed from the glove box. An Ar line was inserted, and anhydrous THF (1.0 $\mathrm{mL}$ ) was injected. Anhydrous copper(II) chloride (981 mg, $7.30 \mathrm{mmol}, 14.59$ equiv) and lithium chloride (52 mg, $1.21 \mathrm{mmol}, 2.43$ equiv) were then added, followed by 89:11 THF/ $\mathrm{H}_{2} \mathrm{O}(2.05 \mathrm{~mL}$ ), to form a green solution. The atmosphere was replaced with $1 \mathrm{~atm} \mathrm{O}_{2}$ by sparging, and the reaction run under 1 atm $\mathrm{O}_{2}$ from an oxygen manifold. 5c $(67 \mathrm{mg}, 0.5 \mathrm{mmol})$ was then added to the mixture, washing with $92: 8$ $\mathrm{THF} / \mathrm{H}_{2} \mathrm{O}(1.0 \mathrm{~mL})$. The eventual solvent mixture was $92: 8 \mathrm{THF} / \mathrm{H}_{2} \mathrm{O}$. The mixture was left to stir at $\mathrm{rt}$, monitoring periodically by NMR spectroscopy of aliquots, and stopped after 5 days. $\mathrm{H}_{2} \mathrm{O}(10 \mathrm{~mL})$ was added, and the mixture was extracted with $\mathrm{CH}_{2} \mathrm{Cl}_{2}(3 \times 10 \mathrm{~mL})$, then dried over $\mathrm{CaCl}_{2}$. Solvent was removed in vacuo, then the mixture purified via column chromatography using a gradient from 93:7 to 85:15 hexane/ethyl acetate as eluent, to yield chlorohydrin 2a as a pale yellow oil, $37 \mathrm{mg}(0.20 \mathrm{mmol}$, $\mathbf{4 0} \%$ yield). This was found to be racemic by CSP-SFC. ${ }^{1} \mathrm{H}$ NMR spectroscopic data were identical to those of the racemic standard.

\section{Data for 2a:}

SFC: $\quad t_{\mathrm{R}} 9.7 \mathrm{~min}(49.5 \%) ; t_{\mathrm{R}} 11.6 \min (50.5 \%)\left(\mathrm{OD}, \mathrm{MeOH}: \mathrm{CO}_{2}=1: 99\right.$ for $10 \mathrm{~min}$ then $10: 90$ for 6

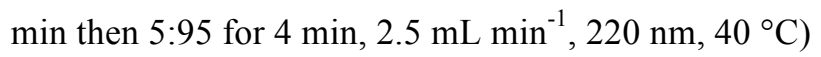



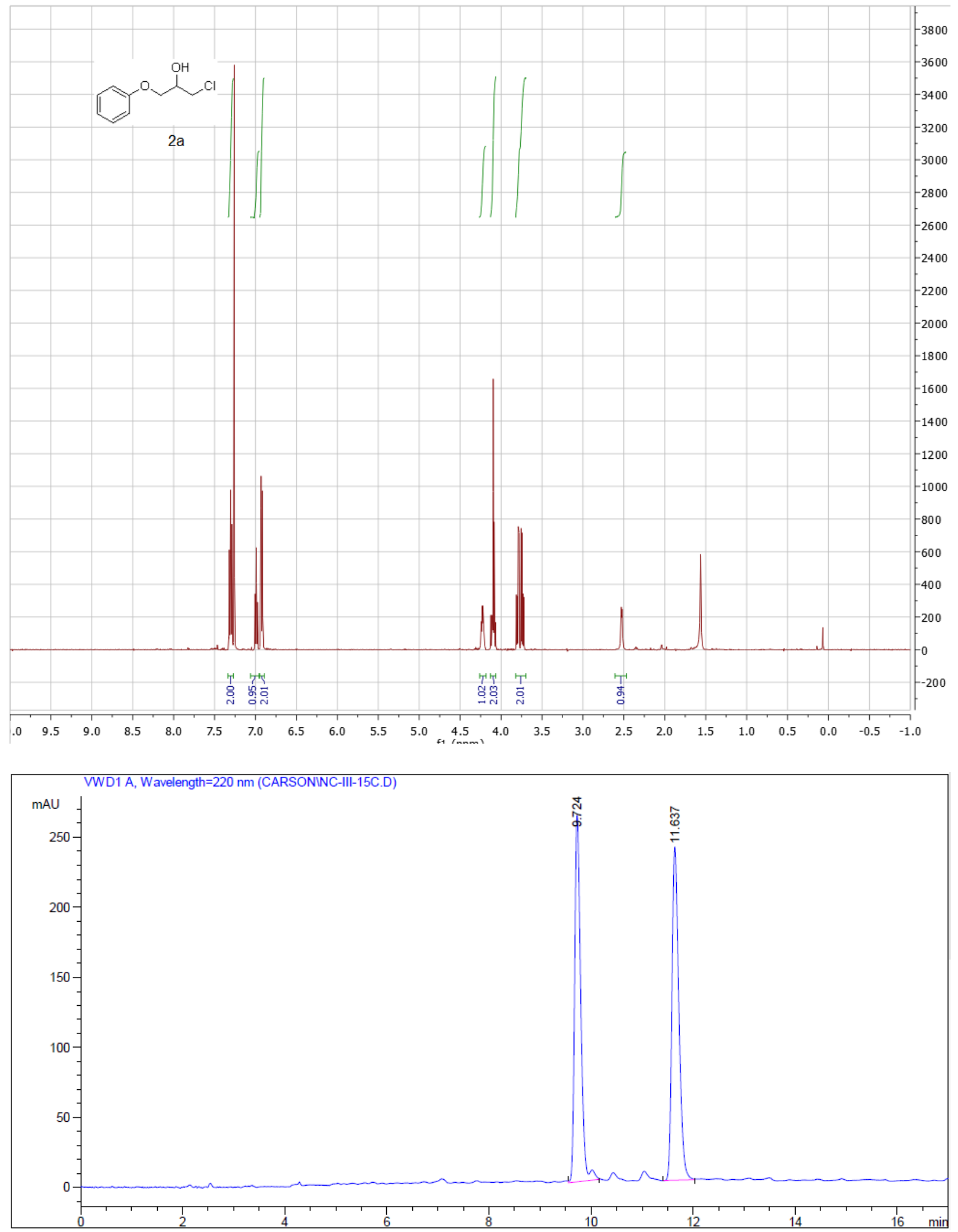


\section{Run 10: Chlorohydroxylation of Methyl Vinyl Ketone with Catalyst 3a, 1.00 mmol, 10 days}

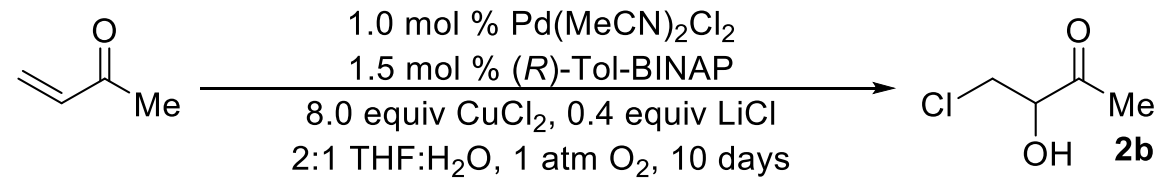

In a glove box, a 10-mL, two-necked flask containing a Teflon-coated, magnetic stir bar was charged with bis(acetonitrile)dichloropalladium(II) $(2.6 \mathrm{mg}, 10 \mu \mathrm{mol}, 1.0 \mathrm{~mol} \%)$. The flask was sealed under Ar and removed from the glove box. An Ar line was inserted, and anhydrous THF (1.0 mL) was injected. $(R)$-Tol-BINAP $(9.9 \mathrm{mg}, 15 \mu \mathrm{mol}, 1.5 \mathrm{~mol} \%$ ) was added to form a yellow solution, which was stirred at $\mathrm{rt}$ for $30 \mathrm{~min}$. Then, a separate $10-\mathrm{mL}$, round-bottomed flask containing a Teflon-coated, magnetic stir bar was charged with anhydrous copper(II) chloride (1.076 g, 8.0 mmol, 8.0 equiv) and lithium chloride (17 mg, $0.4 \mathrm{mmol}, 0.4$ equiv), then 1:2 $\mathrm{THF} / \mathrm{H}_{2} \mathrm{O}(0.3 \mathrm{~mL})$ was added to form a green solution. The catalyst solution was cannulated into the second flask, washing with $1: 2 \mathrm{THF} / \mathrm{H}_{2} \mathrm{O}(0.3$ $\mathrm{mL}$ ). The atmosphere was replaced with 1 atm $\mathrm{O}_{2}$ by sparging, and the reaction run under 1 atm $\mathrm{O}_{2}$ from an oxygen manifold. Methyl vinyl ketone $(70 \mathrm{mg}, 1.0 \mathrm{mmol})$ was then injected into the mixture, washing with 1:2 THF/ $\mathrm{H}_{2} \mathrm{O}(0.4 \mathrm{~mL})$. The eventual solvent mixture was $2: 1 \mathrm{THF} / \mathrm{H}_{2} \mathrm{O}$. The mixture was left to stir at rt, monitoring periodically by NMR spectroscopy of aliquots, and stopped after 10 days. $\mathrm{H}_{2} \mathrm{O}(10$ $\mathrm{mL}$ ) was added, and the mixture was extracted with $\mathrm{CH}_{2} \mathrm{Cl}_{2}(3 \times 10 \mathrm{~mL})$, then dried over $\mathrm{CaCl}_{2}$. Solvent was removed in vacuo, then the mixture purified via column chromatography using a gradient from 95:5 to $80: 20$ hexane/ethyl acetate as eluent, to yield chlorohydrin $\mathbf{2 b}$ as a colorless oil, $40 \mathrm{mg}(0.33 \mathrm{mmol}$, 33\% yield). This was found to be racemic by CSP-GC. ${ }^{1} \mathrm{H}$ NMR spectroscopic data were identical to those of the racemic standard.

Data for $\mathbf{2 b}$ :

GC: $\quad t_{\mathrm{R}} 16.9 \min (50.3 \%) ; t_{\mathrm{R}} 17.7 \min (49.7 \%)\left(\right.$ CycloSil-B, $\left.70{ }^{\circ} \mathrm{C}\right)$ 

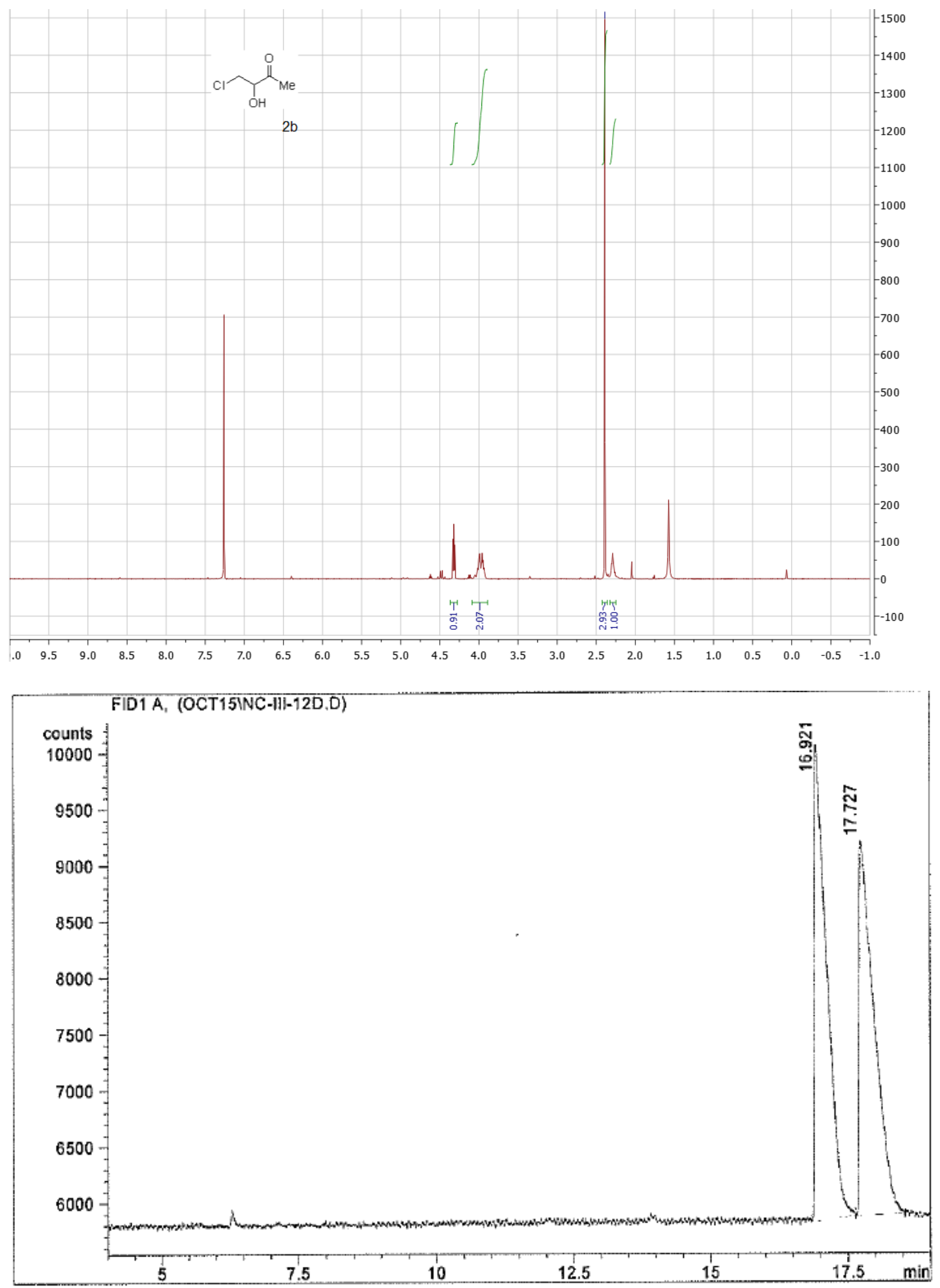


\section{Run 11: Chlorohydroxylation of 5e with Catalyst 4a, $0.50 \mathrm{mmol}, 14$ days}

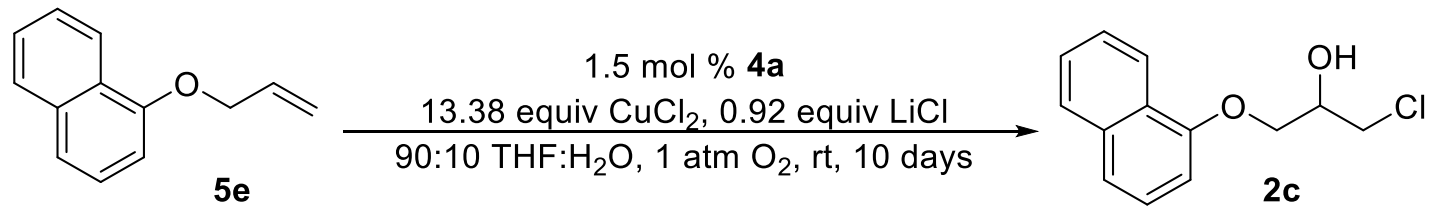

NOTE: The product was mischaracterized in Henry and coworkers' original report. The product there characterized was present in small amounts (roughly 9\%) in the crude reaction mixture and is proposed to be the constitutionally isomeric chlorohydroxylation product.

In a glove box, a 10-mL, two-necked flask containing a Teflon-coated, magnetic stir bar was charged with 4a, freshly prepared according to Henry's method (9.9 mg, $7.7 \mu \mathrm{mol}, 1.5 \mathrm{~mol} \%)$. The flask was sealed under Ar and removed from the glove box. An Ar line was inserted, and anhydrous THF (1.0 $\mathrm{mL}$ ) was injected. Anhydrous copper(II) chloride (900 mg, $6.69 \mathrm{mmol}, 13.38$ equiv) and lithium chloride (20 mg, $0.46 \mathrm{mmol}, 0.92$ equiv) were then added, followed by $82: 18 \mathrm{THF} / \mathrm{H}_{2} \mathrm{O}(0.7 \mathrm{~mL}$ ), to form a green solution. The atmosphere was replaced with $1 \mathrm{~atm} \mathrm{O}_{2}$ by sparging, and the reaction run under 1 atm $\mathrm{O}_{2}$ from an oxygen manifold. 5e (92 $\mathrm{mg}, 0.5 \mathrm{mmol})$ was then added to the mixture, washing with $82: 18$ $\mathrm{THF} / \mathrm{H}_{2} \mathrm{O}(0.6 \mathrm{~mL})$. The eventual solvent mixture was $90: 10 \mathrm{THF} / \mathrm{H}_{2} \mathrm{O}$. The mixture was left to stir at rt, monitoring periodically by NMR spectroscopy of aliquots. Despite incomplete conversion, the reaction was stopped after 10 days. $\mathrm{H}_{2} \mathrm{O}(10 \mathrm{~mL})$ was added, and the mixture was extracted with $\mathrm{CH}_{2} \mathrm{Cl}_{2}(3 \times 10$ $\mathrm{mL}$ ), then dried over $\mathrm{CaCl}_{2}$. Solvent was removed in vacuo, then the mixture purified via column chromatography using a gradient from $90: 10$ to $30: 70$ hexane/ $\mathrm{Et}_{2} \mathrm{O}$ as eluent, to yield firstly recovered 54 mg of pink solid 5e (0.29 mmol, 59\% yield of recovered starting material), and secondly chlorohydrin $2 \mathbf{c}$ as a pale yellow oil, $34 \mathrm{mg}(0.14 \mathrm{mmol}, \mathbf{2 9} \%$ yield $)$. This was found to be racemic by CSP-SFC. ${ }^{1} \mathrm{H}$ NMR spectroscopic data were identical to those of the racemic standard.

\section{Data for $2 \mathrm{c}$ :}

SFC: $t_{\mathrm{R}} 24.6 \min (50.1 \%) ; t_{\mathrm{R}} 32.7 \mathrm{~min}(49.9 \%)\left(\mathrm{OD}, \mathrm{MeOH}: \mathrm{CO}_{2}=10: 90,2.5 \mathrm{~mL} \mathrm{~min}^{-1}, 220 \mathrm{~nm}, 40\right.$ $\left.{ }^{\circ} \mathrm{C}\right)$ 

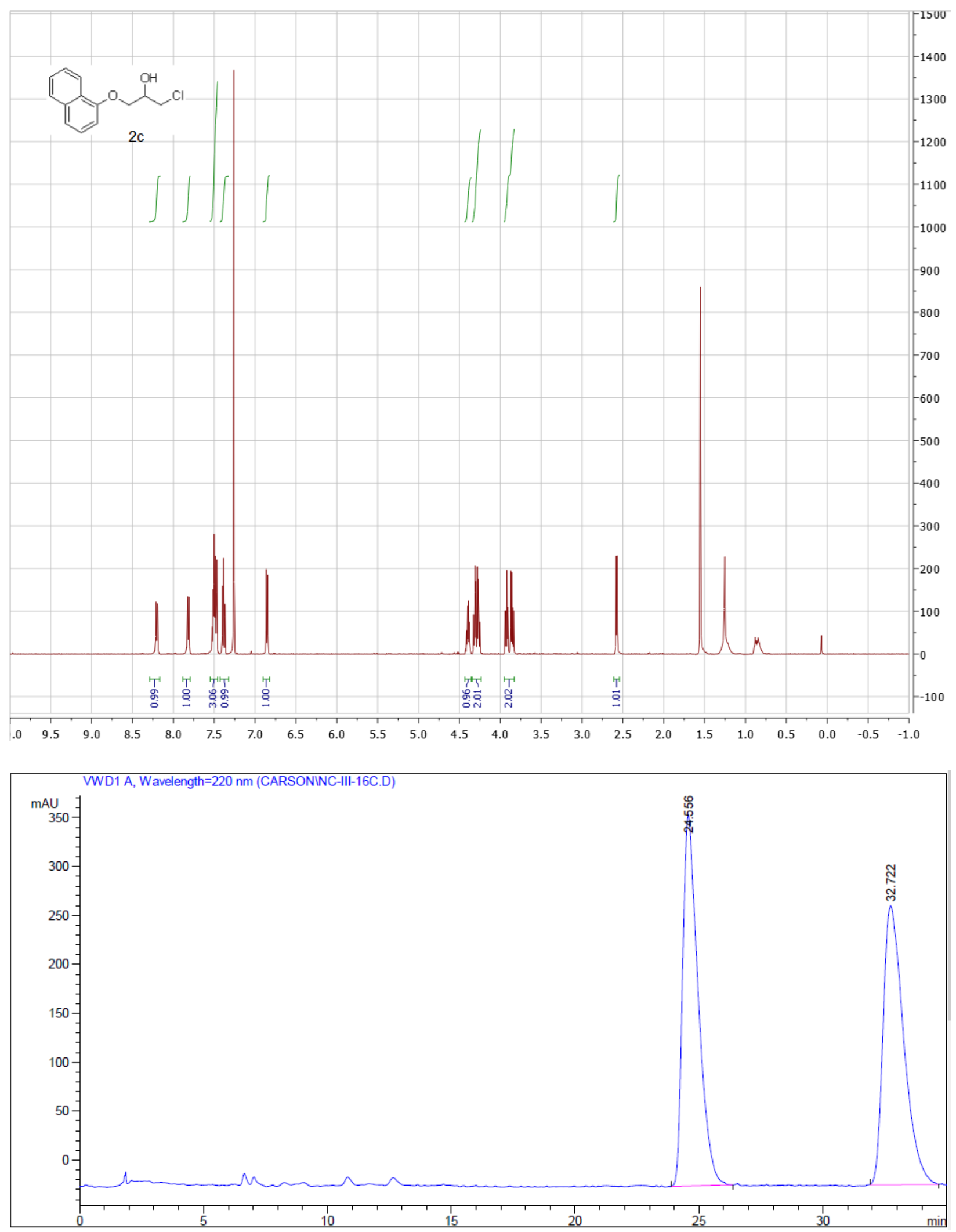


\section{Chiral Shift Reagent NMR Experiment}

$\mathrm{CDCl}_{3}$ was purified by passing through a dry neutral $\mathrm{Al}_{2} \mathrm{O}_{3}$ column $(70 \times 7 \mathrm{~mm}$ diameter $)$. $\mathrm{Eu}(\mathrm{hfc})_{3}(448 \mathrm{mg}, 375 \mu \mathrm{mol})$ was dissolved in $2.0 \mathrm{~mL}$ of purified $\mathrm{CDCl}_{3}$, and the bright yellow solution filtered through a Celite column $(25 \times 6 \mathrm{~mm}$ diameter $)$ with a slight positive Ar flow. Dibromide $5 \mathbf{a}(40.5$ $\mathrm{mg}, 125 \mu \mathrm{mol})$ was dissolved in purified $\mathrm{CDCl}_{3}(0.5 \mathrm{~mL})$ in an NMR tube. The $\mathrm{Eu}(\mathrm{hfc})_{3}$ solution was added portionwise to the solution of $\mathbf{5 a}$, and ${ }^{1} \mathrm{H}$ NMR spectra (400 $\mathrm{MHz}$ ) were taken after each addition (see page $\mathrm{S} 48$ ). The spectrum corresponding to 4 equiv $\mathrm{Eu}(\mathrm{hfc})_{3}$ was taken in $\mathrm{CDCl}_{3}(0.8 \mathrm{~mL})$, containing 5a $(23.8 \mathrm{mg}, 70 \mu \mathrm{mol})$ and $\mathrm{Eu}(\mathrm{hfc})_{3}(334 \mathrm{mg}, 280 \mu \mathrm{mol})$. The processing parameters for this spectrum were:

$\begin{array}{ll}\text { Spectrometer frequency } & 400 \mathrm{MHz} \\ \text { Delay time } & 10 \mathrm{~s} \\ \text { Number of scans } & 16\end{array}$

\section{References}

(1) El-Qisairi, A. K.; Qaseer, H. A.; Katsigras, G.; Lorenzi, P.; Trivedi, U.; Tracz, S.; Hartman, A.; Miller, J. A.; Henry, P. M. Org. Lett. 2003, 5, 439-441.

(2) Armarego, W. L. F.; Chai, C. L. L. Purification of Laboratory Chemicals, Sixth Ed.; Butterworth-Heinemann, 2009.

(3) El-Qisairi, A. K.; Qaseer, H. A.; Henry, P. M. J. Organomet. Chem. 2002, 656, 168-176.

(4) Marcinkiewicz, S.; Green, J.; Mamalis, P. Tetrahedron 1961, 14, 208-222.

(5) Nesper, R.; Pregosin, P. S.; Püntener, K.; Wörle, M. Helv. Chim. Acta 1993, 76, 2239-2249.

(6) El-Qisairi, A.; Henry, P. M. J. Organomet. Chem. 2000, 603, 50-60.

(7) Braddock, D. C.; Cansell, G.; Hermitage, S. A. Synlett 2004, 461-464.

(8) Martínez, F.; Del Campo, C.; Sinisterra, J. V.; Llama, E. F. Tetrahedron Asymmetry 2000, 11, $4651-4660$.

(9) Limnios, D.; Kokotos, C. G. J. Org. Chem. 2014, 79, 4270-4276. 


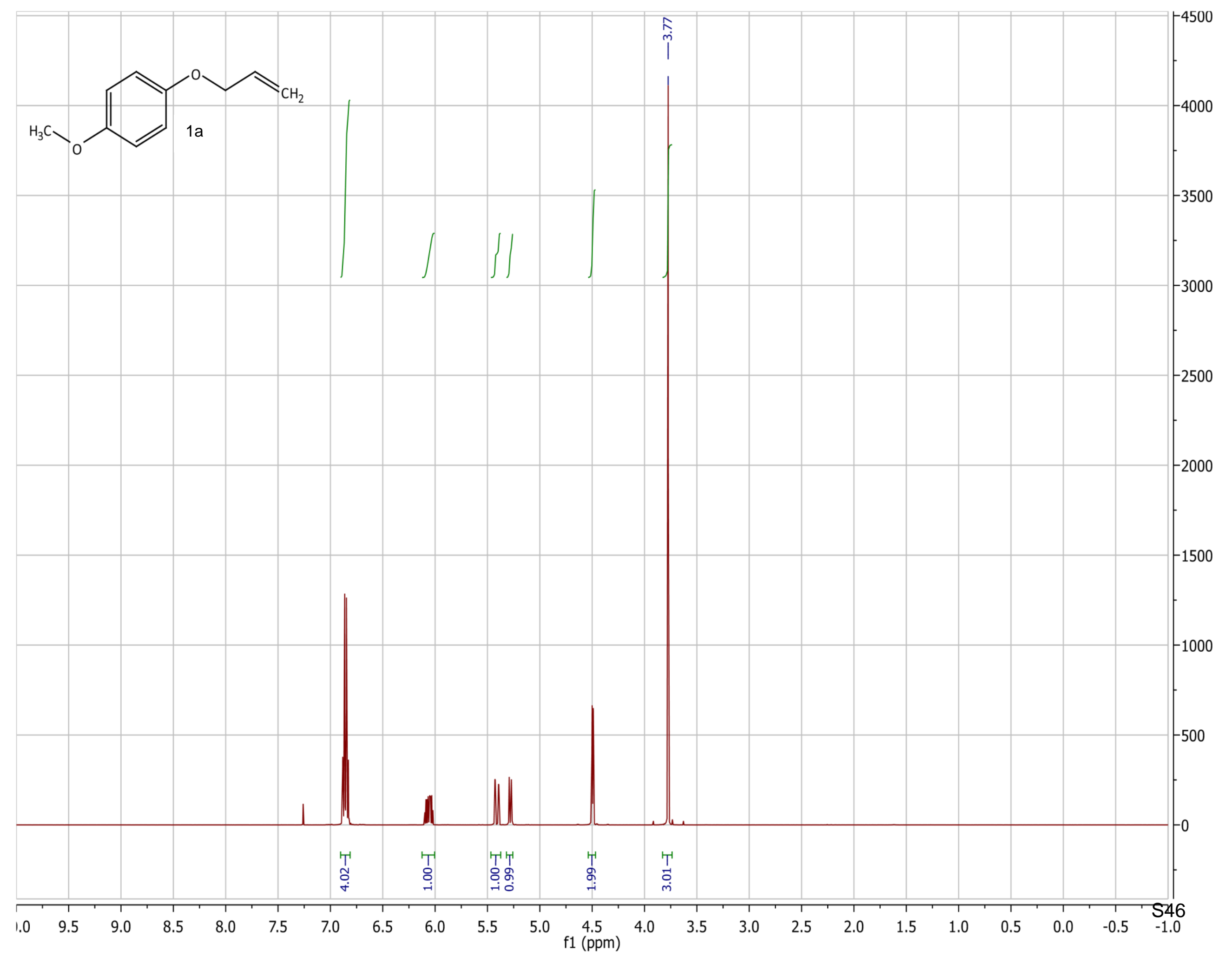




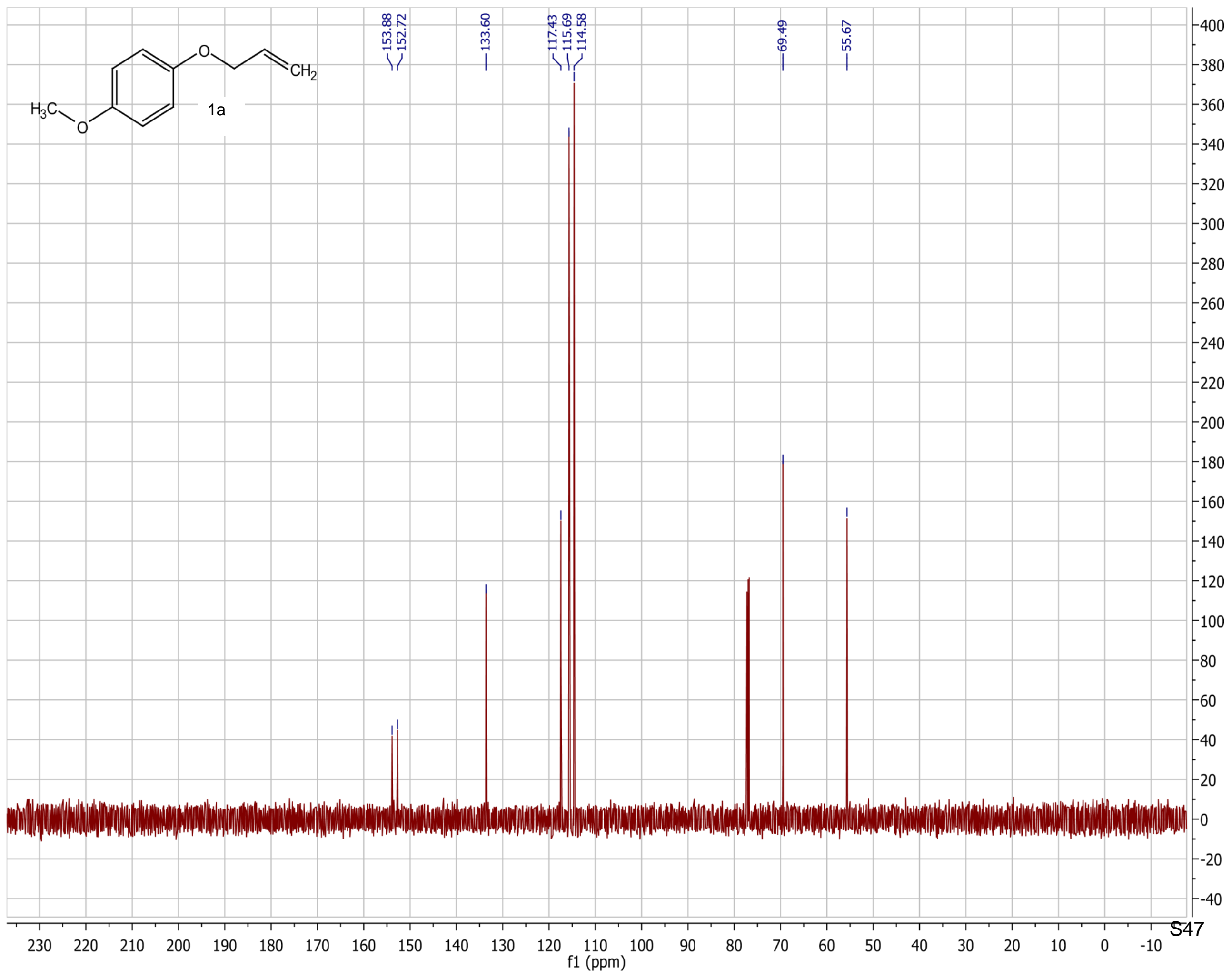




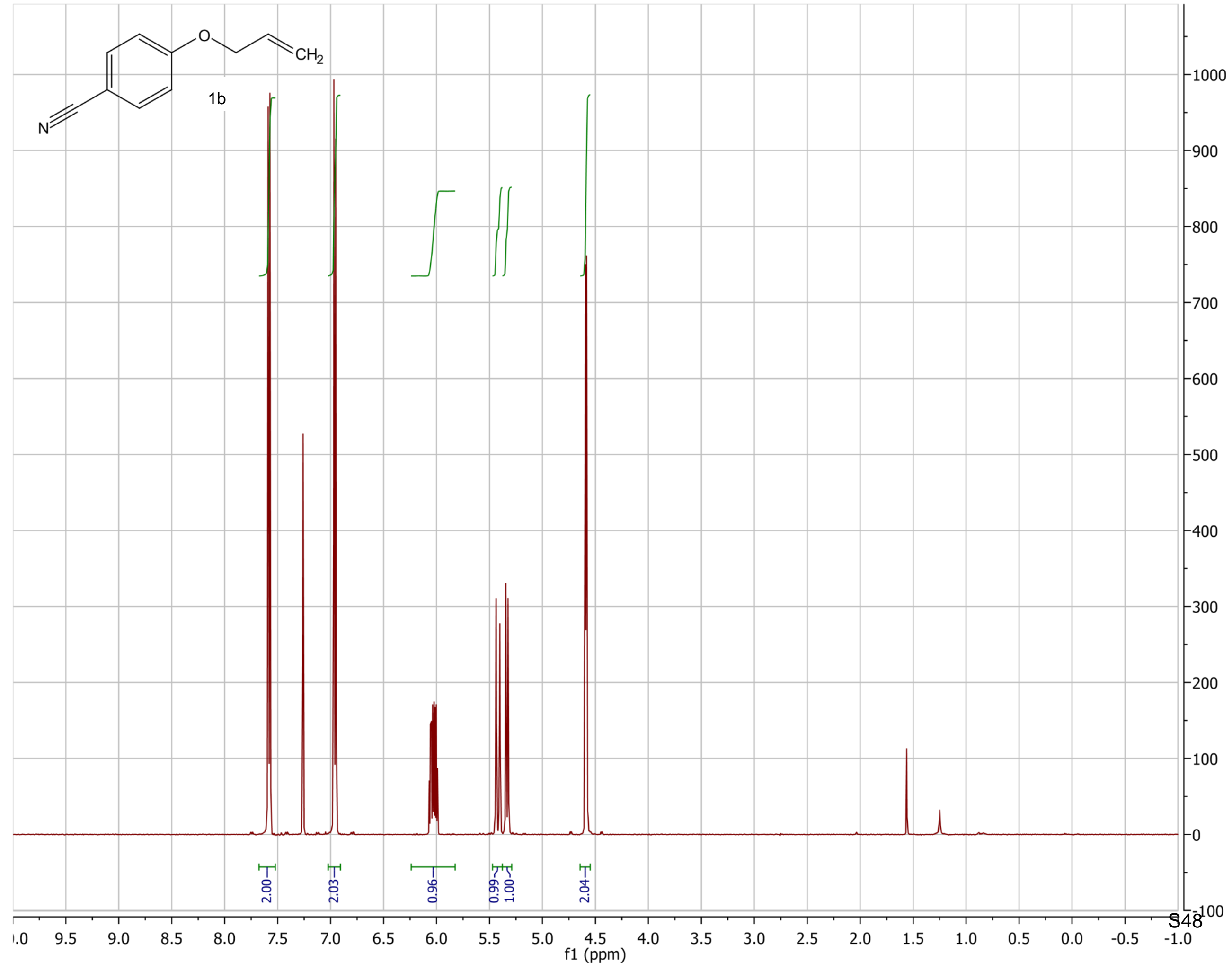




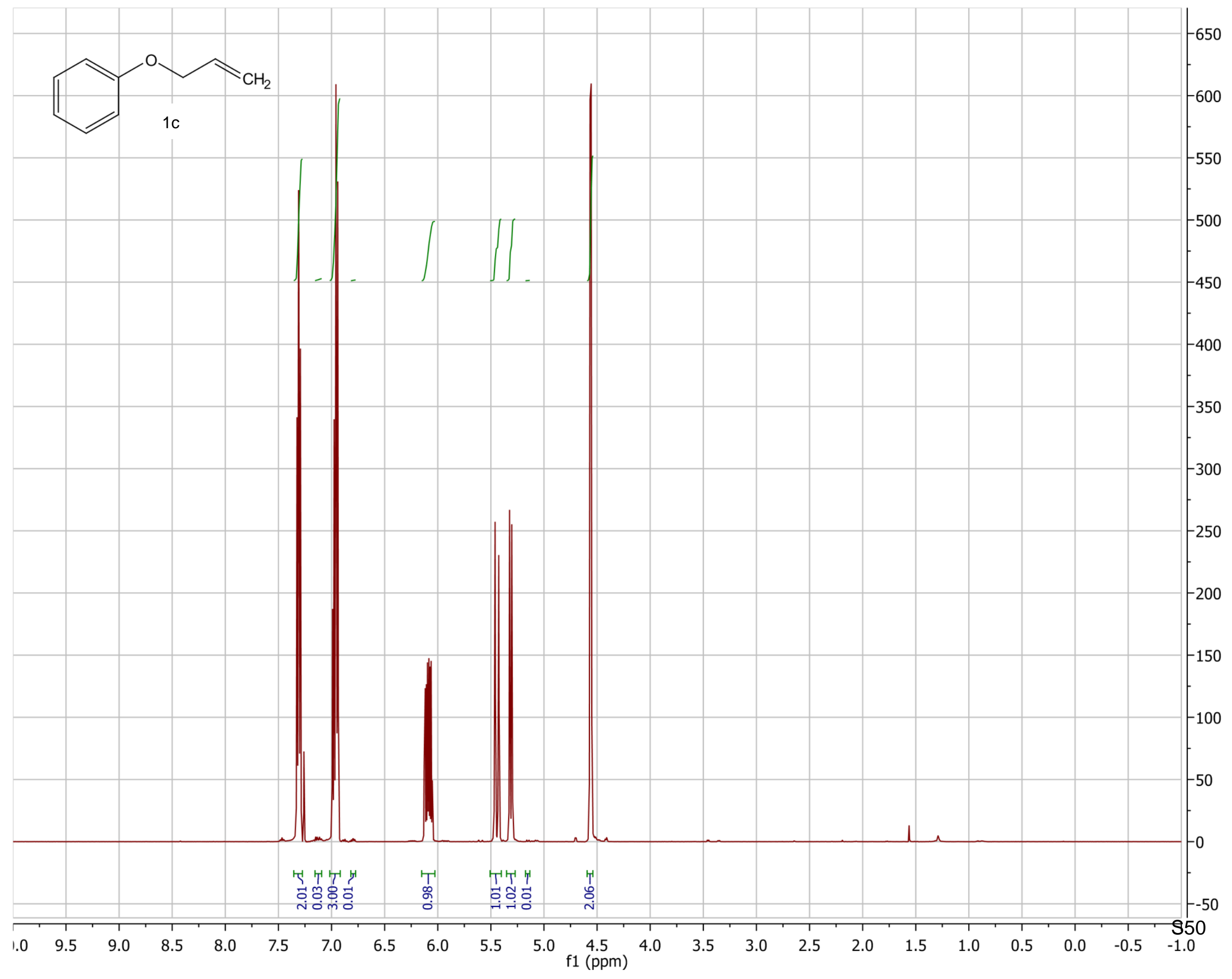





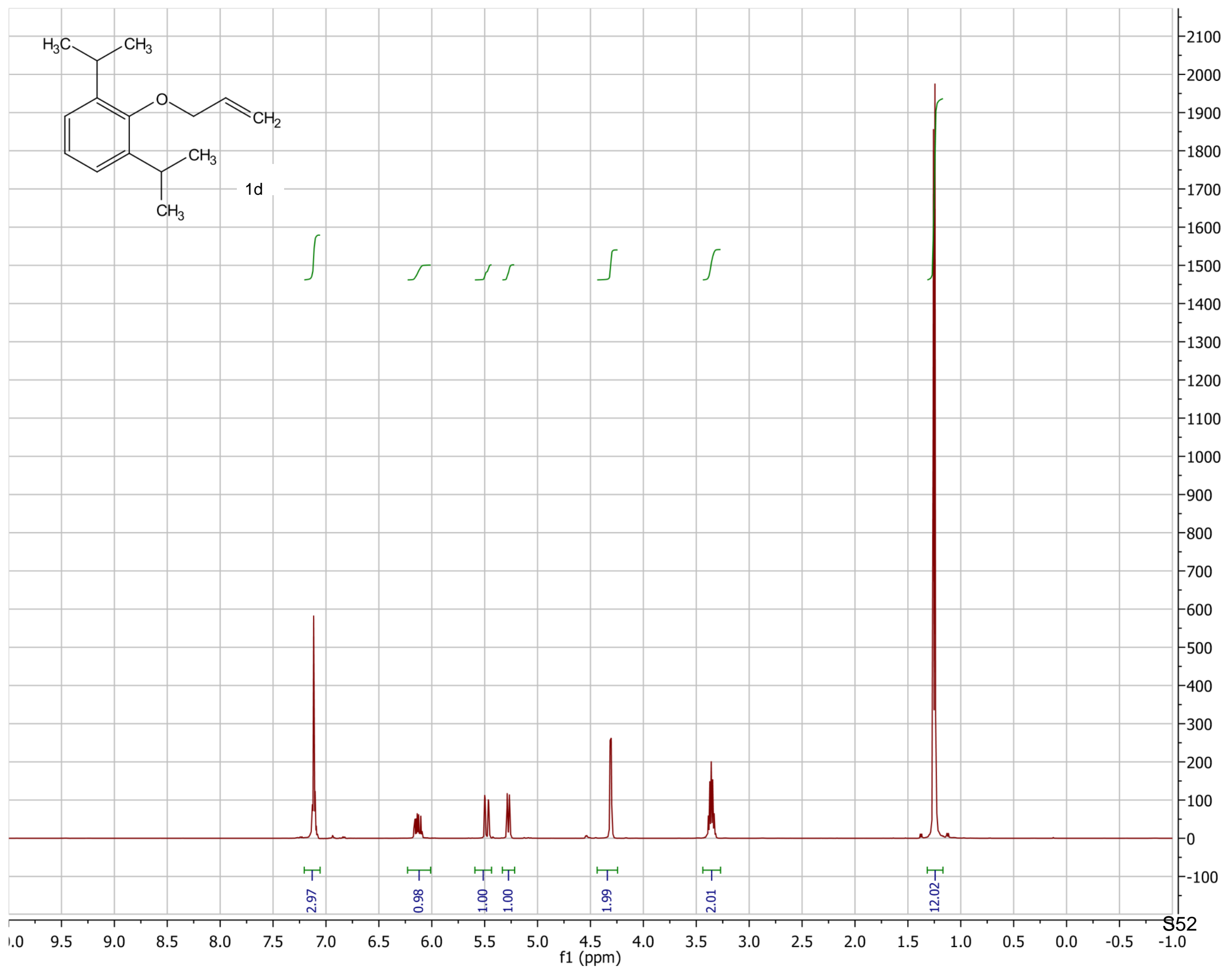




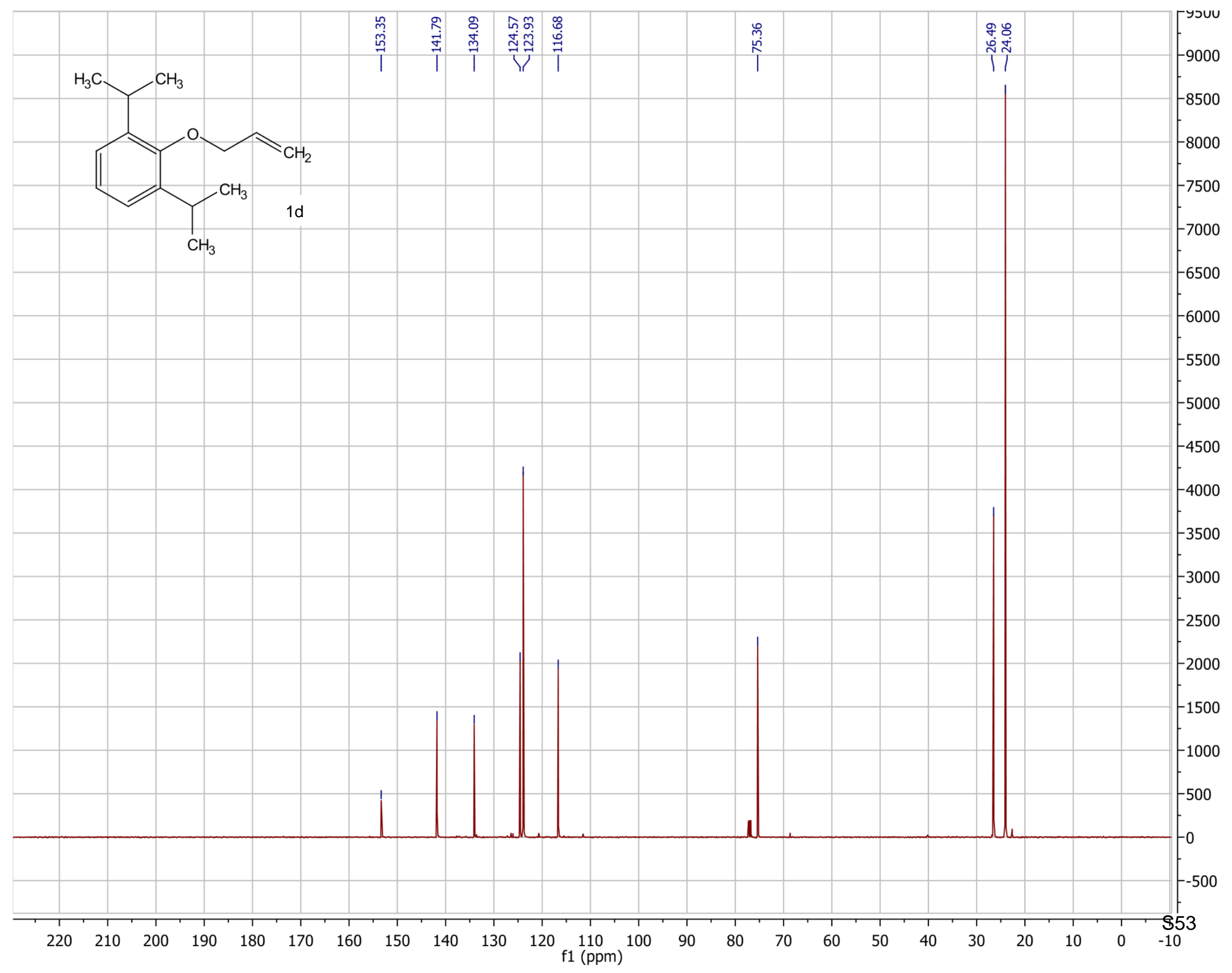




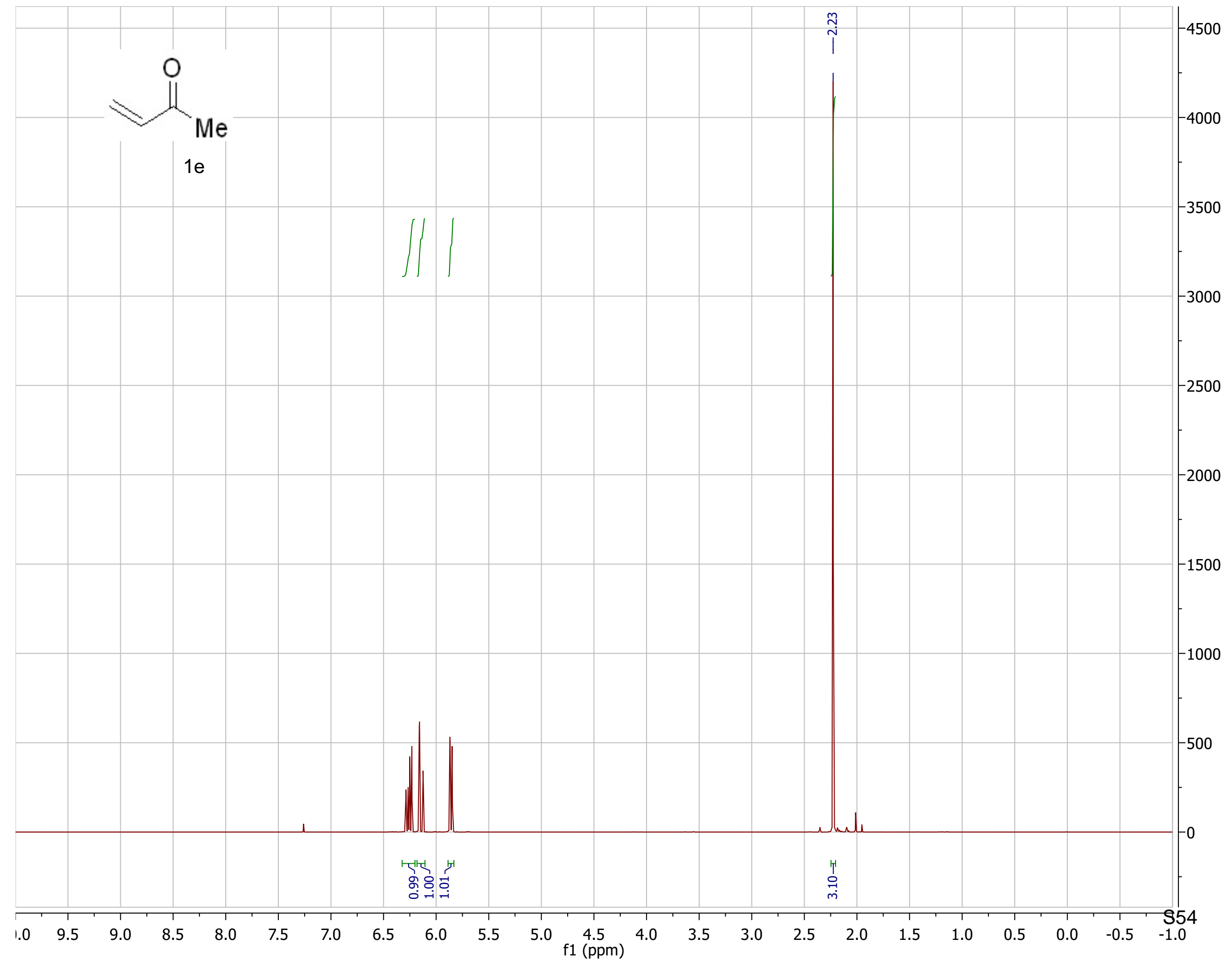




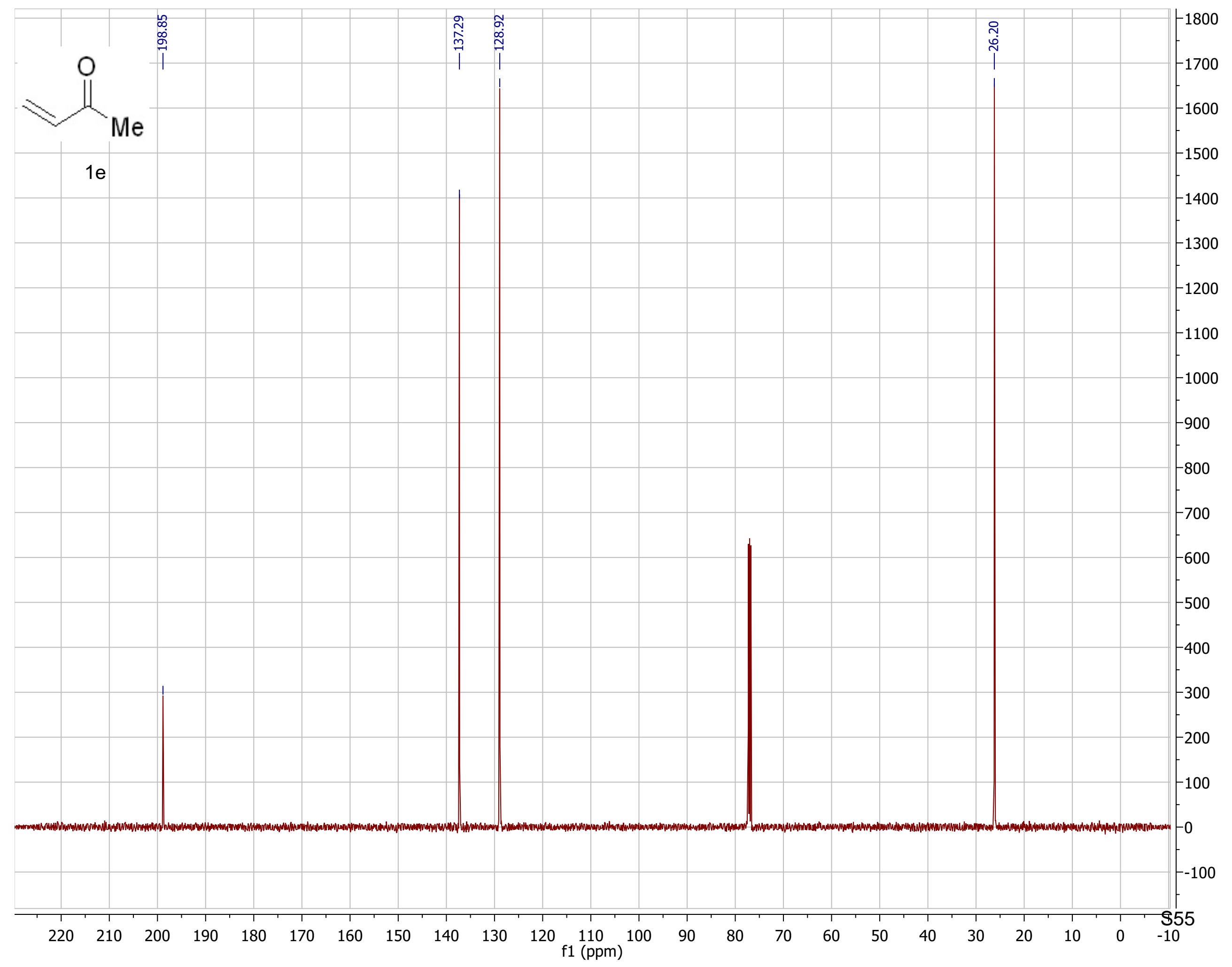




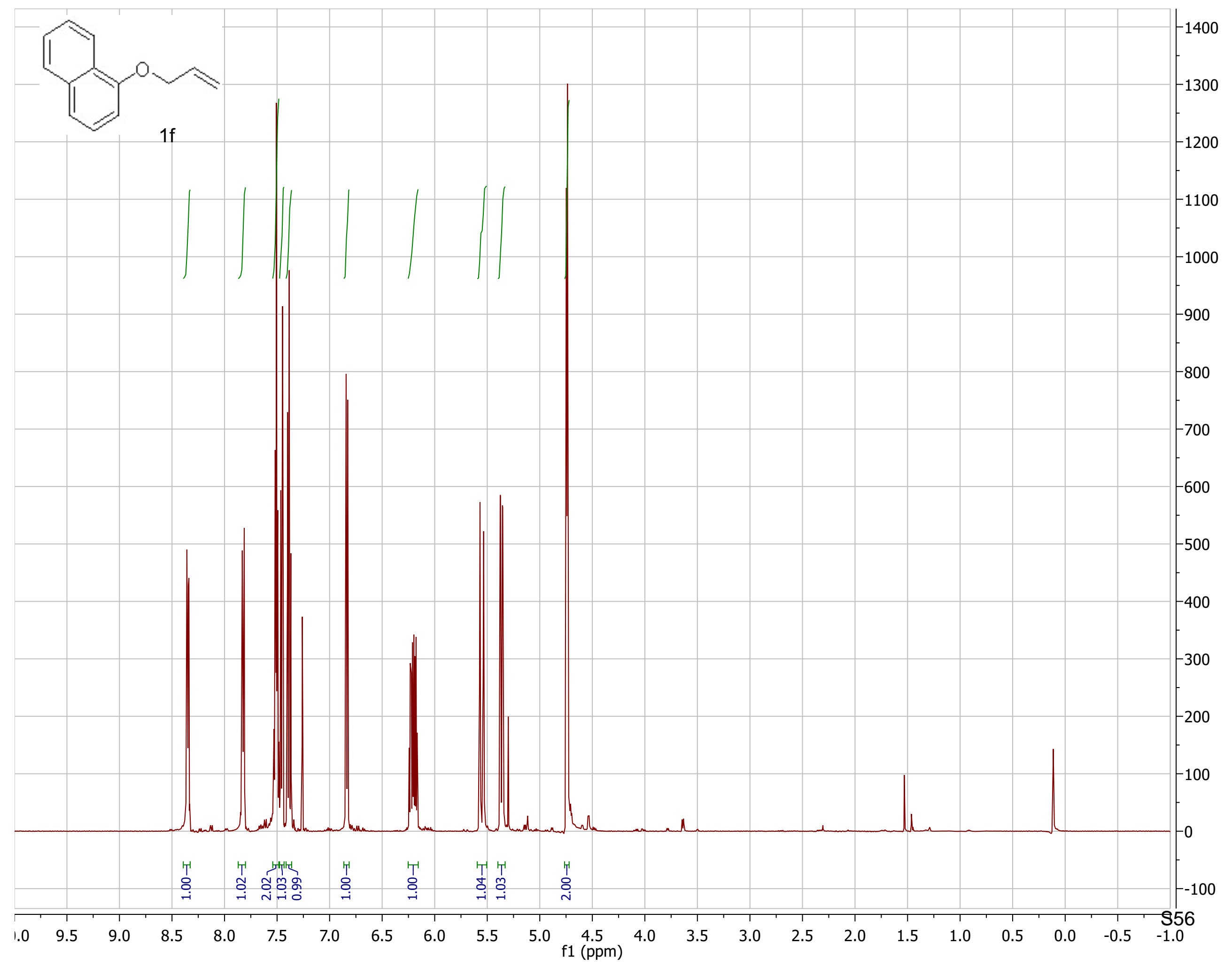



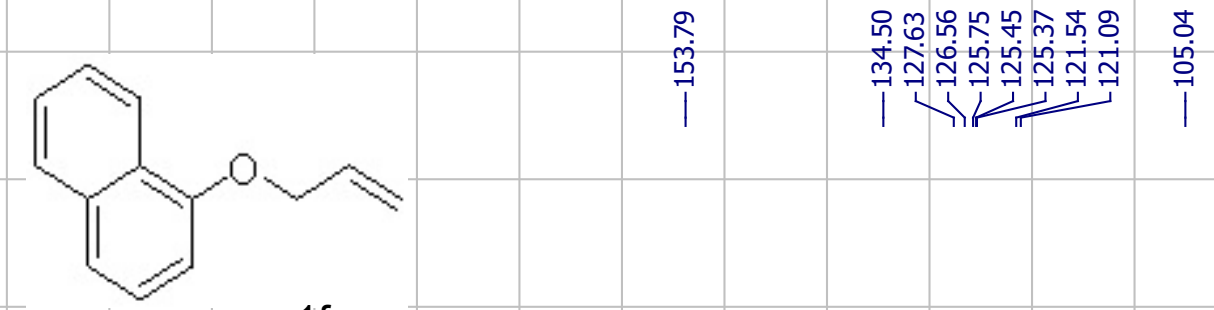

\section{ஃ.}




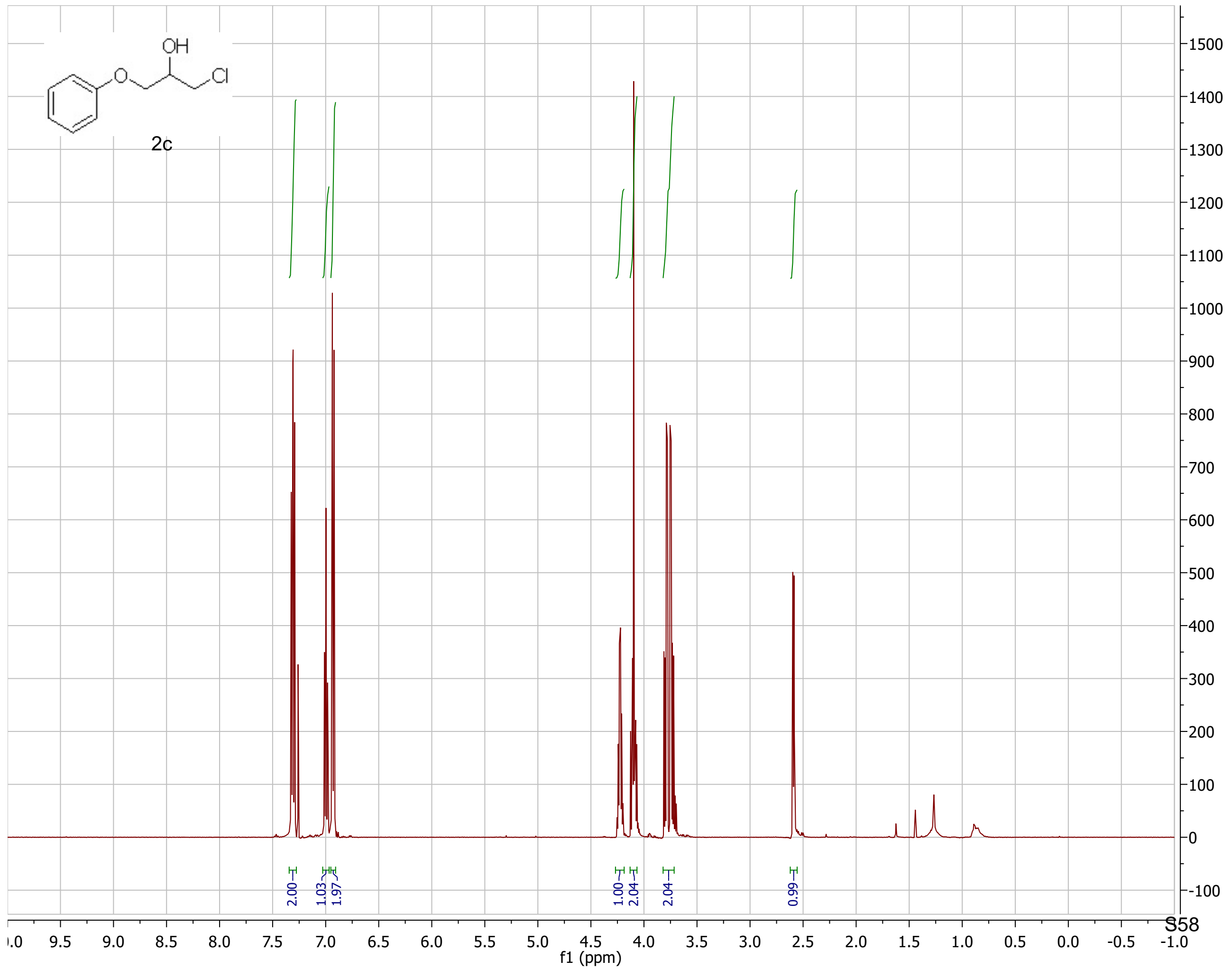




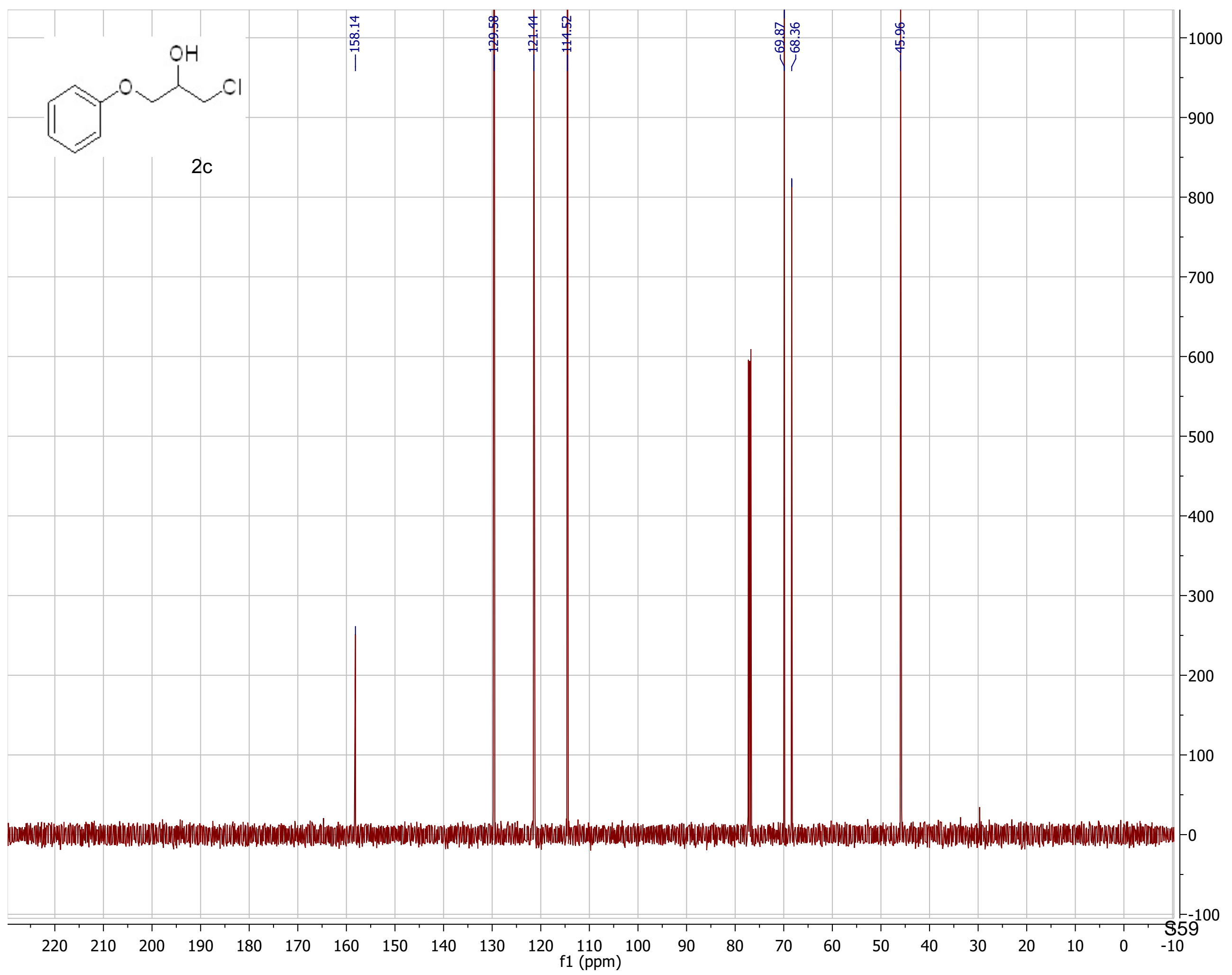




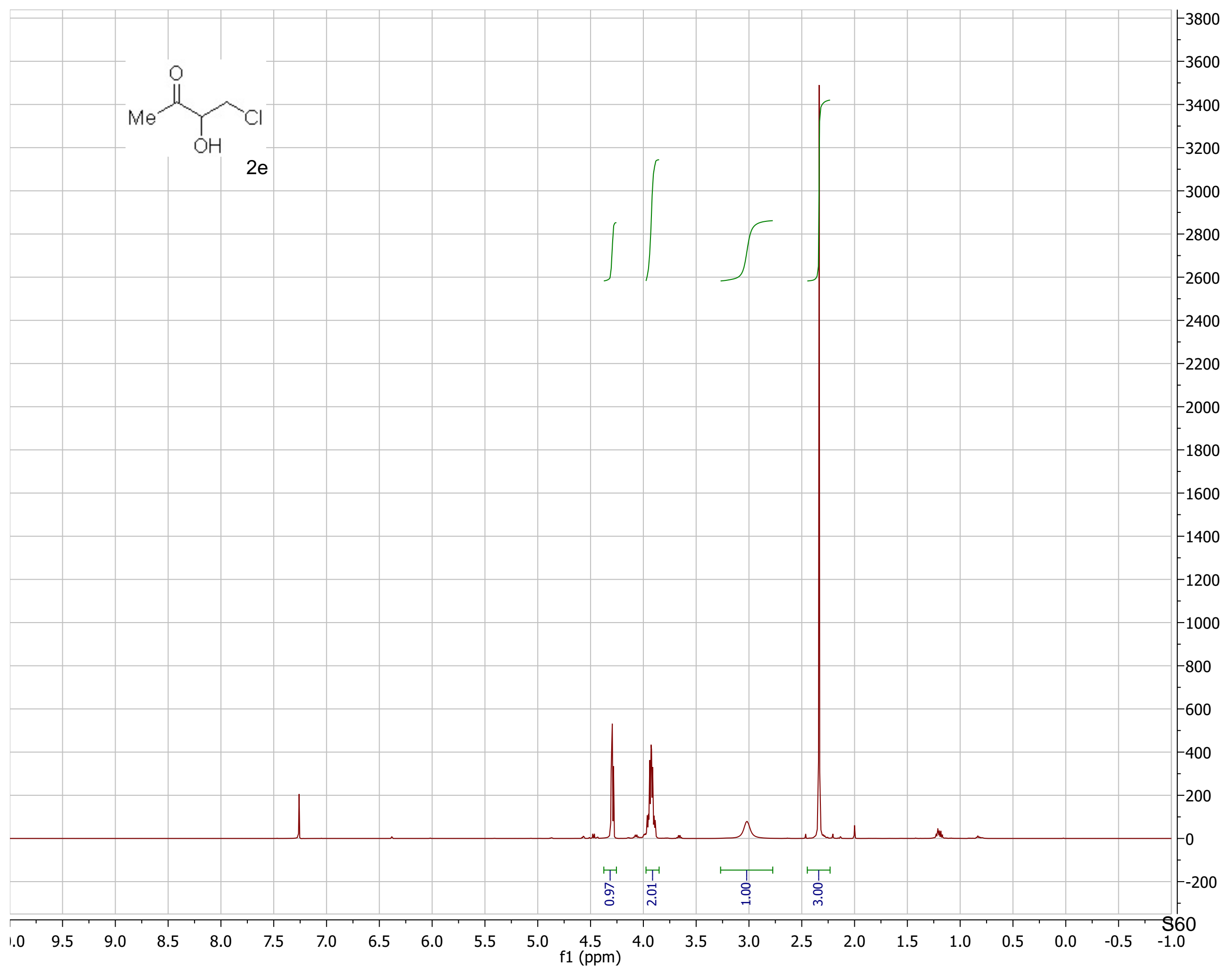




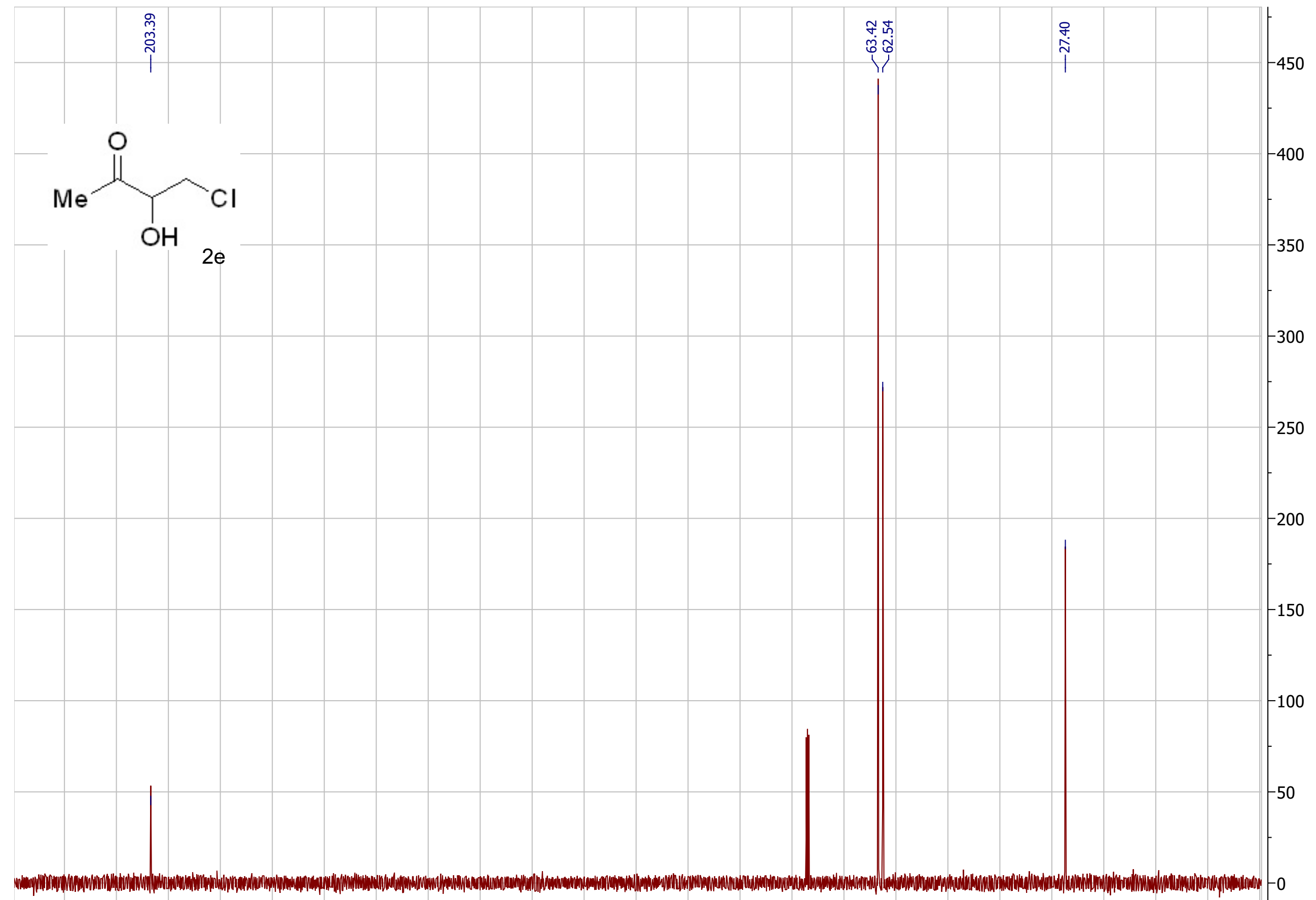

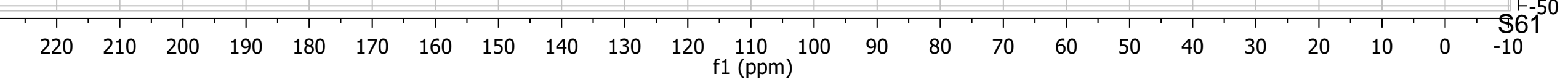




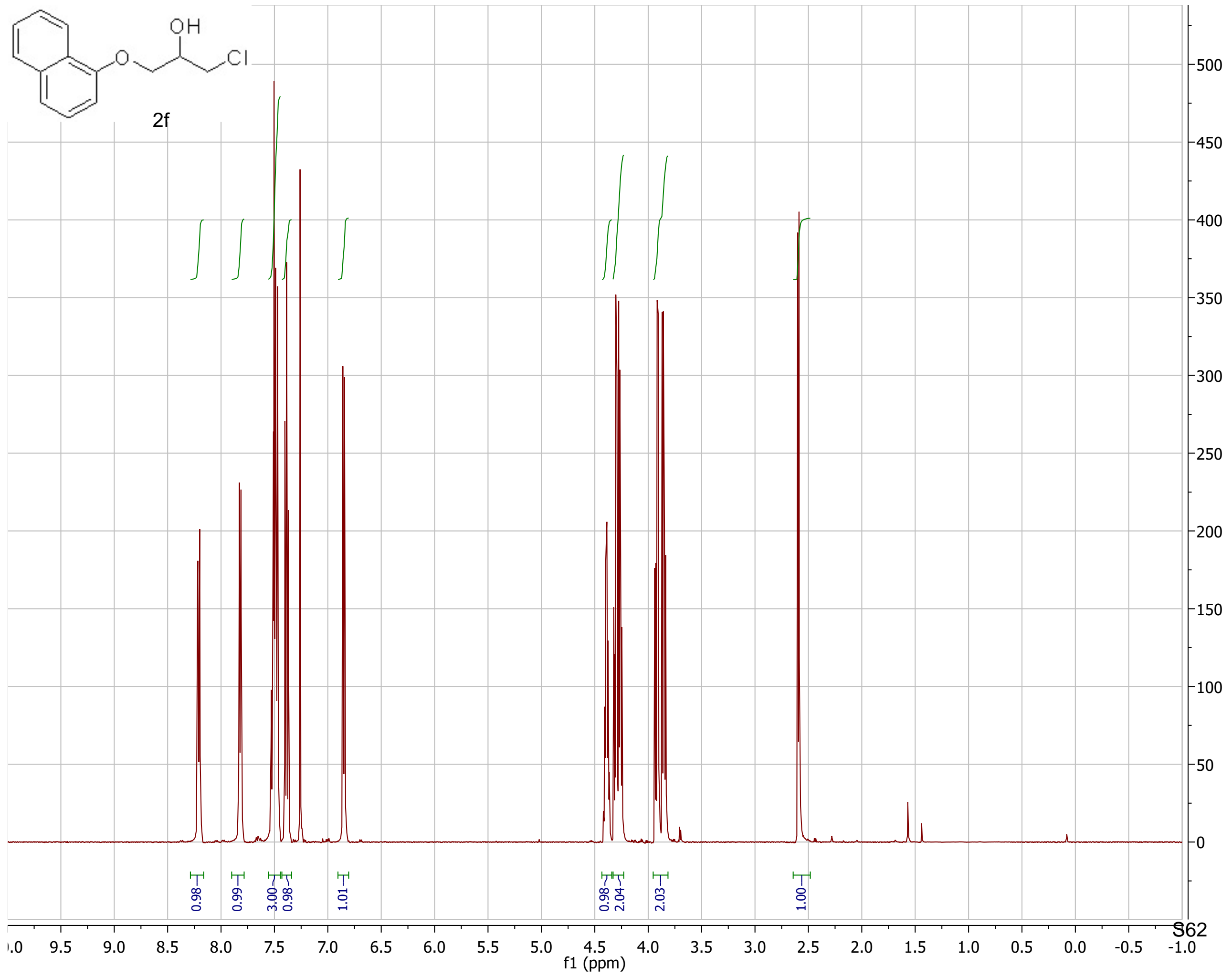




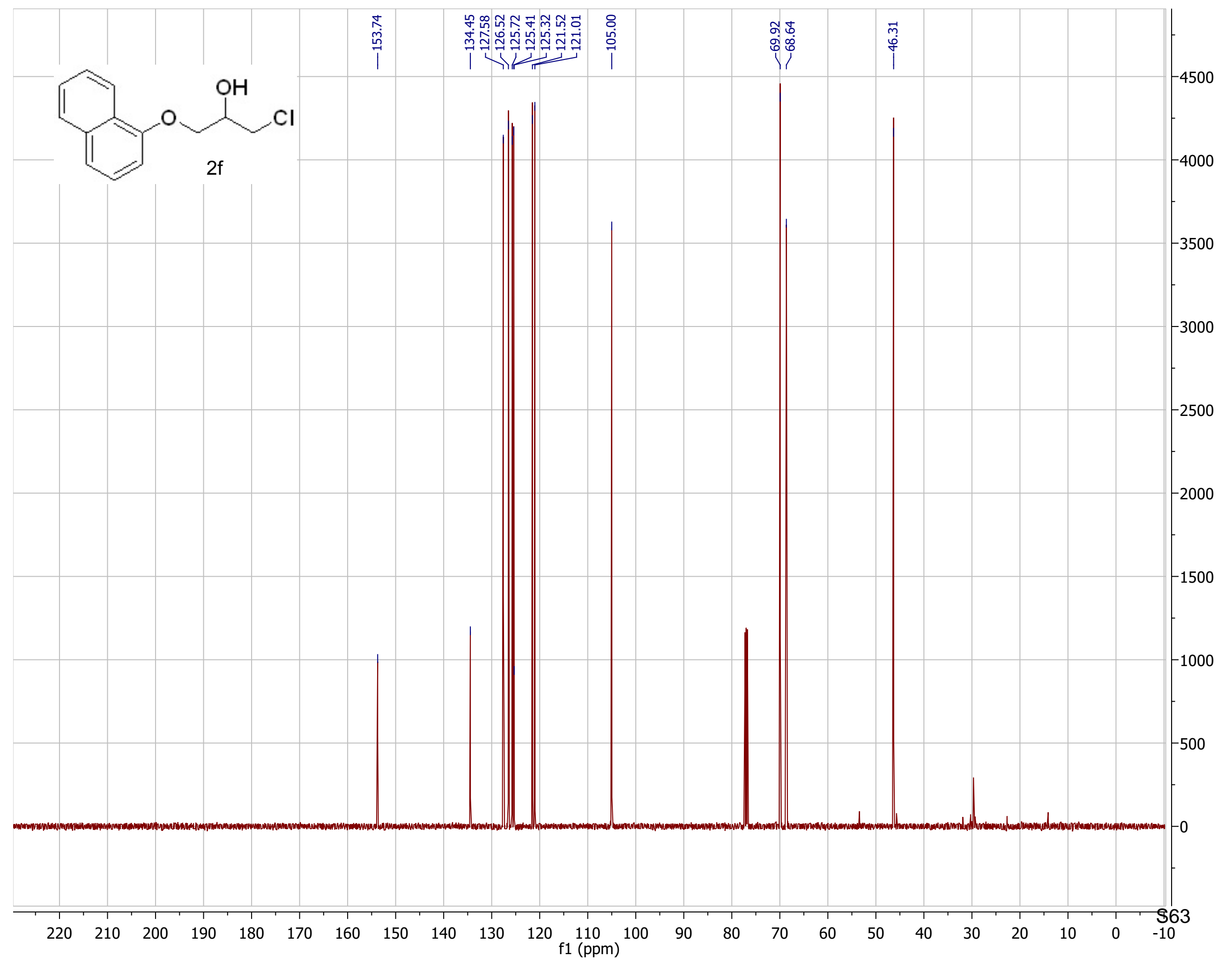




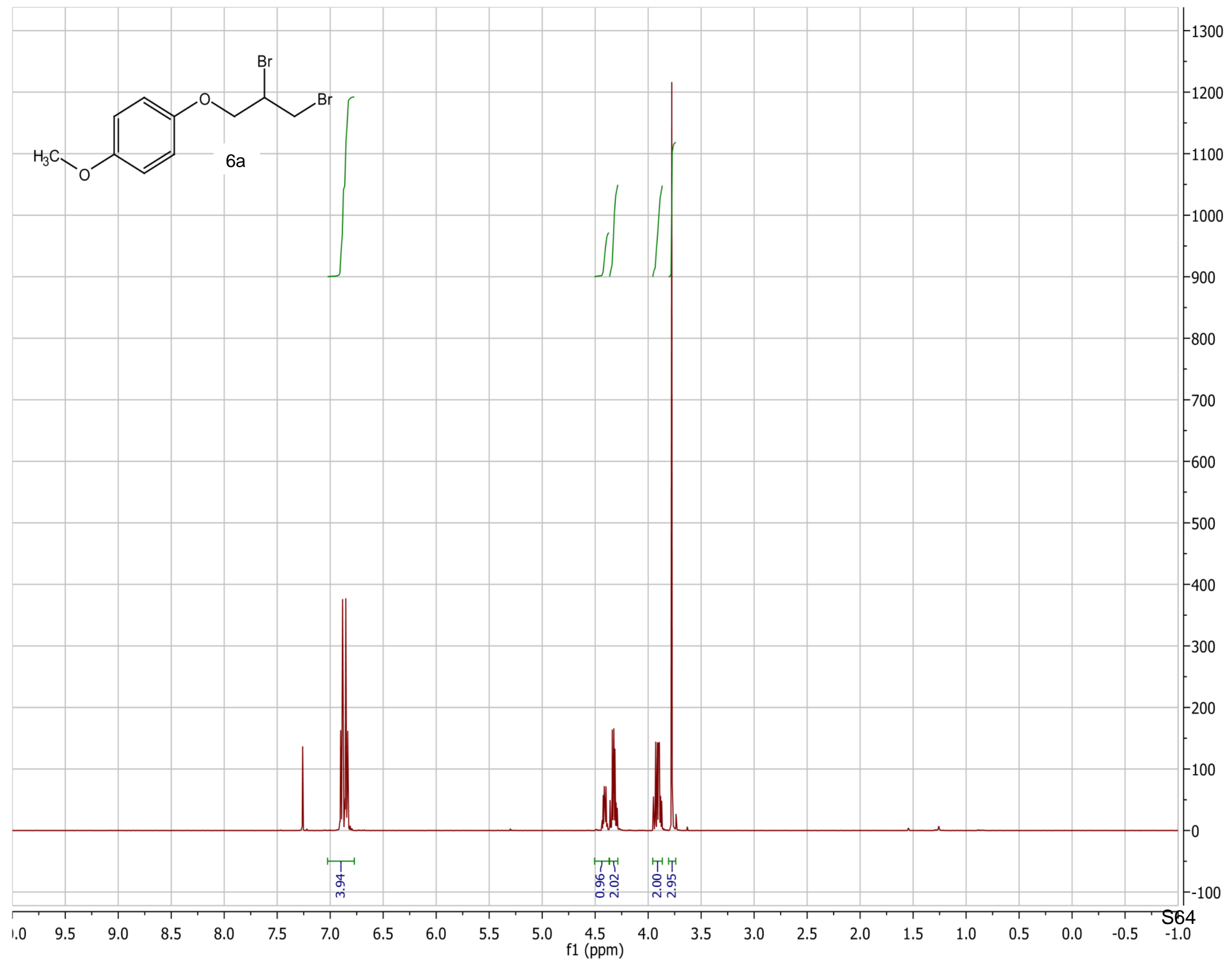




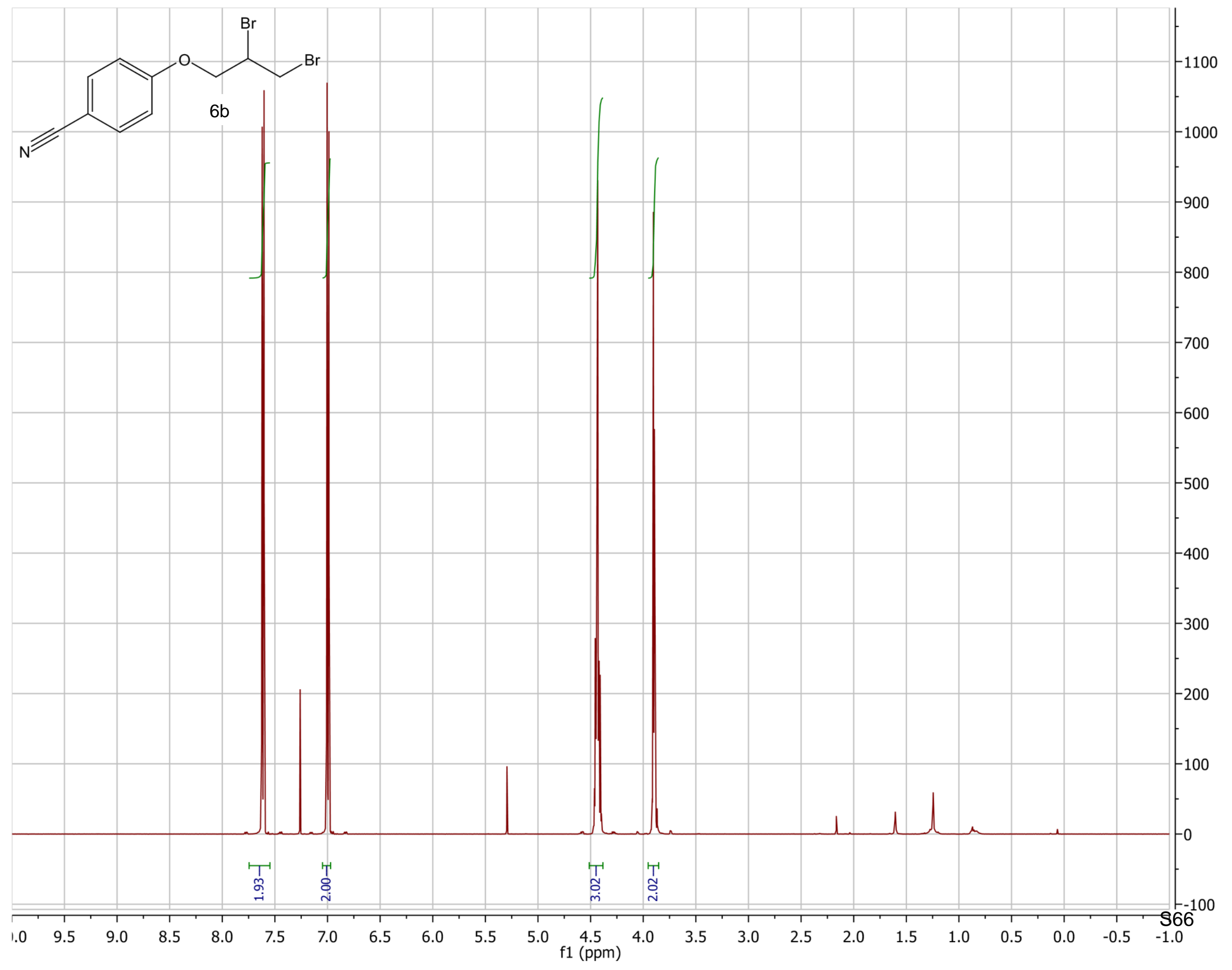



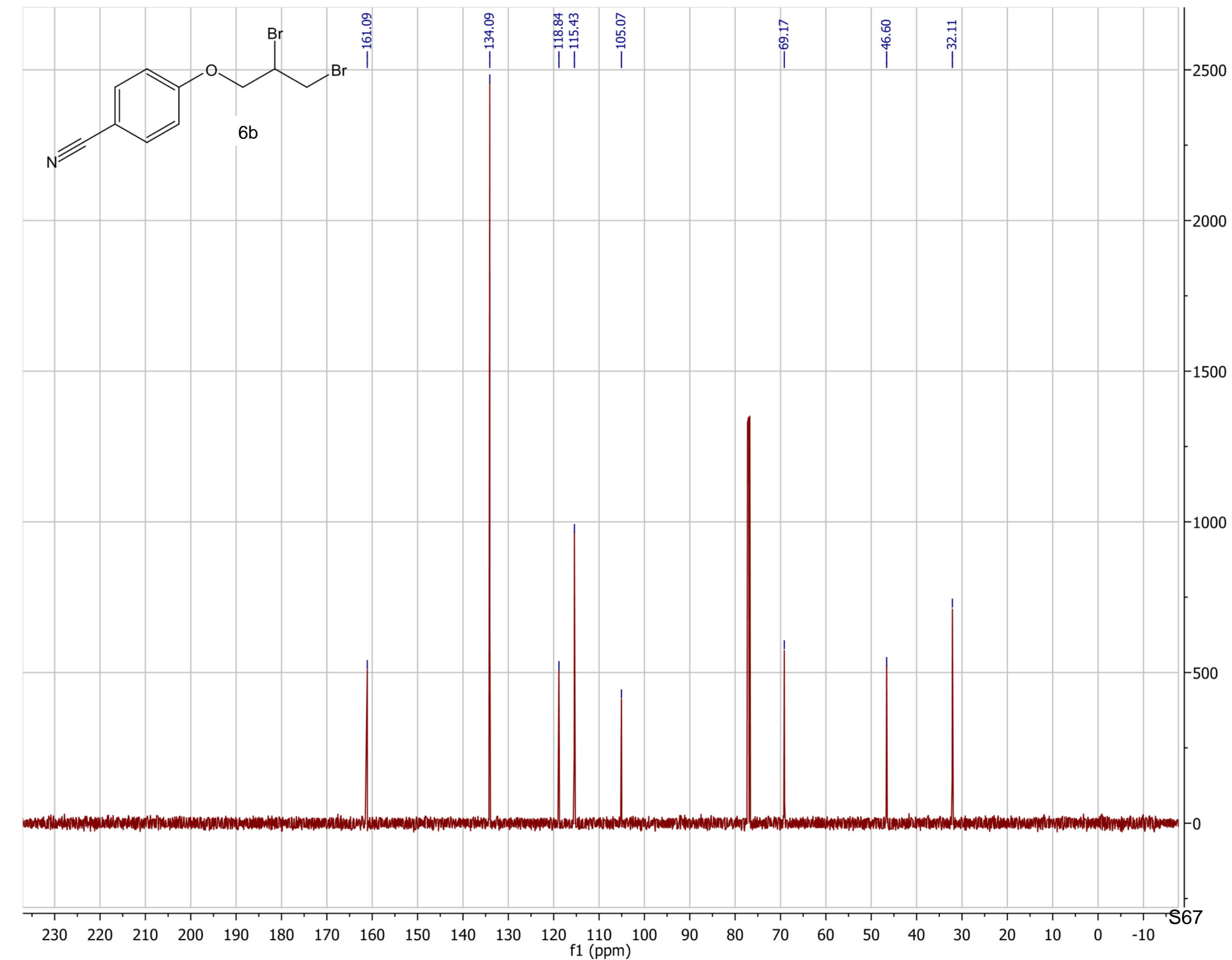


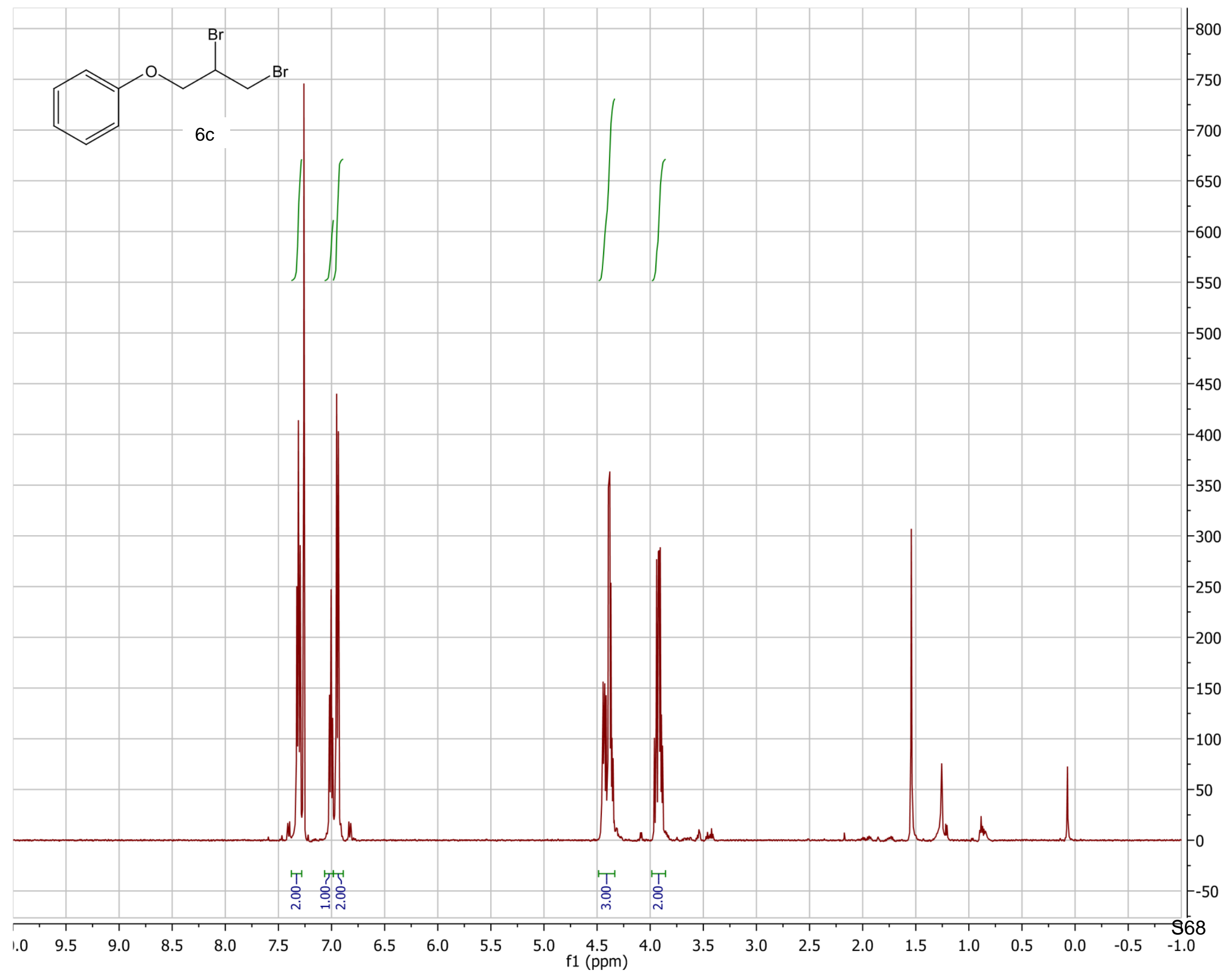



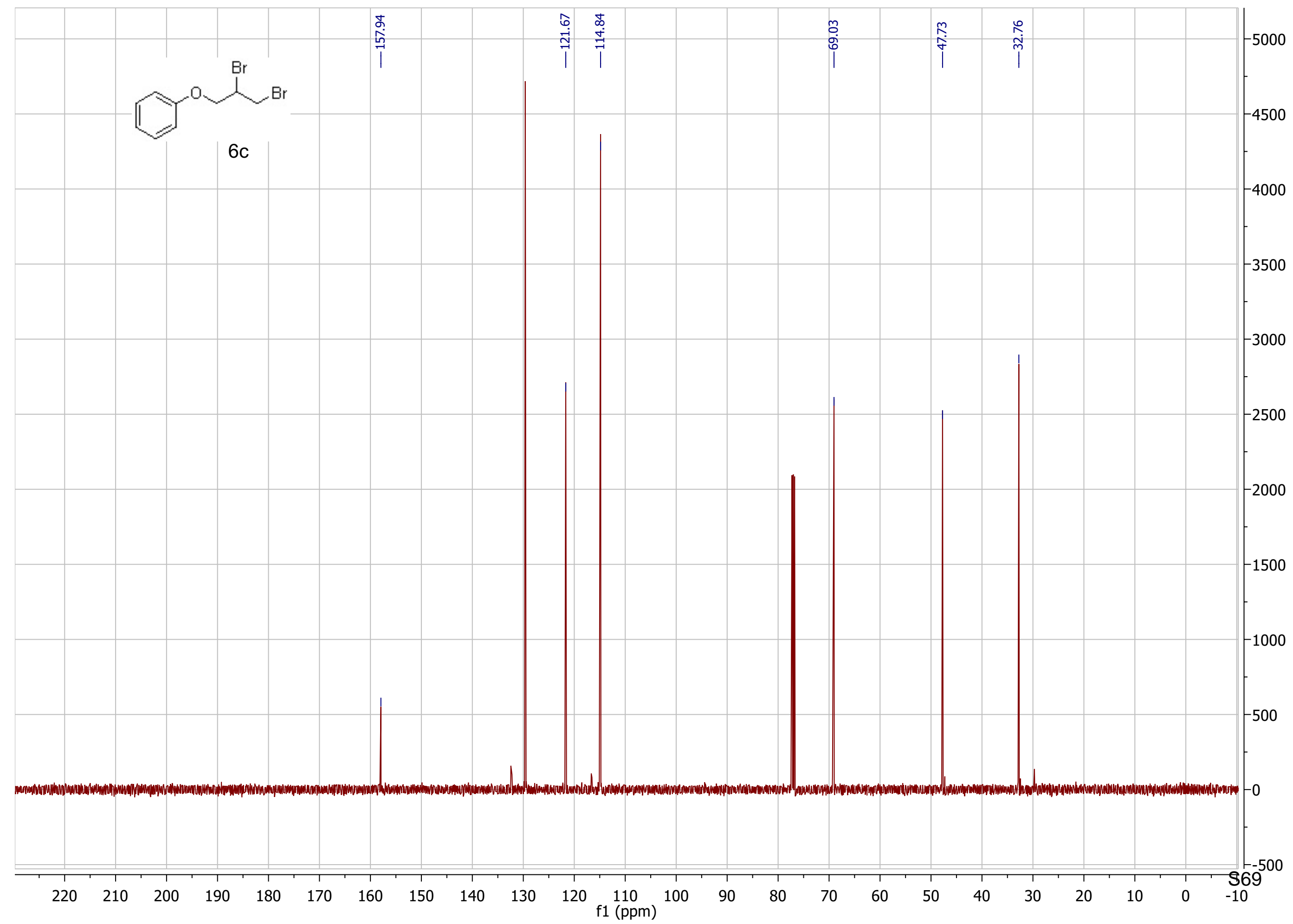


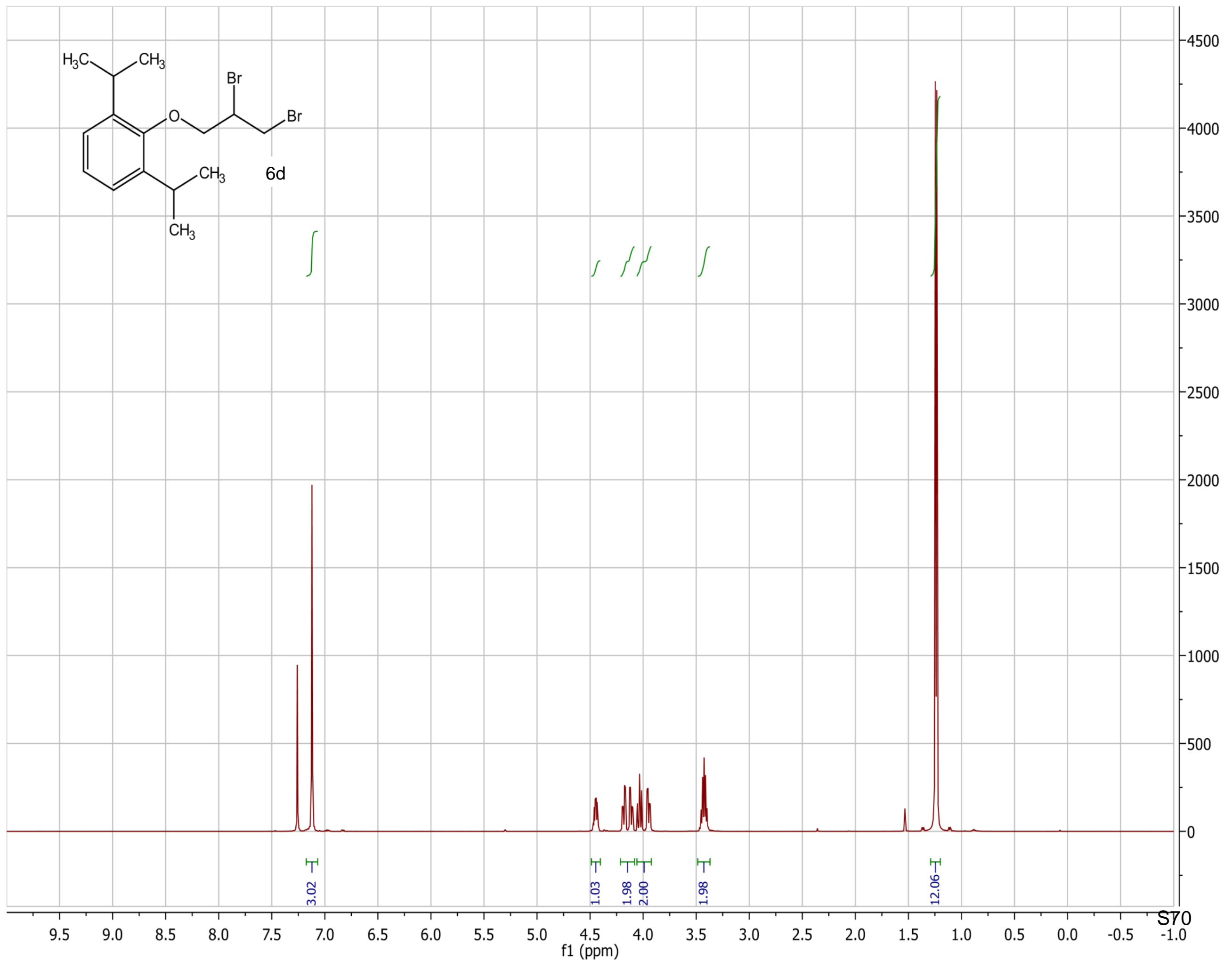




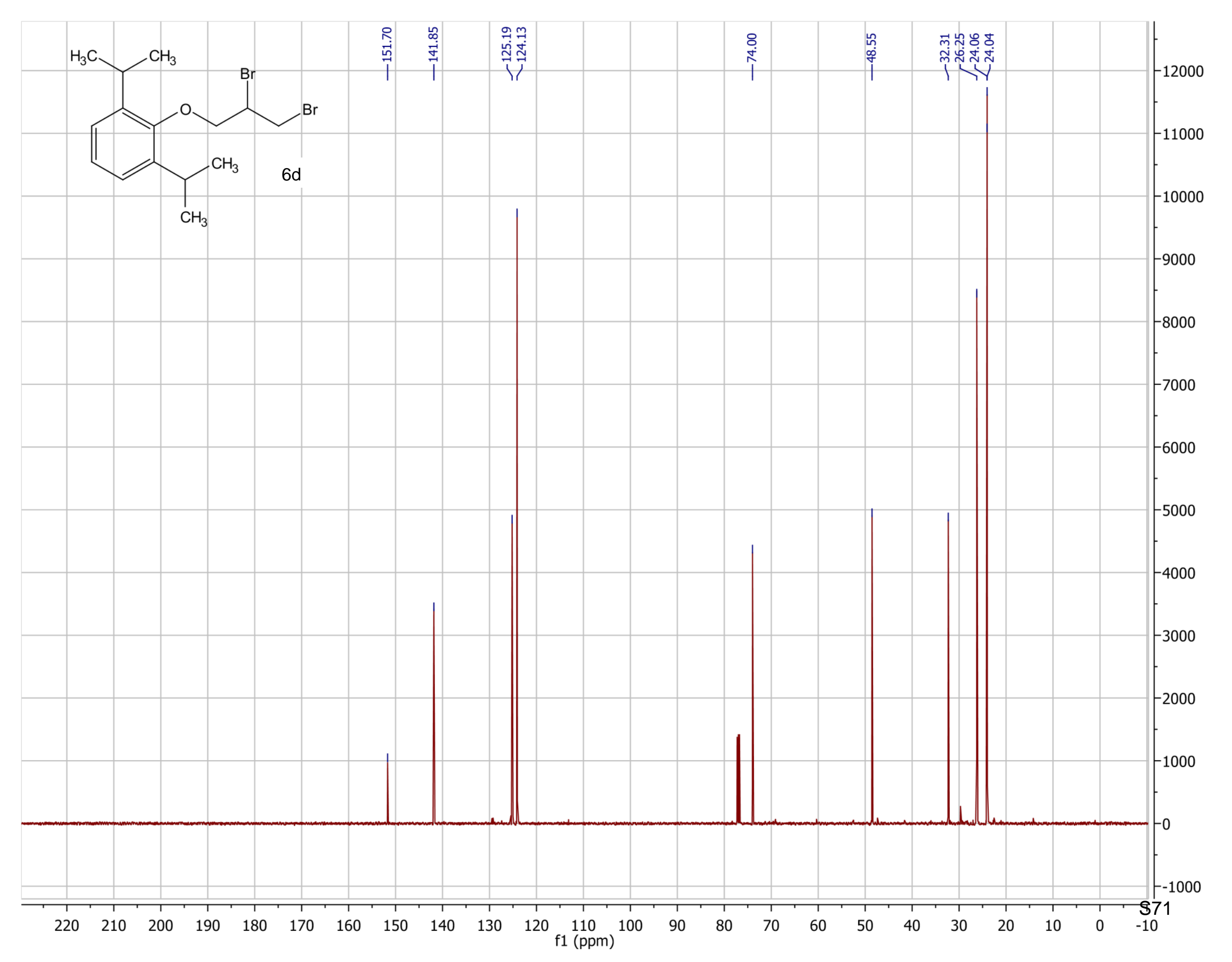




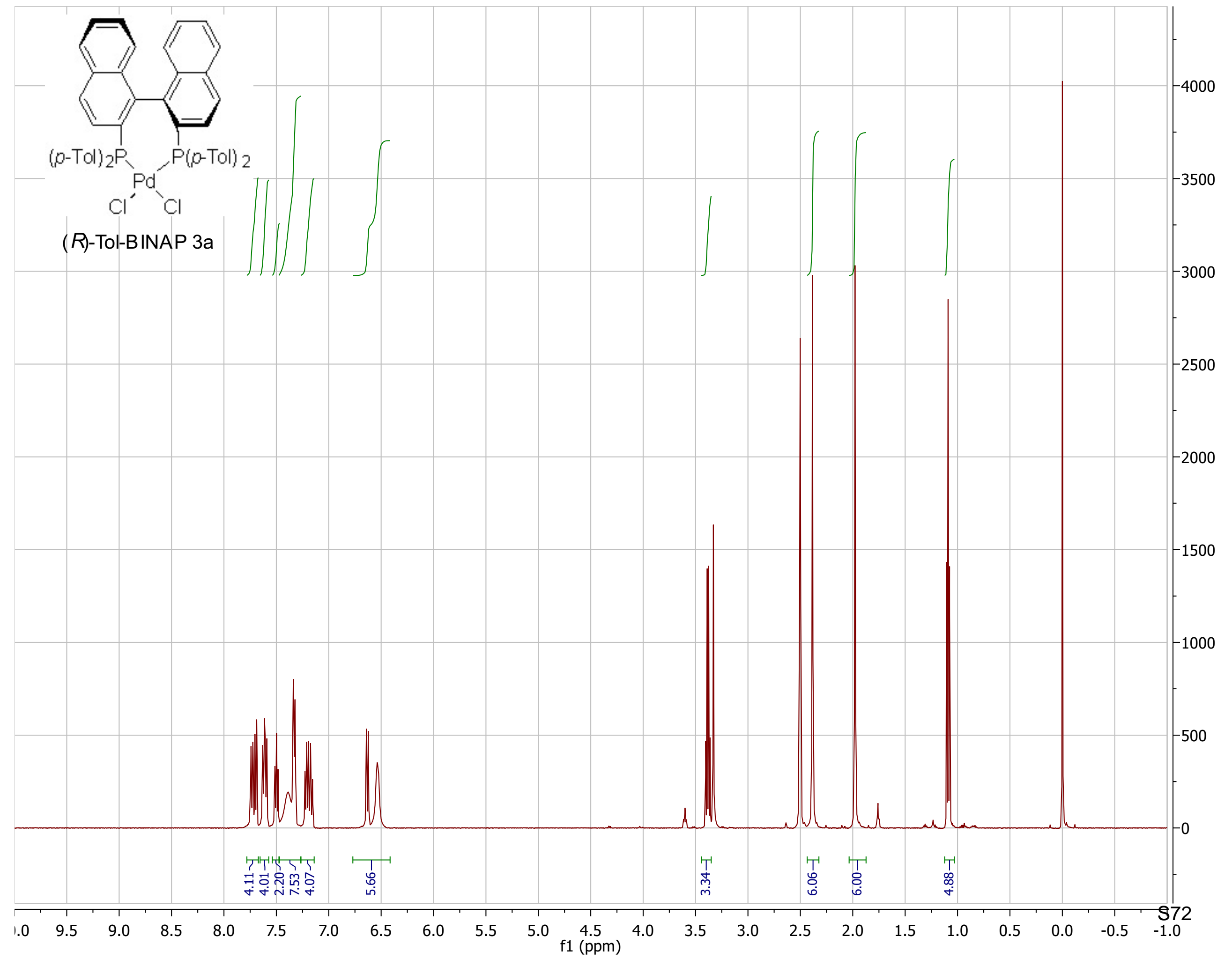




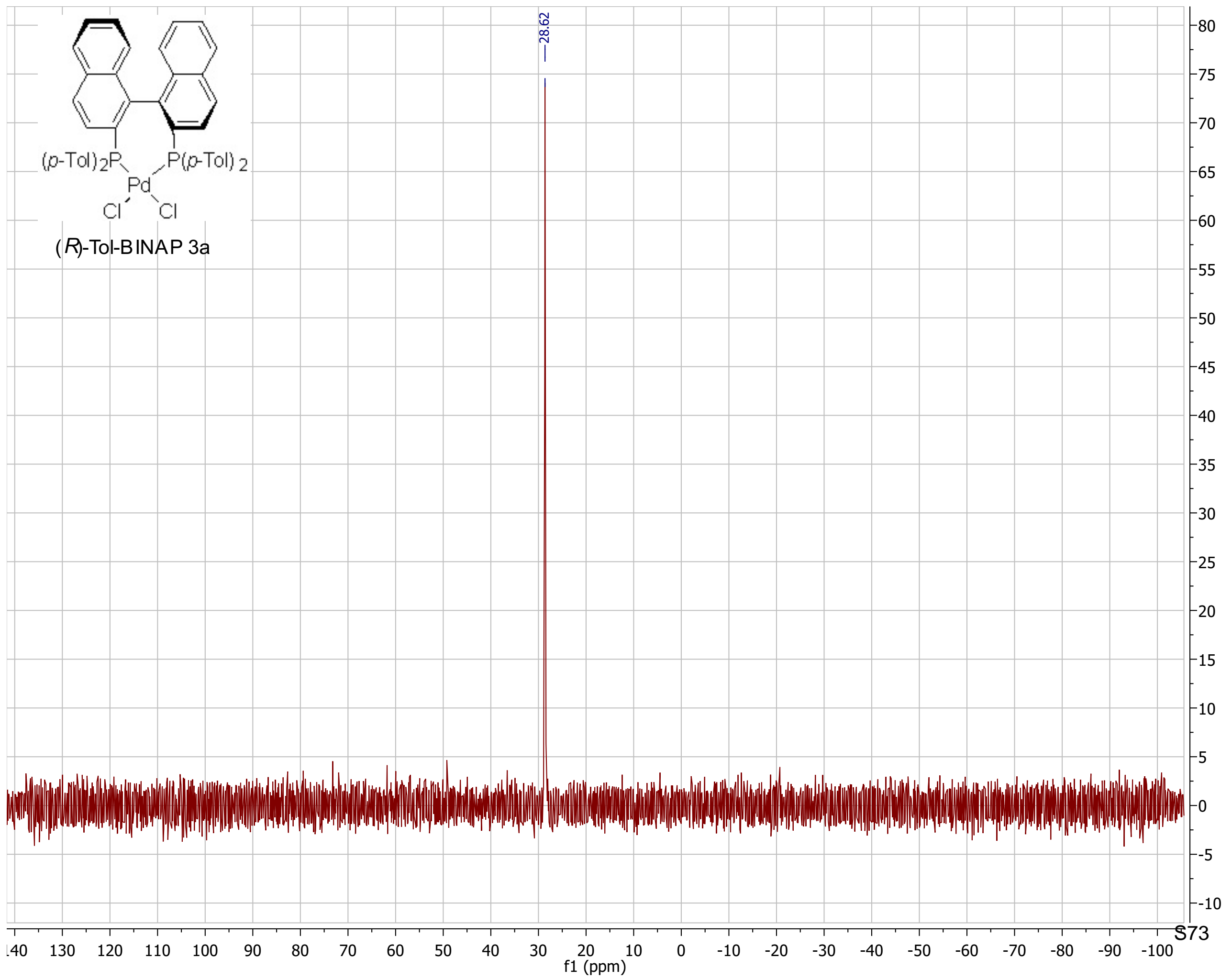




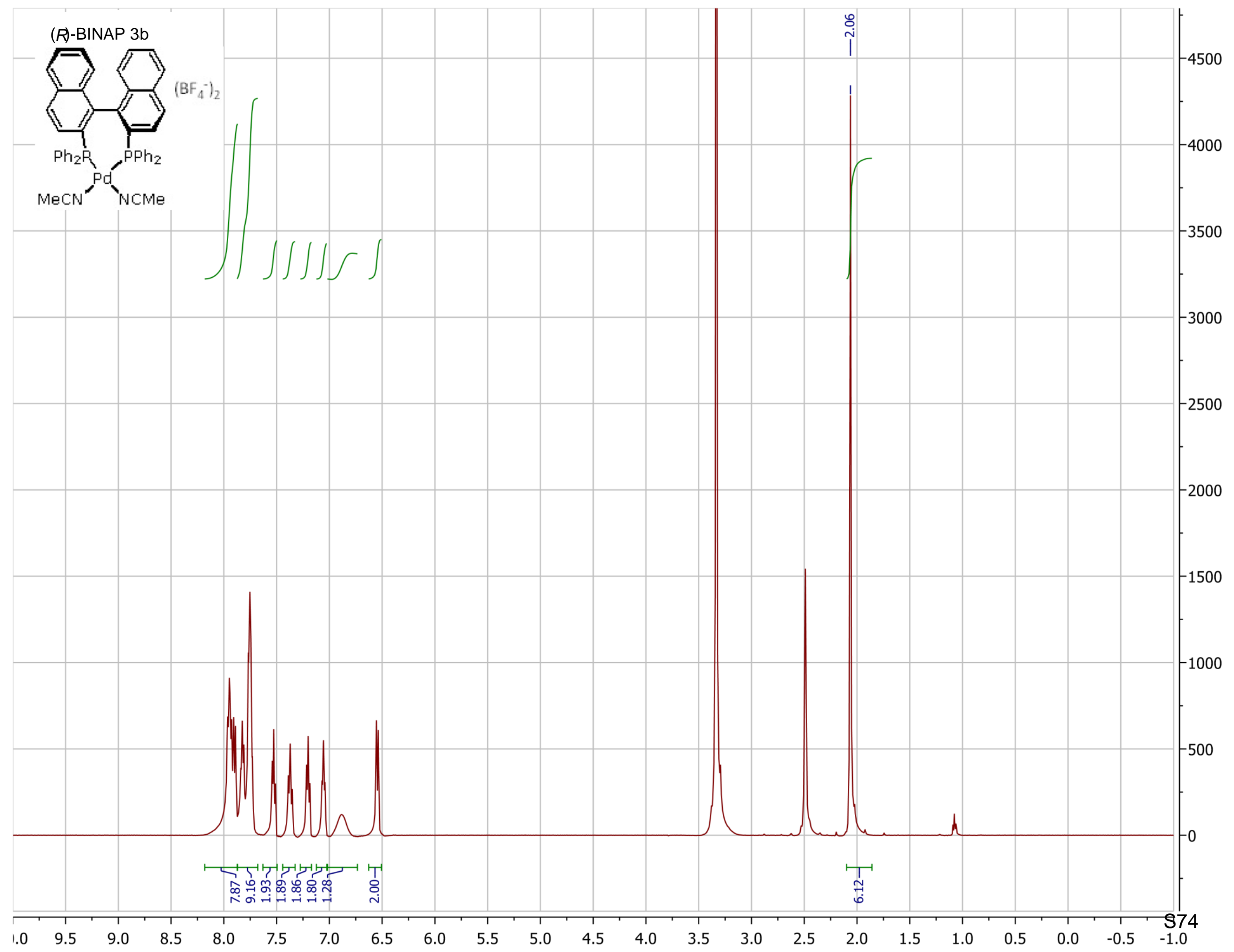




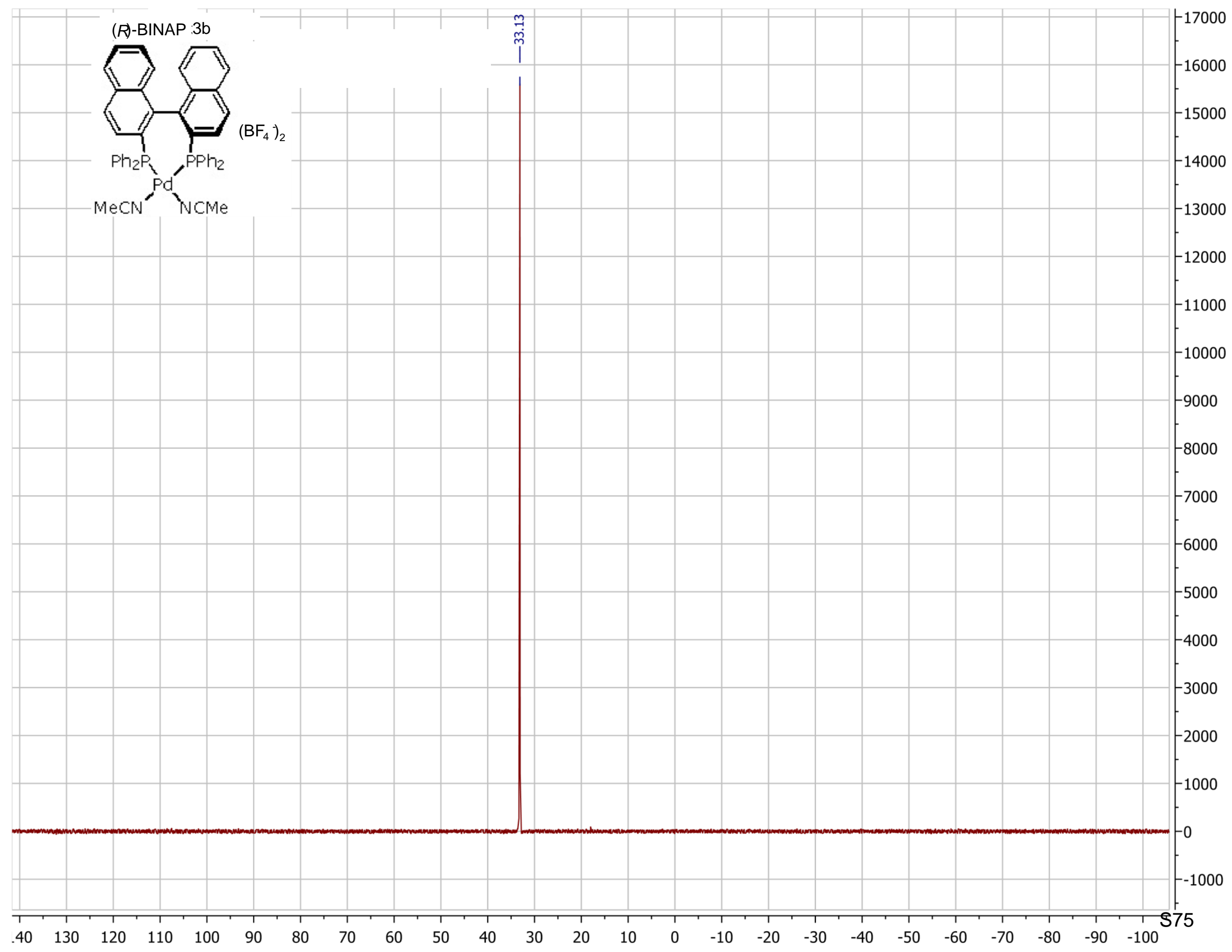




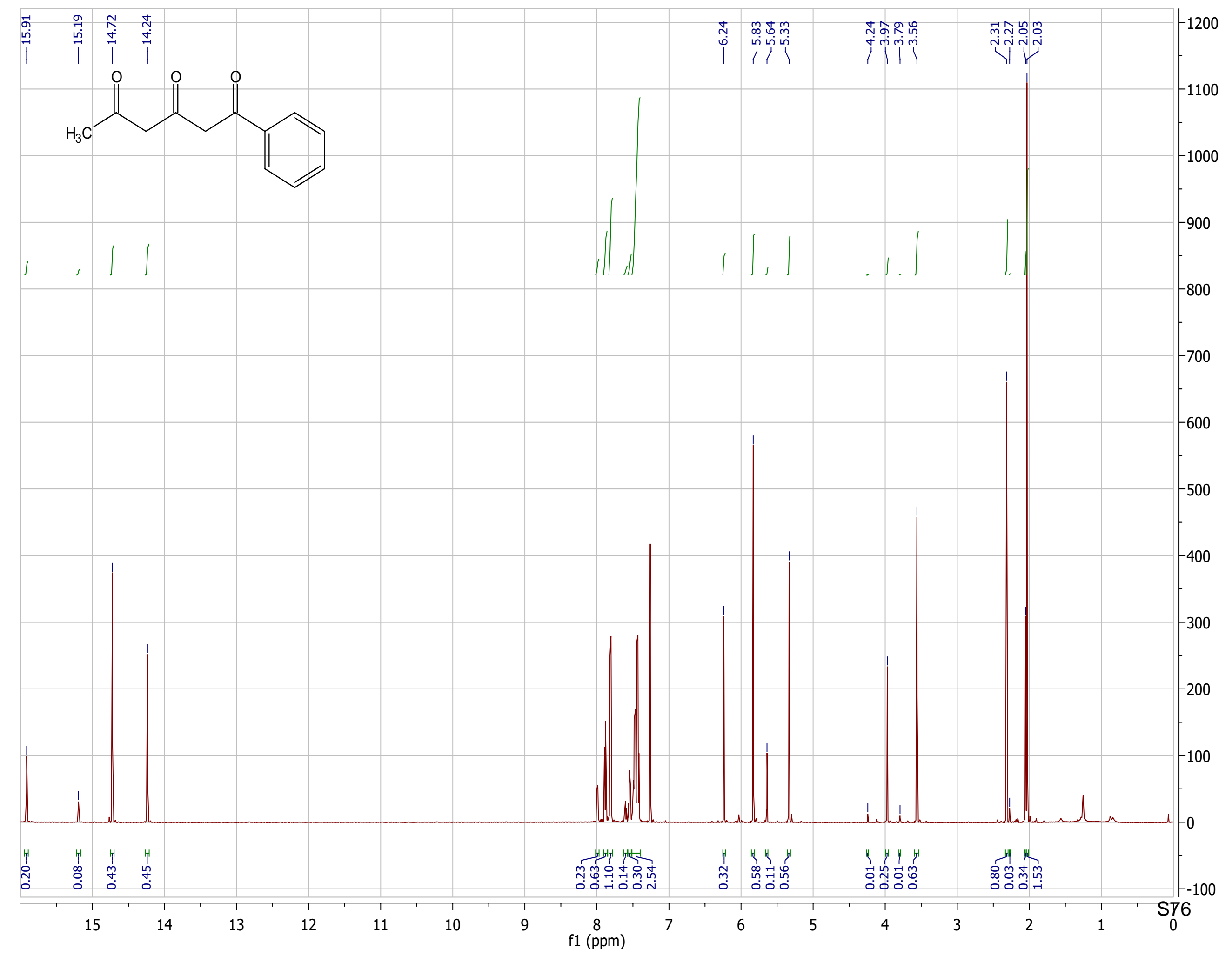




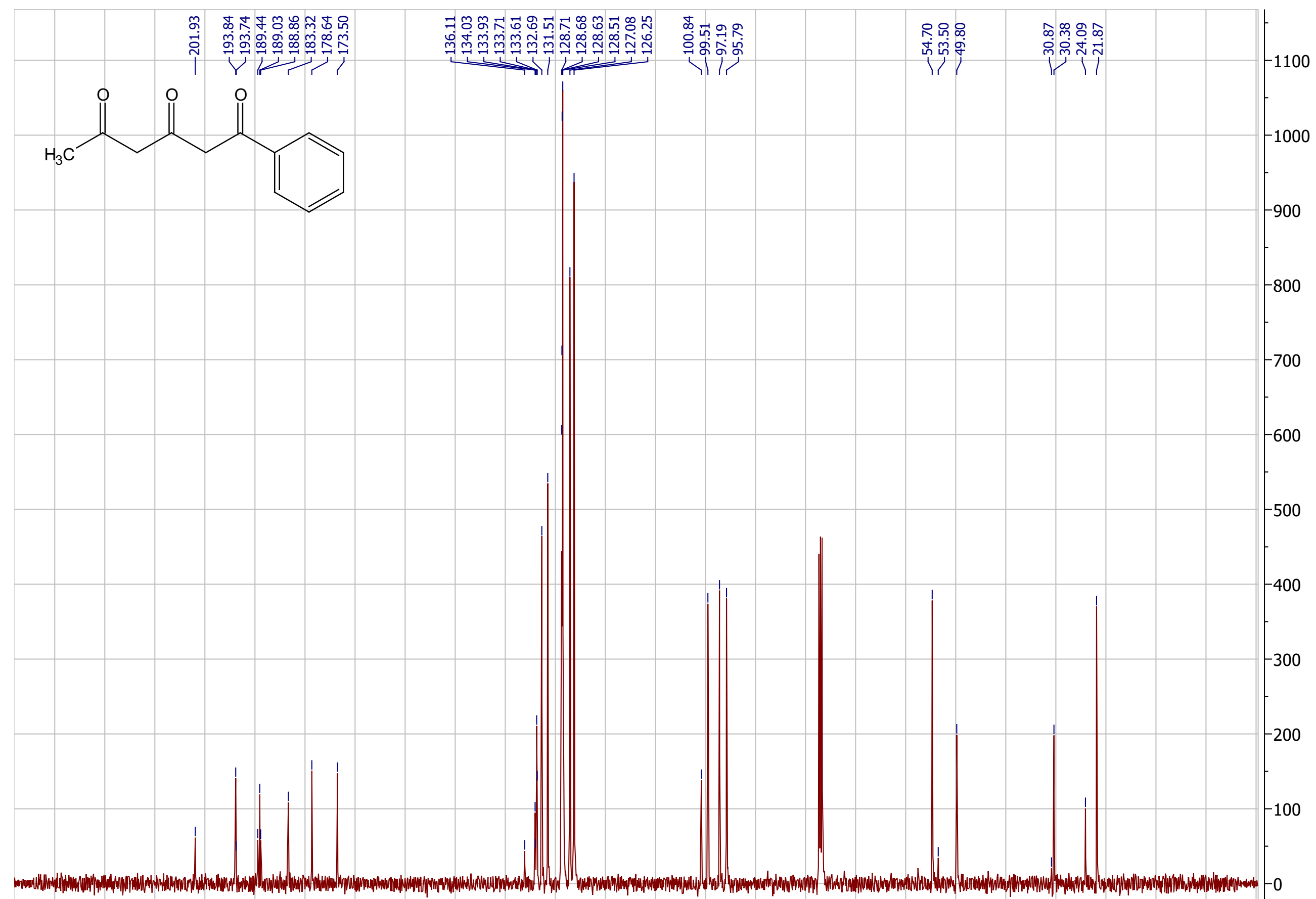




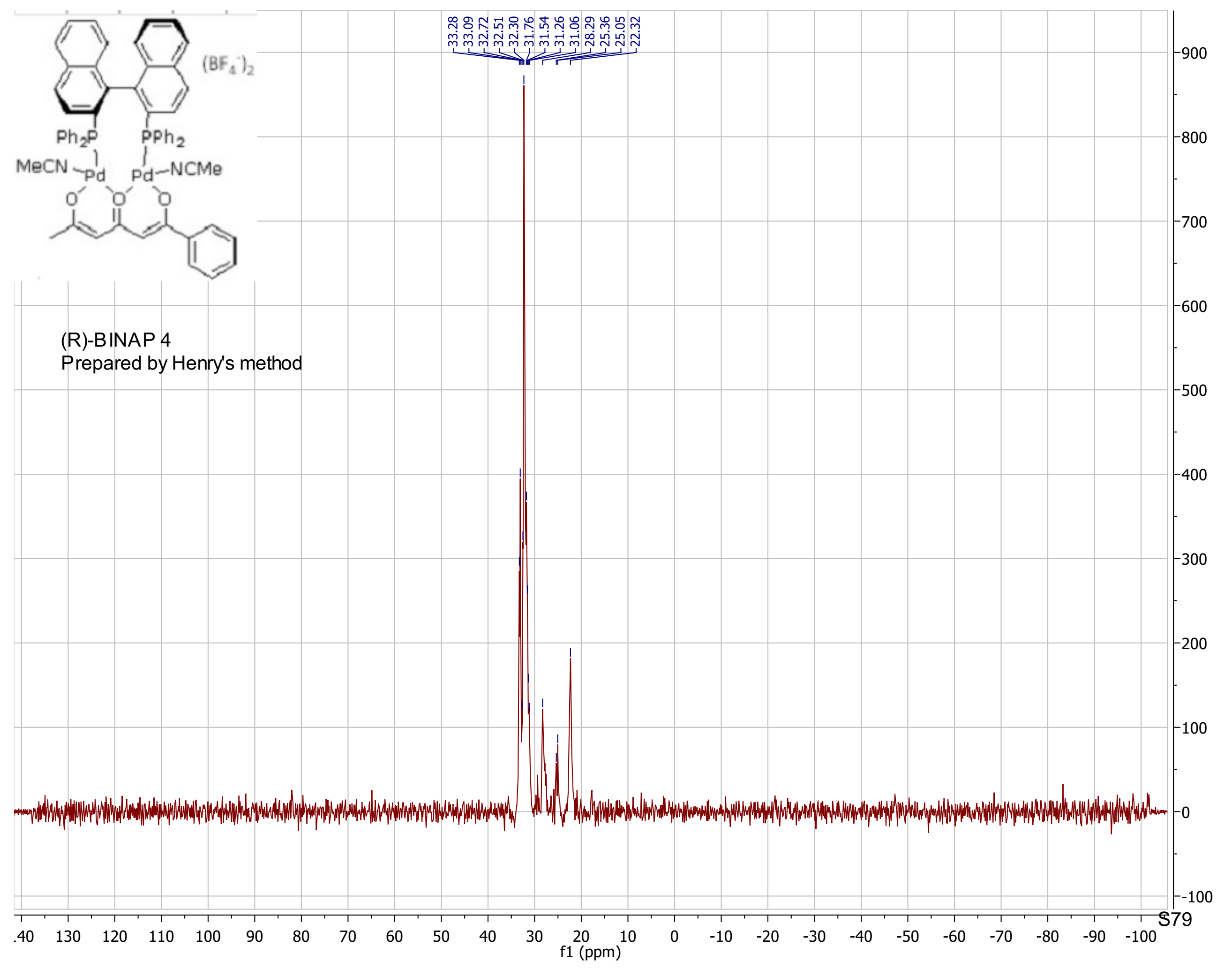




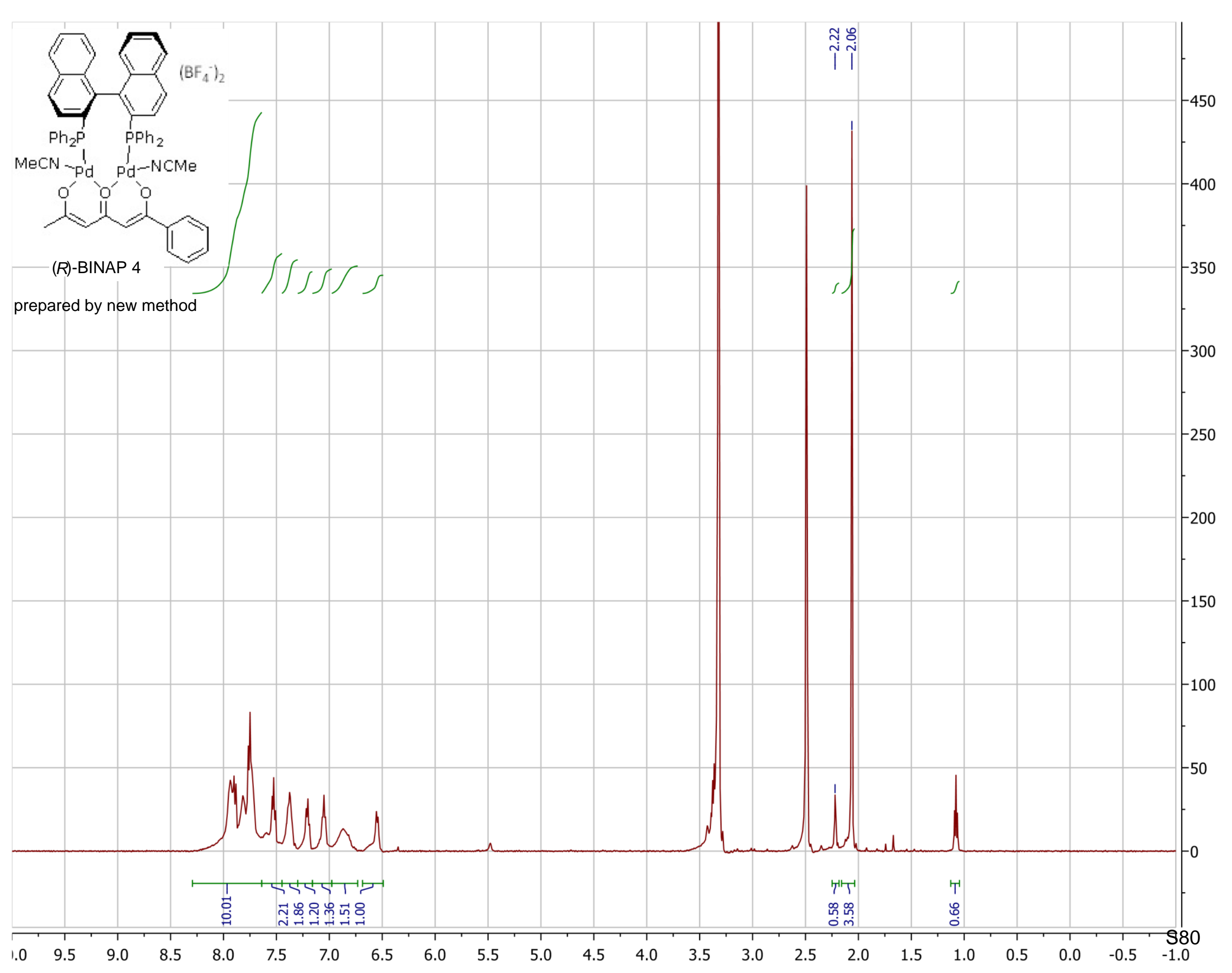




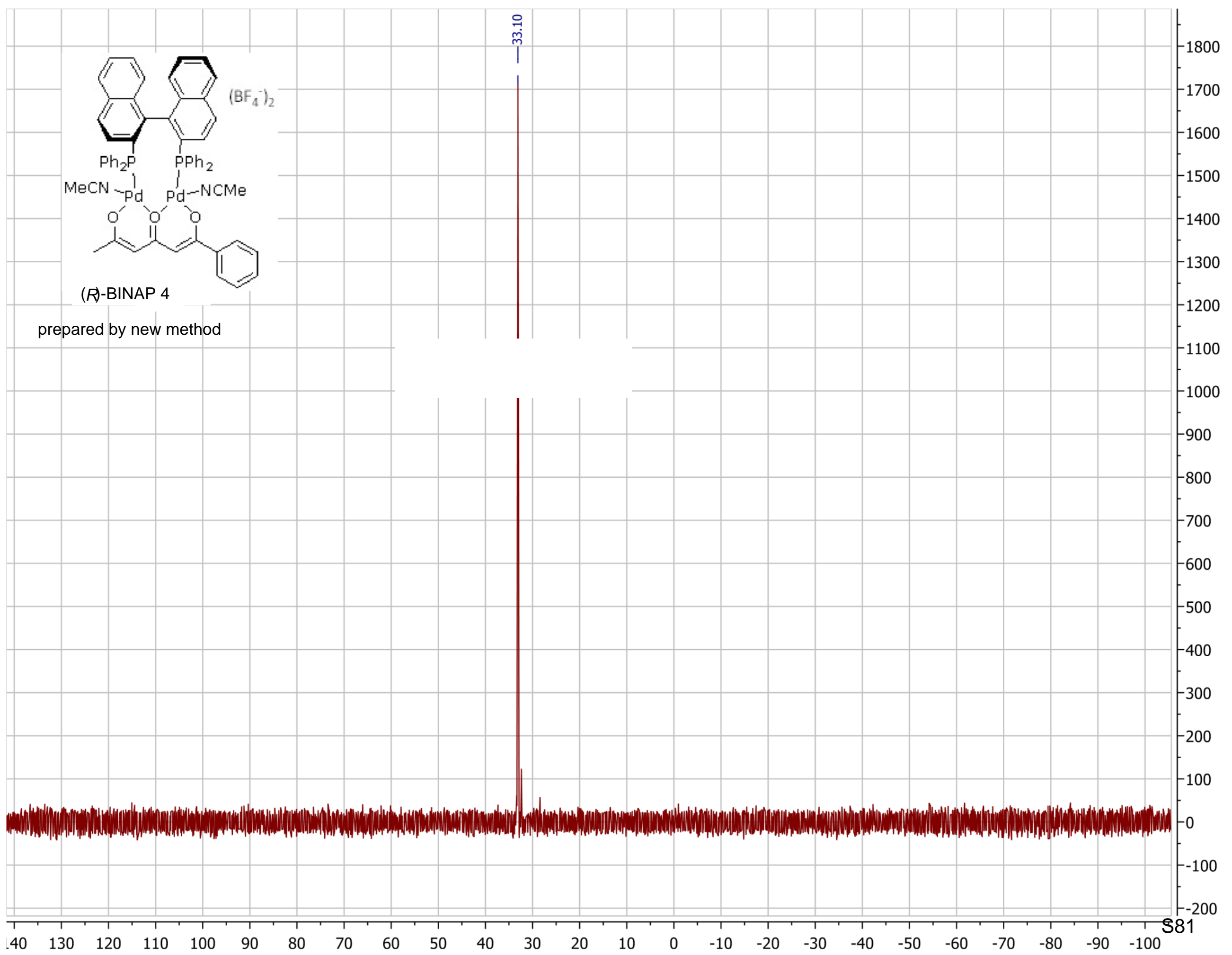




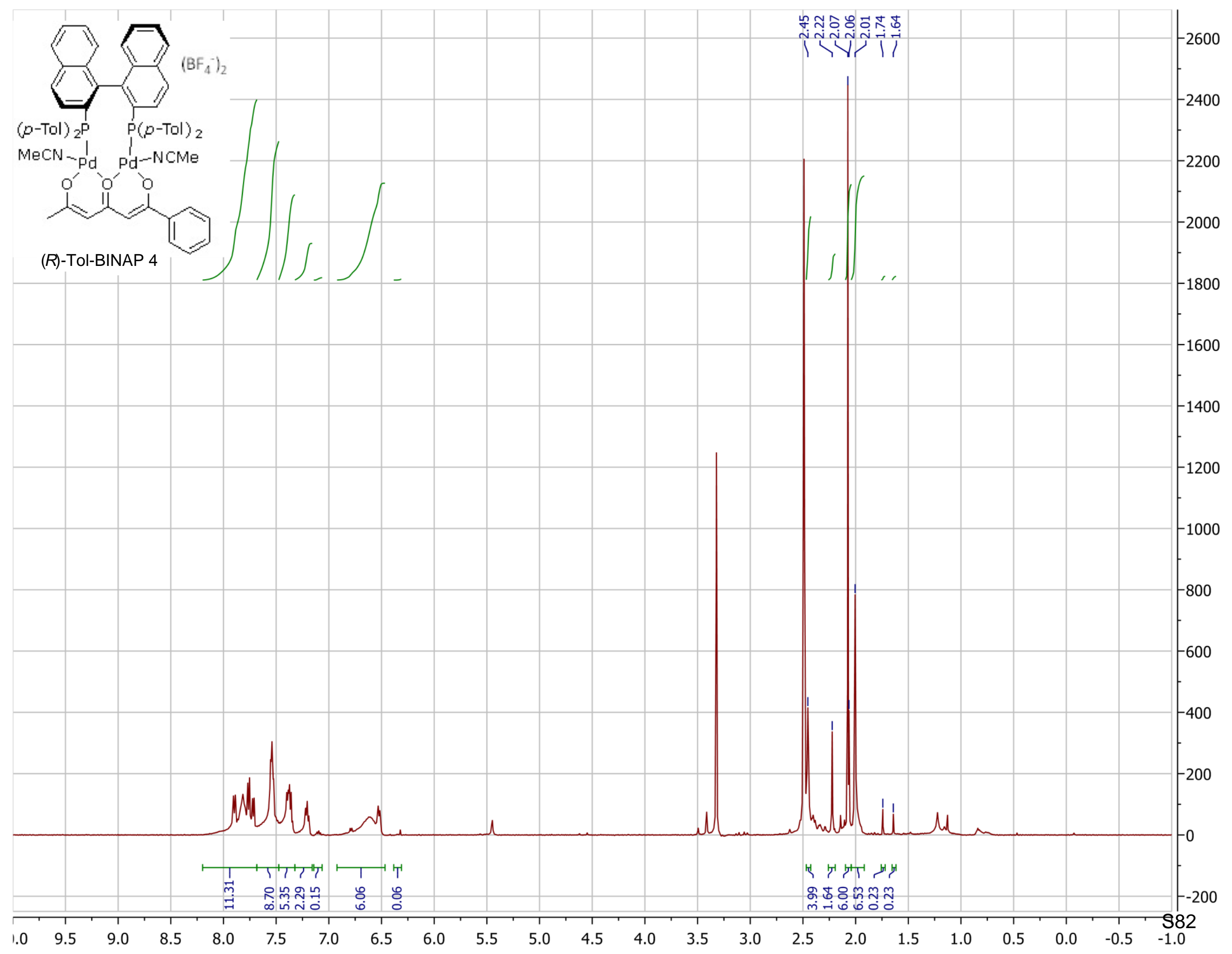




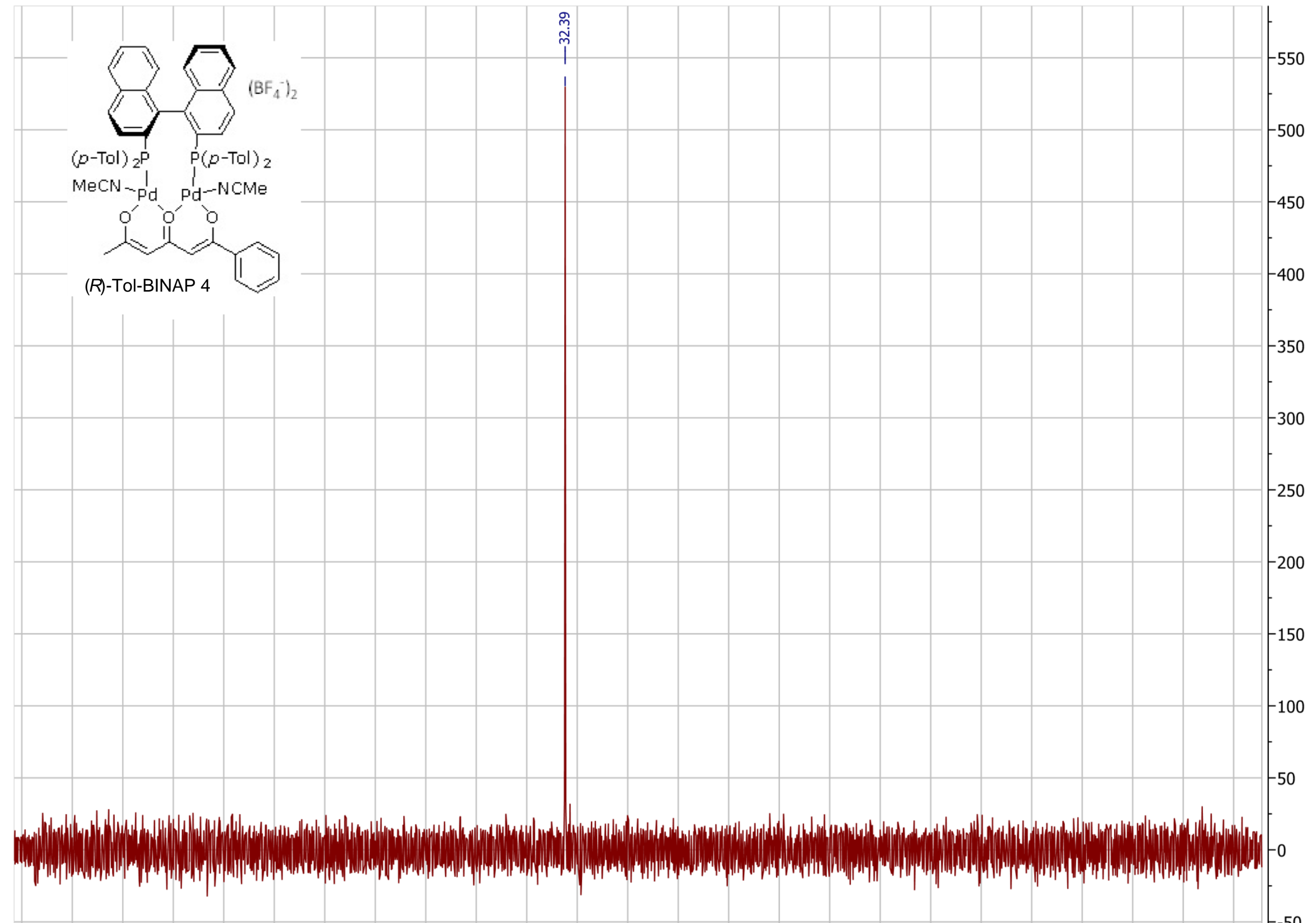

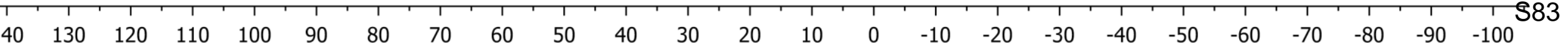




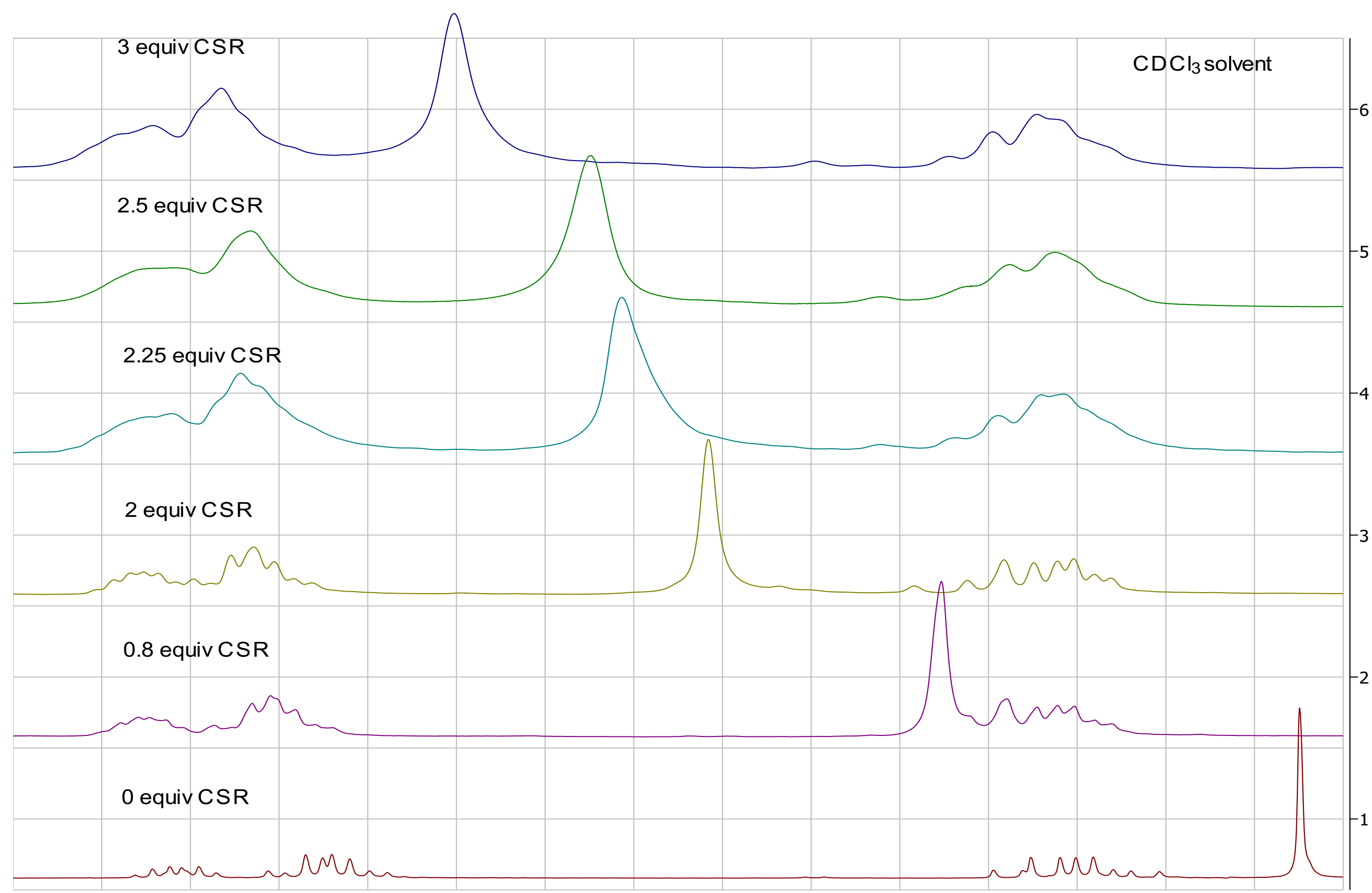

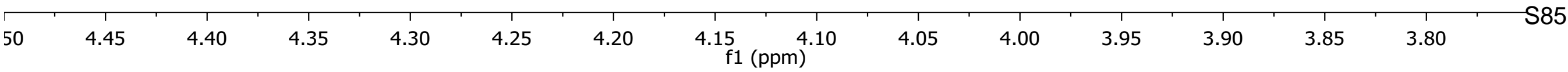




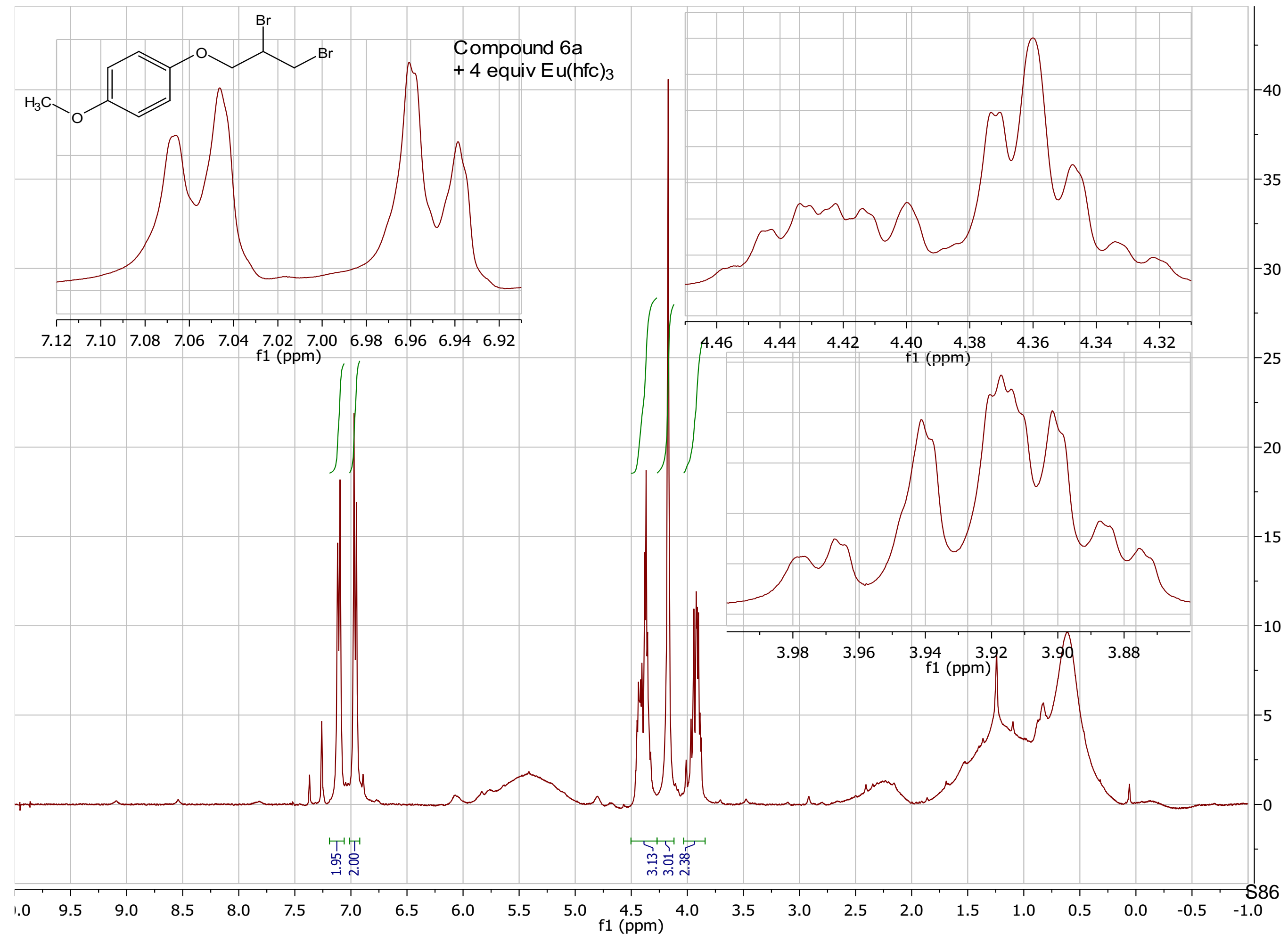




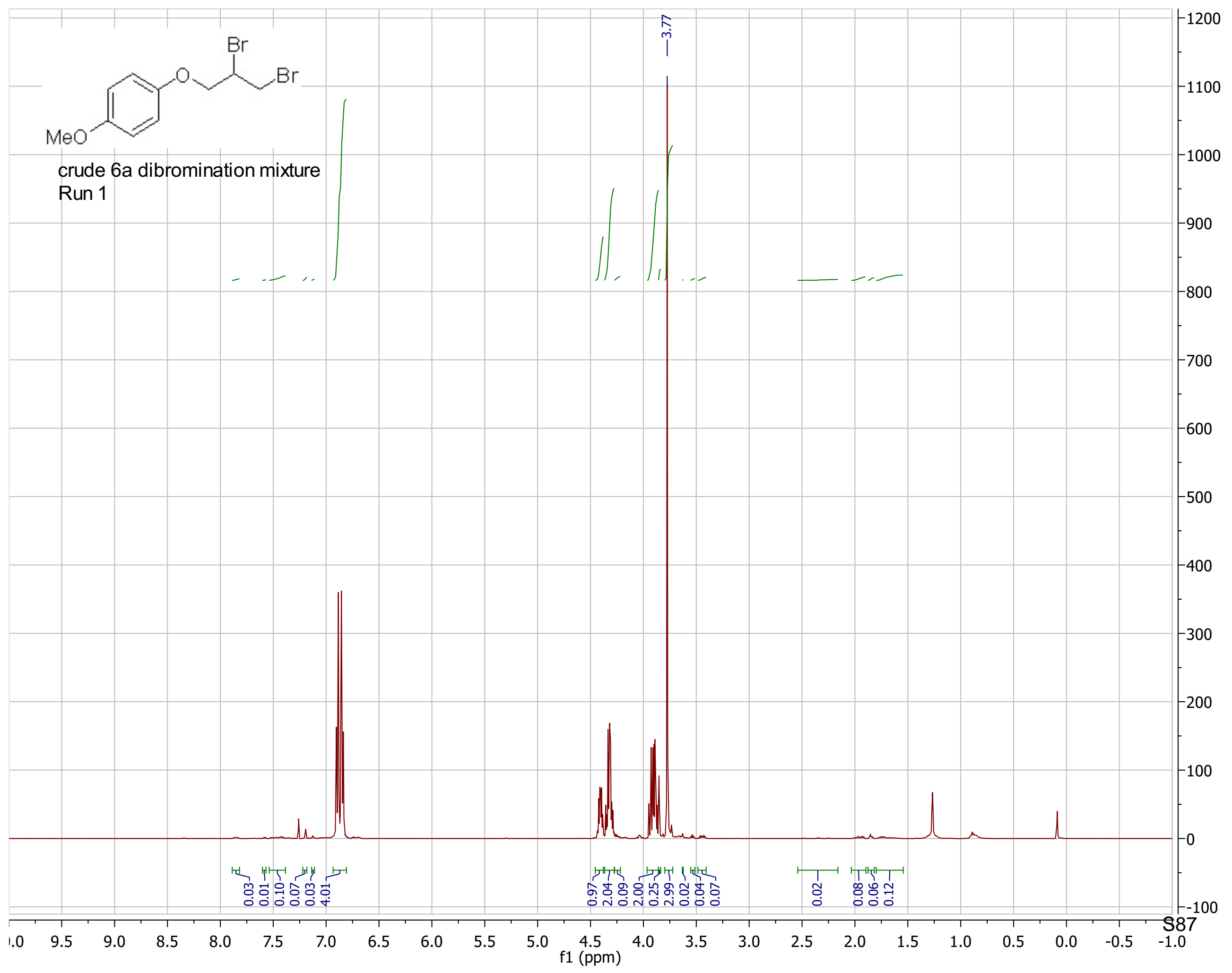




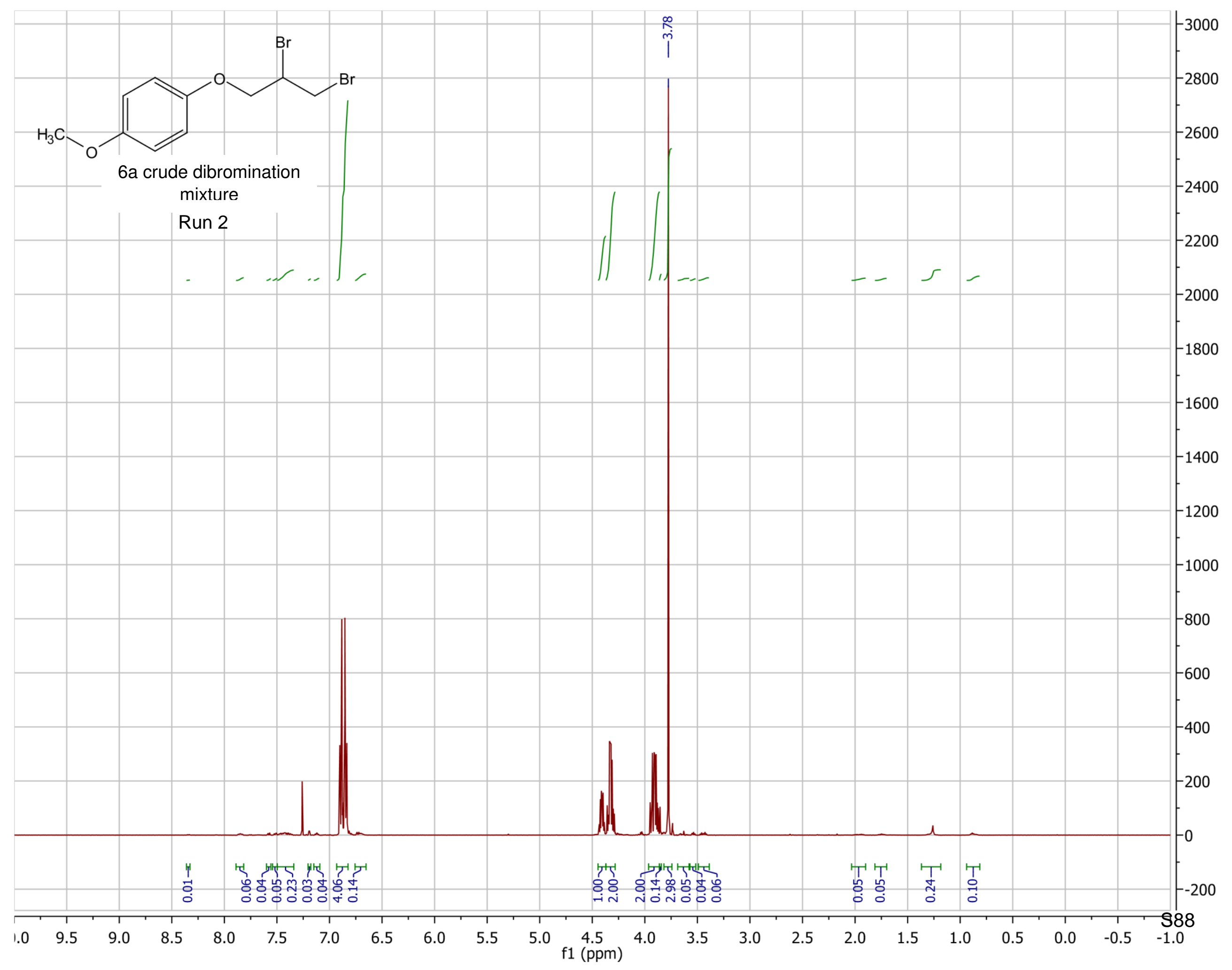




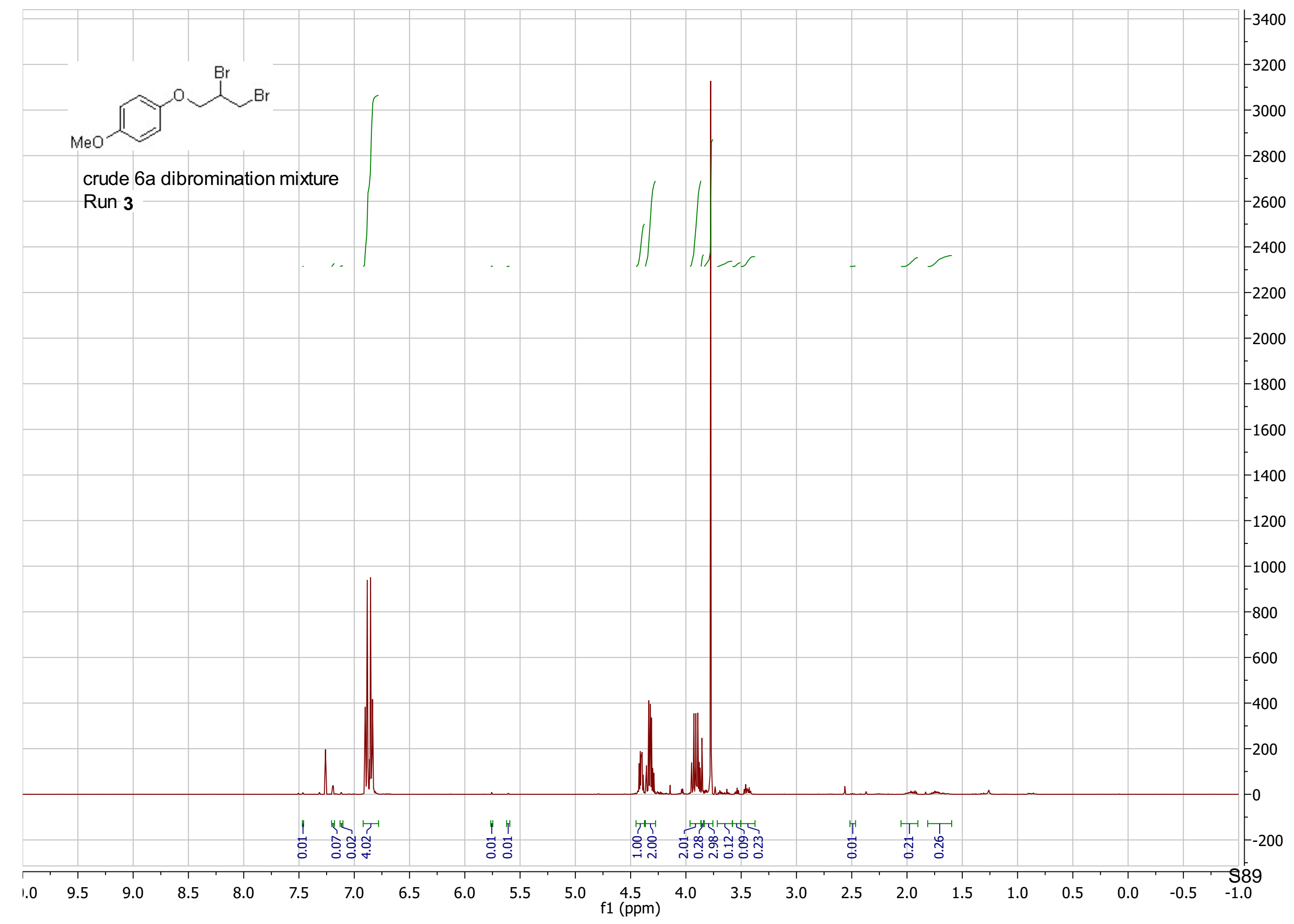




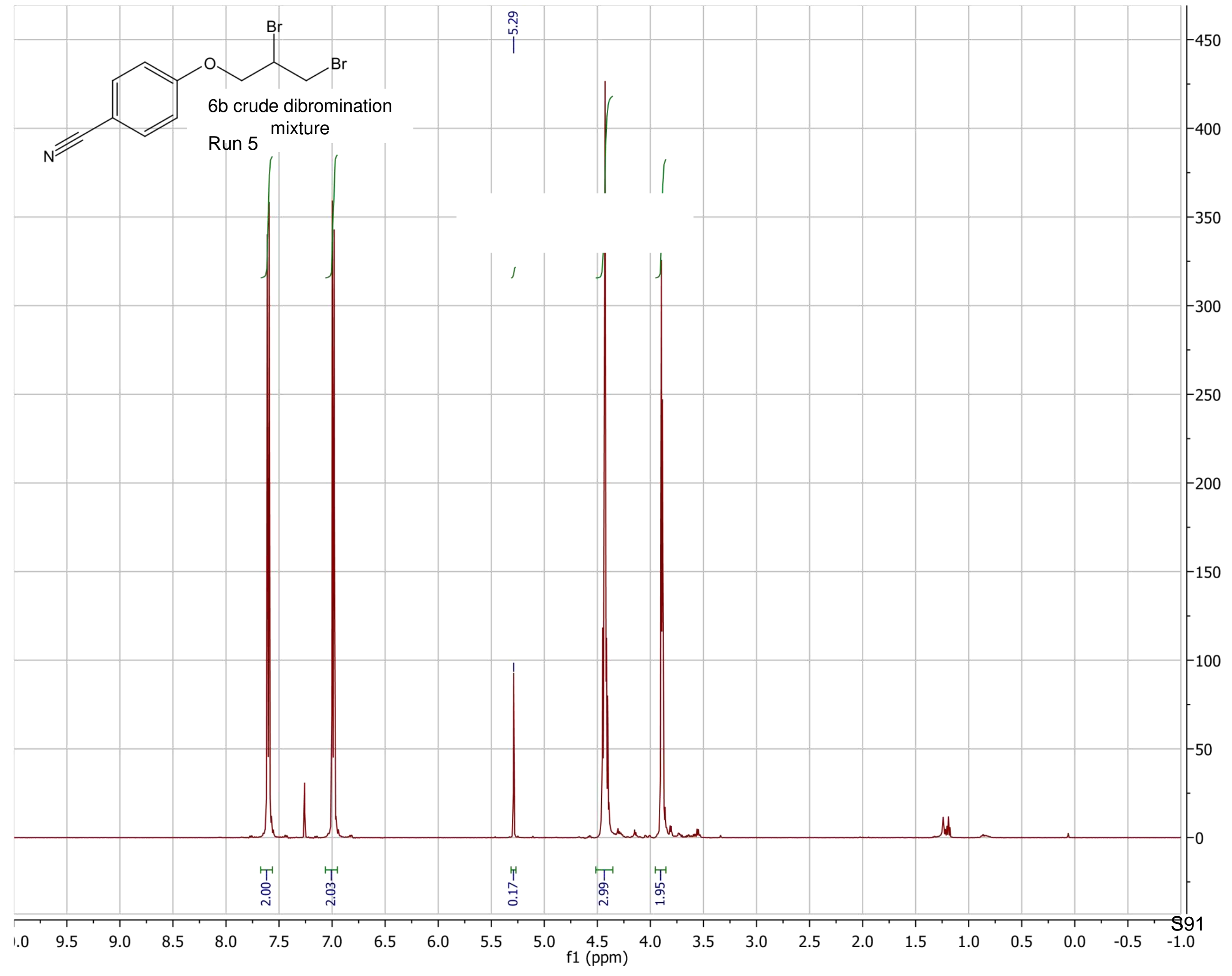




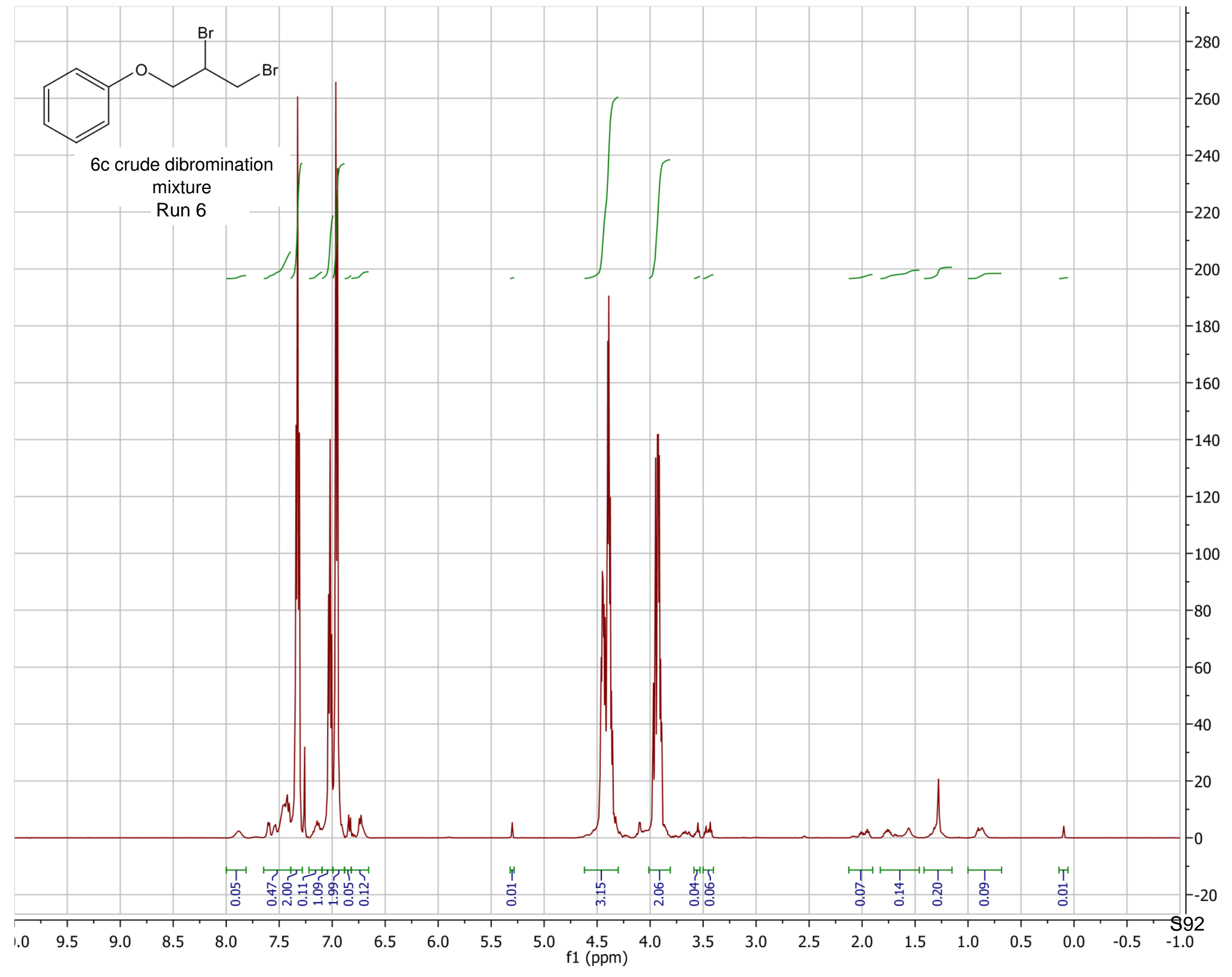




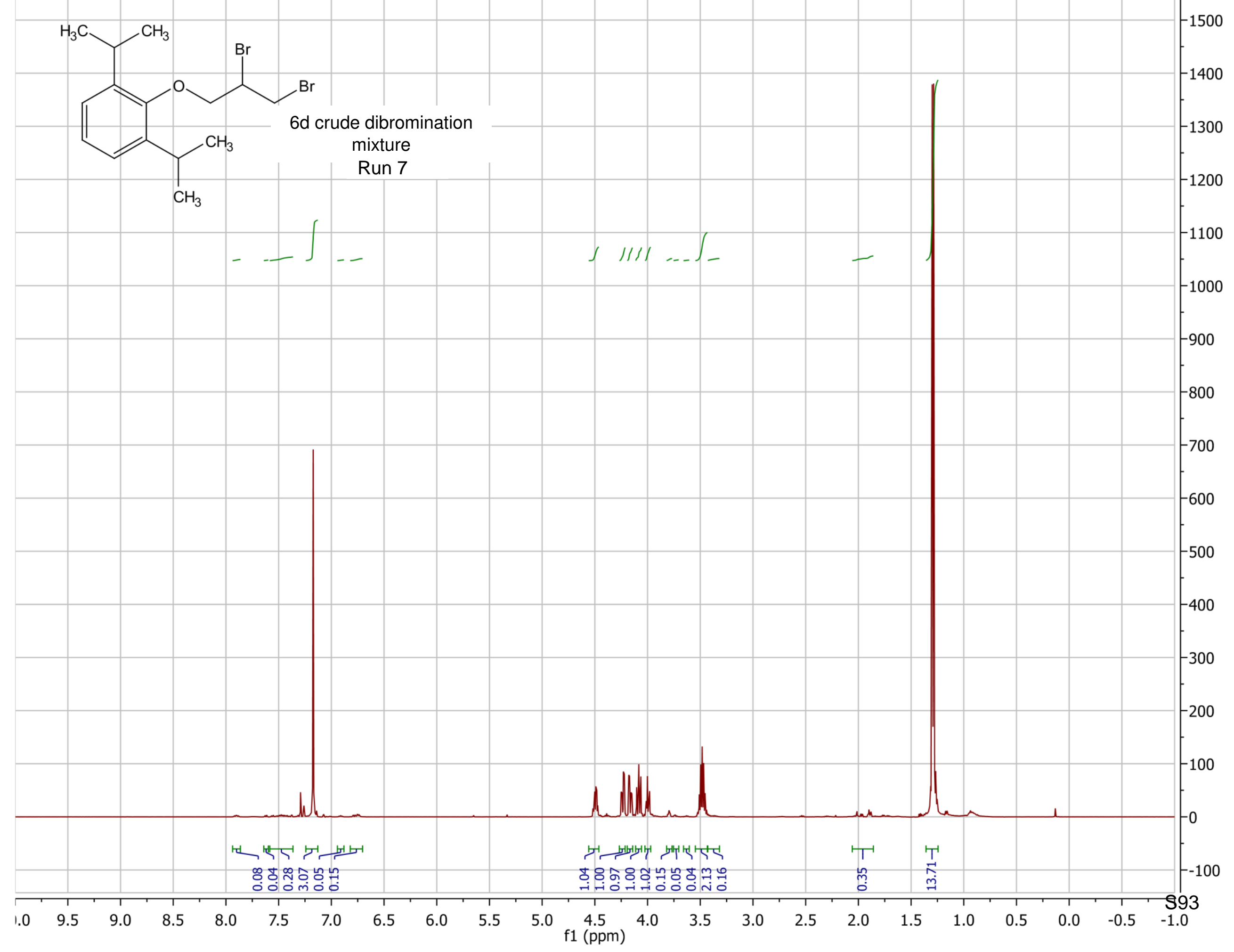




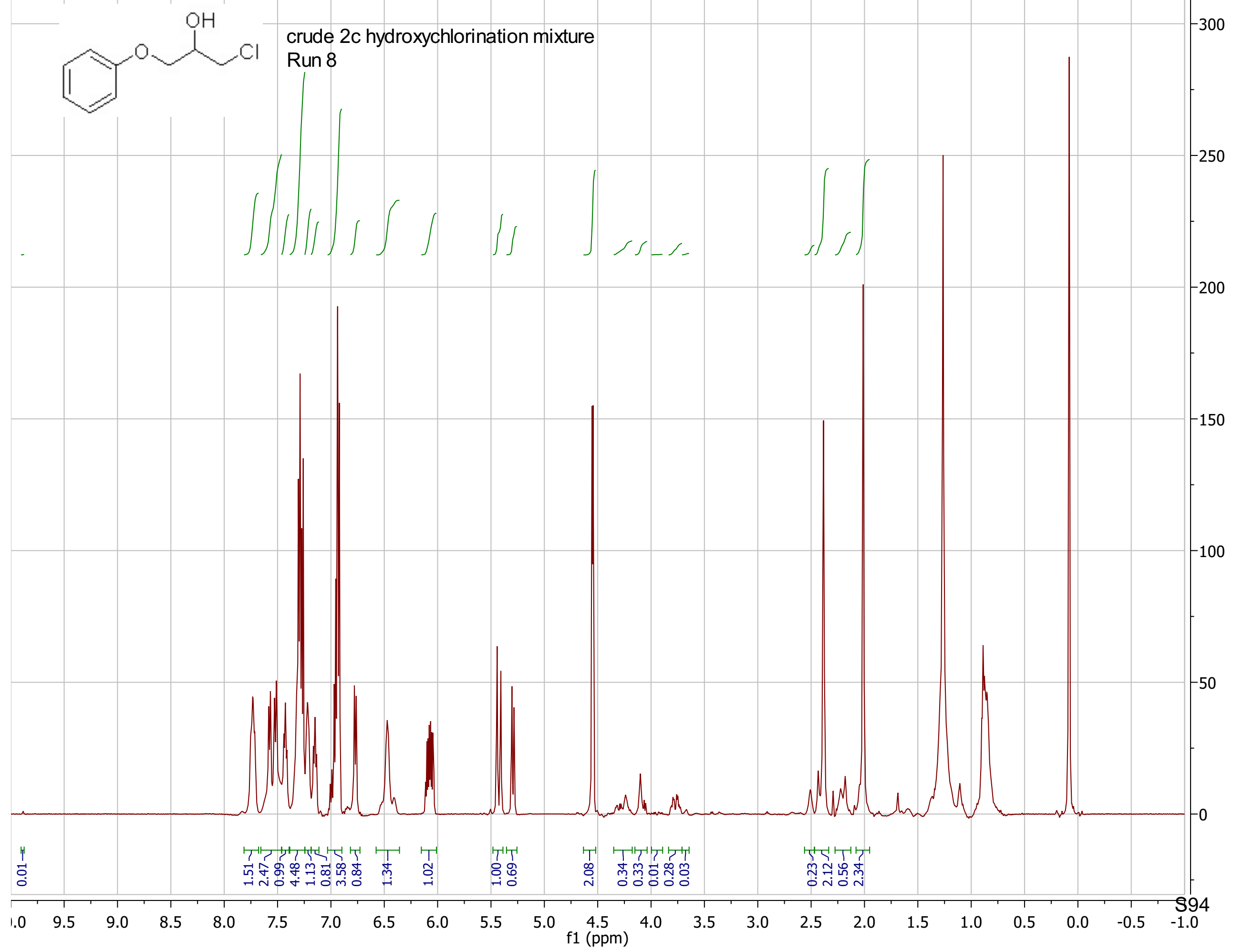




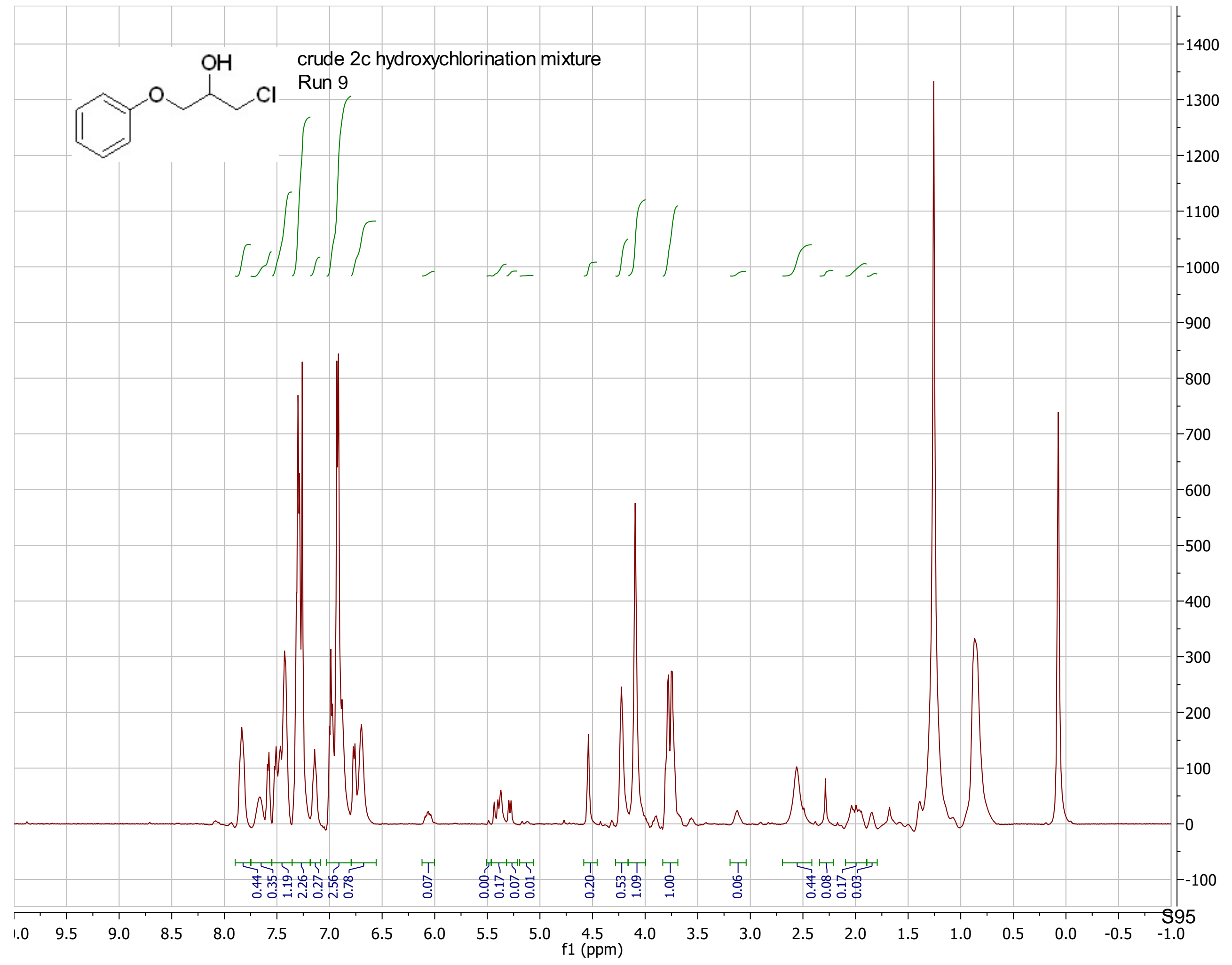




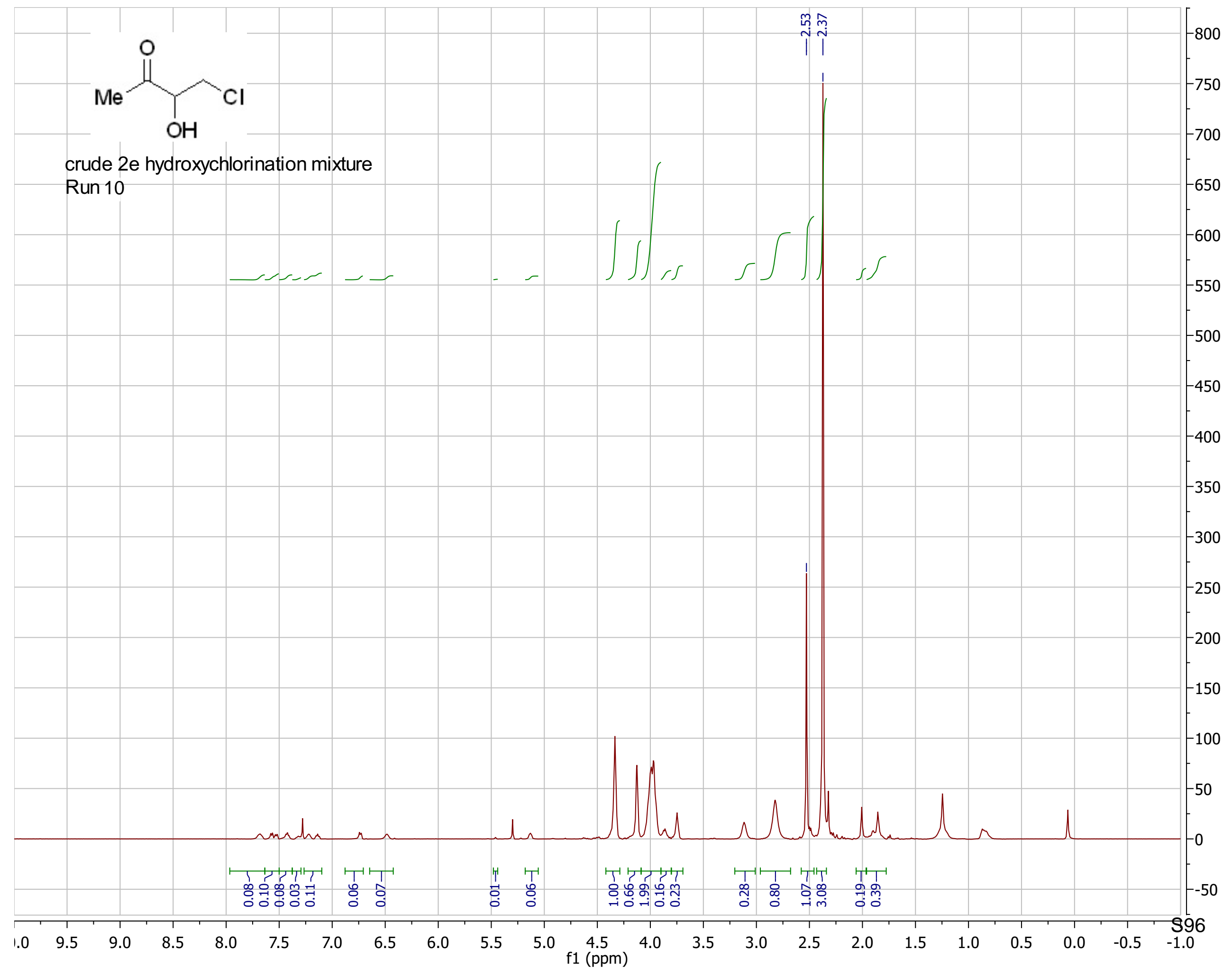




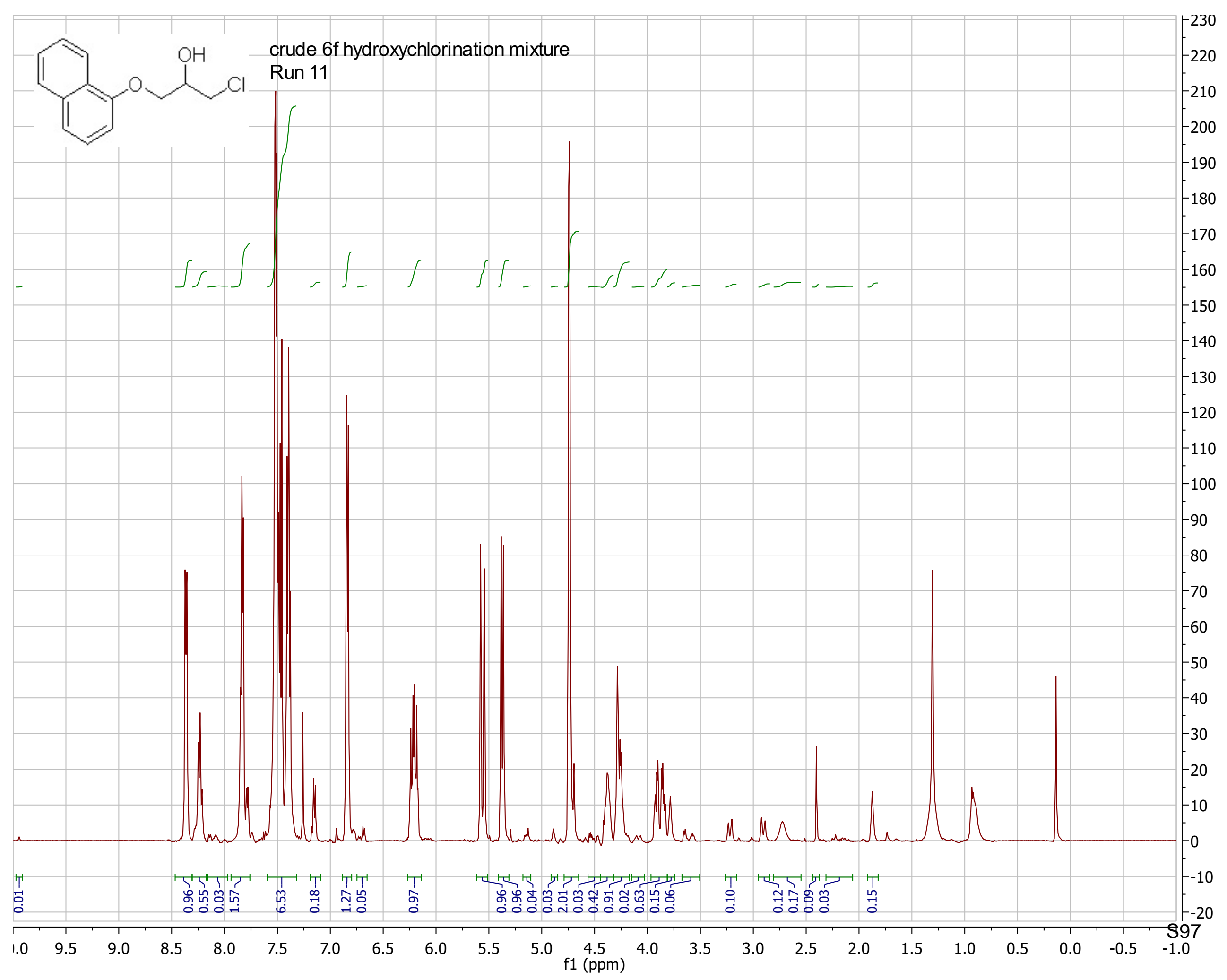

